\title{
The Role of Flavors in Electronic Cigarette Abuse Liability in Tobacco-Naïve Young Adults
}

Ilana Haliwa

West Virginia University, ih0010@mix.wvu.edu

Follow this and additional works at: https://researchrepository.wvu.edu/etd

Part of the Health Psychology Commons

\section{Recommended Citation}

Haliwa, Ilana, "The Role of Flavors in Electronic Cigarette Abuse Liability in Tobacco-Naïve Young Adults" (2020). Graduate Theses, Dissertations, and Problem Reports. 7663.

https://researchrepository.wvu.edu/etd/7663

This Thesis is protected by copyright and/or related rights. It has been brought to you by the The Research Repository @ WVU with permission from the rights-holder(s). You are free to use this Thesis in any way that is permitted by the copyright and related rights legislation that applies to your use. For other uses you must obtain permission from the rights-holder(s) directly, unless additional rights are indicated by a Creative Commons license in the record and/ or on the work itself. This Thesis has been accepted for inclusion in WVU Graduate Theses, Dissertations, and Problem Reports collection by an authorized administrator of The Research Repository @ WVU. For more information, please contact researchrepository@mail.wvu.edu. 
The Role of Flavors in Electronic Cigarette Abuse Liability in Tobacco-Naïve Young Adults

\author{
Ilana Haliwa
}

Thesis submitted to the Eberly College of Arts and Sciences at West Virginia University

In partial fulfillment of the requirements for the degree of

Master of Science in Psychology

Melissa Blank, Ph.D., Chair

Kevin Larkin, Ph.D.

Kris Martens, Ph.D.

Department of Psychology

West Virginia University

Morgantown, West Virginia

2018

Keywords: electronic cigarettes, young adults, abuse

Copyright 2020 Ilana Haliwa 


\begin{abstract}
The Role of Flavors in Electronic Cigarette Abuse Liability in Tobacco-Naïve Young Adults Ilana Haliwa
\end{abstract}

A primary public health concern associated with the use of electronic cigarettes (ECIGS) has been the risk of product initiation by vulnerable young adults who are largely naïve to tobacco. The use of ECIGs among such individuals may be influenced by the wide variety of sweet flavored liquids available for purchase. Previous work suggests that one flavor, menthol, increases the likelihood of abuse of cigarettes. Thus, the United States Food and Drug Administration has requested input from the research community regarding the abuse liability of flavored ECIGs in order to inform future product regulation. Using a double-blind, withinsubject study design, 30 young adults (aged 18-24 years, $M=19.43,63.3 \%$ female, $83.3 \%$ Caucasian) who are largely tobacco-naïve experienced two sessions that differed by the flavor of ECIG liquid used: tobacco or fruit (choice of fruit medley or mango). Within each session, participants used the flavor assigned ECIG during two separate puffing bouts and rated product effects via subjective questionnaires (e.g., nicotine delivery, product acceptability) pre- and postbouts. Participants also rated subjective product appeal and engaged in both a progressive ratio and probability-based purchase task to assess willingness to work for and spend money on each flavor of ECIG. Smoking topography (i.e., puff number, duration, and inter-puff interval) was also analyzed. Heart rate and subjective measures of nicotine delivery (e.g., lightheadedness, nausea, headache) were significantly higher post- bout compared to pre-bout, indicating nicotine delivery during puffing bouts. However, few significant effects of flavor emerged across subjective, physiological, behavioral economic, or puffing topography outcomes. Recruited participants had very low levels of lifetime ECIG use $(M=12.37$ uses; range $=3-50$ uses $)$ and 
reported not enjoying ECIG use. Rather, the majority of participants reported social motivations as a primary reason for product use. While this study is the first to experimentally assess abuse liability of flavor among a sample of tobacco naïve young adults, results suggest that there may be factors other than flavor which motivate initial product use and susceptibility among naïve users with low lifetime use. 
Table of Contents

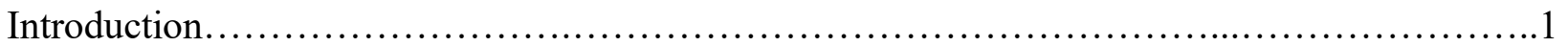

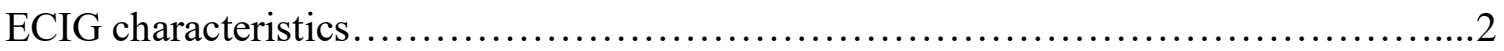

The History of Flavoring in Tobacco Products...................................... 3

Flavorings in ECIG Products................................................. 7

Statement of the Problem............................................................

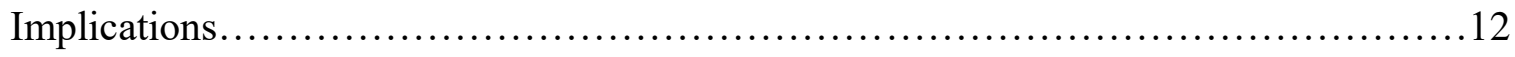

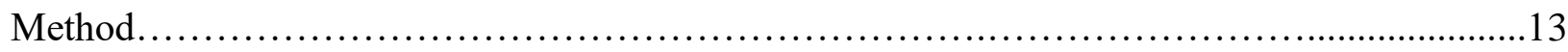

Selection of Participants...................................................... 13

Inclusion Criteria.......................................................... 13

Exclusion Criteria........................................................ 14

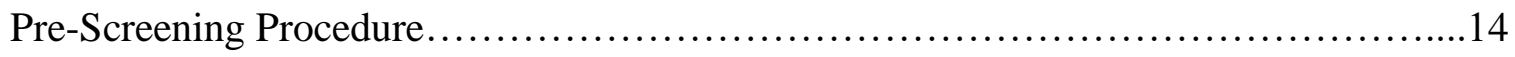

Informed Consent and In-person Screening Procedures...............................15

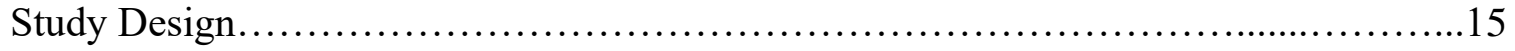

Session Procedure........................................................... 16

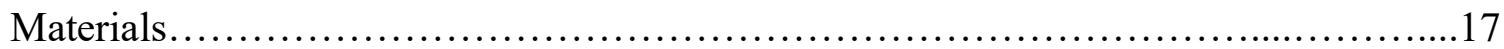

ECIG Devices and e-liquid............................................17

ECIG Topography Devices............................................17

Primary Outcome Measures................................................... 18

Direct Effects of Product Scale.............................................. 18

Progressive Ratio Task..................................................... 18

Probability Based Purchase Task.......................................... 18

Secondary Outcome Measures..................................................19

Direct Effects of Nicotine Scale.......................................... 19

Puff Topography.........................................................19

Physiological Measures..................................................20

Generalized Labeled Magnitude Scale ...................................20

ECIG Risk Perception.....................................................20

Electronic Cigarette Outcome Expectancy ...............................20

Flavor Discrimination Questionnaire ................................... 21

Exit Interview.....................................................21

Participant Safety and Rights...............................................21

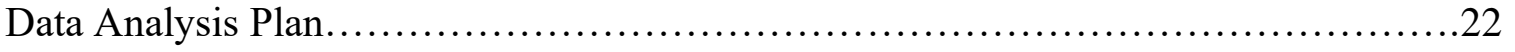

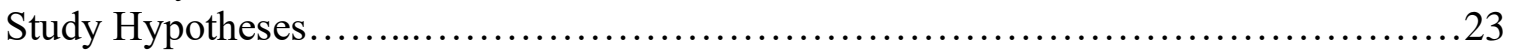

Results......................................................................... 24

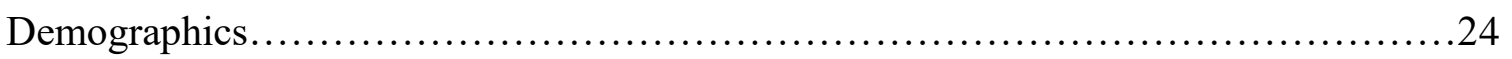

Primary Outcome Measures................................................... 24

Direct Effects of Product Scale...........................................24

Progressive Ratio Task..................................................27

Probability Based Purchase Task ..........................................27

Secondary Outcome Measures..................................................27

Direct Effects of Nicotine Scale.........................................27

Puff Duration............................................................ 30

Physiological Measures............................................... 31 
Generalized Labeled Magnitude Scale ....................................33

ECIG Risk Perception...................................................33

Electronic Cigarette Outcome Expectancy ..................................33

Flavor Discrimination Questionnaire...................................... 34

Exit Interview..........................................................

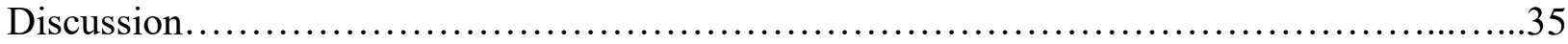

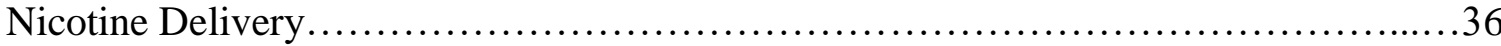

Subjective Ratings of ECIGs................................................ 37

Behavioral Economic Outcomes................................................ 38

Race Effects................................................................... 40

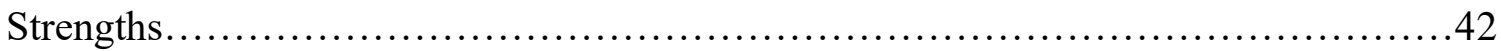

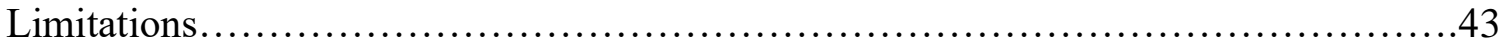

Conclusions and Future Directions........................................... 47

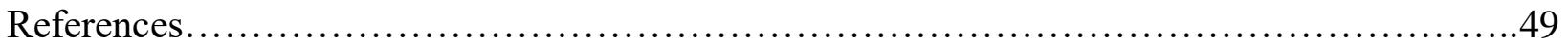

Tables

Table 1: Participant Baseline Characteristics...................................71

Table 2: Repeated Measures ANCOVAs by Flavor Condition........................72

Table 3: Mixed Repeated Measures ANOVAs by Race..............................73

Table 4: Repeated Measures ANOVAs by Order....................................74

Table 5: Repeated Measures ANOVAs for Behavioral Economics Outcomes............75

Table 6: Means and Standard Deviations for Behavioral Economics Outcome............76

Table 7: Means and Standard Deviations for ECIG Outcome Expectancies...............77

Table 8: Frequencies of ECIG Flavor Preference................................. 78

Table 9: Exit Interview.................................................... 79

Figures

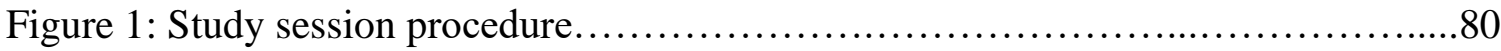

Figure 2: Mean "dizzy" ratings for bout x time x race effects.........................81

Figure 3: Mean "pleasant" ratings for flavor x bout x time $\mathrm{x}$ race effects ................82

Figure 4: Fruit Flavor Demand Curve for the Probabilistic Purchase Task ..............83

Figure 5: Tobacco Flavor Demand Curve for the Probabilistic Purchase Task ............84

Figure 6: Mean "confused" ratings for flavor $x$ bout $x$ time effects ....................85

Figure 7: Mean "nauseous" ratings for flavor $x$ bout $x$ race effects...................... 86

Figure 8: Mean "lightheaded" ratings for flavor x time $\mathrm{x}$ race effects..................87

Figure 9: Mean "headache" ratings for bout $x$ time $x$ race effects....................... 88

Figure 10: Mean diastolic blood pressure for flavor $\mathrm{x}$ bout $\mathrm{x}$ race effects...............89

Figure 11: Mean heart rate for bout $\mathrm{x}$ time $\mathrm{x}$ race effects............................ 90

Figure 12: Mean heart rate for flavor $\mathrm{x}$ bout $\mathrm{x}$ time $\mathrm{x}$ race effects .......................91

Figure 13: Mean systolic blood pressure for flavor $\mathrm{x}$ bout $\mathrm{x}$ time $\mathrm{x}$ race effects ..........92

Figure 14: Mean diastolic blood pressure for flavor $\mathrm{x}$ bout $\mathrm{x}$ time $\mathrm{x}$ race effects .........93

Figure 15: Mean heart rate for order $x$ bout $x$ time effects $\ldots \ldots \ldots \ldots \ldots \ldots \ldots \ldots \ldots \ldots . \ldots 9$ Appendices

Appendix A: Telephone Screening Questionnaire............................... 95

Appendix B: Demographic Information........................................ 98

Appendix C: Medical History and Drug Use Form................................ 99

Appendix D Electronic Cigarette Outcome Expectancy Short Scale ...................101

Appendix E: ECIG Risk Perception......................................... 102 


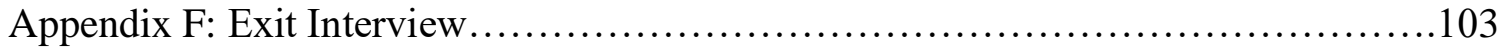

Appendix G: Direct Effects of Nicotine Scale ................................... 105

Appendix H: Direct Effects of Product Scale ........................................106

Appendix I: Generalized Labeled Magnitude Scale .................................. 107

Appendix J: Flavor Discrimination Questions................................... 110 
The Role of Flavors in Electronic Cigarette Abuse Liability in Tobacco-Naïve Young Adults As of June 22nd, 2009, the Food and Drug Association (FDA) has been granted authority to regulate tobacco products in the United States (U.S.) (H.R. 1256, 2009). Tobacco products subject to regulation under this law currently include cigarettes, cigarette tobacco, chewing tobacco, roll-your-own-tobacco, and smokeless tobacco. On August 8th, 2016, the FDA announced an extension of its regulatory authority to products such as hookah, pipe tobacco, cigars, and electronic cigarettes (ECIGs) (Food and Drug Administration Deeming Tobacco Products to be Subject to the Federal Food, Drug, and Cosmetic Act, 2016). These changes come on the heels of recent national survey findings, which demonstrate a jump in past month ECIG use among high school students from $1.5 \%$ in 2011 to $20.8 \%$ in 2018 (Cullen et al., 2018). Further, there was a 78\% increase in current ECIG use among high school students just between 2017 and 2018 (Cullen et al., 2018). In fact, data from the 2018 National Youth Tobacco Survey (NYTS) show that ECIGs are the most commonly used tobacco product among U.S. middle and high school students (Gentzke et al., 2019). ECIG use has also increased among young adults aged 18-24, with a 46.2\% increase in current use between 2017 and 2018 alone (Dai \& Leventhal, 2019). Among young-adult never-users of combustible cigarettes, a corresponding increase of $43.75 \%$ in current ECIG use was found (Dai \& Leventhal, 2019). Fortunately, current FDA regulations include prohibition of sales to minors, free samples to consumers, and vending machine sales, as well as the requirement of labels that warn of the addictive nature of nicotine (Food and Drug Administration Deeming Tobacco Products to be Subject to the Federal Food, Drug, and Cosmetic Act, 2016). Still, ECIGs remain available to young adult consumers, including those who are naïve to nicotine. The FDA has thus called for input from the scientific community regarding the regulation of these novel devices (FDA, 2017). 


\section{ECIG Characteristics}

"ECIG" is a term used to describe an electronic nicotine delivery system which uses a liquid that typically contains nicotine, propylene glycol, vegetable glycerin, and flavoring. These devices consist of a storage component (e.g., a tank or a cartridge) to house the liquid, as well as a battery, heating element (e.g., resistance wire coil), and flow sensor (Brown \& Cheng, 2014). The battery powers the heating element, which heats the liquid into an aerosol to be inhaled by the user (FDA, 2018). ECIG devices have been categorized by the research community into one of three generations (see Farsalinos \& Polosa, 2014 for pictures). First-generation ECIGS are called "cig-alikes" because they are similar in design to combustible cigarettes, with mouthpieces and LED lights mimicking the filter and burning end of a cigarette rod, respectively. They often come with pre-filled cartridges containing the liquid solution (Cassidy, 2011; Etter 2012). Second-generation ECIGs utilize refillable tanks or cartomizers to hold the liquid, and their batteries are typically capable of reaching higher voltages than first generation devices (Farsalinos et al., 2014). Finally, third-generation ECIGs (also known as "mods" or "variable voltage devices") allow the user more control over certain features. For instance, the battery may be replaced, and the voltage output can be increased or decreased to the users' desired setting (Wagener et al., 2017). Some of these devices, often called "pod-mods," feature refillable or non-refillable pods containing ECIG liquid, paired with a battery. These various device features, in combination with features of the liquid, determine the level of nicotine delivered to the user.

One device feature demonstrated to affect nicotine delivery is battery power, measured in volts (V). Higher battery power results in higher temperatures for aerosolizing the liquid solution (Trehy et al., 2011). Consequently, both cig-alikes (Talih et al., 2015) and tank models 
(Kosmider et al., 2014) with battery power of $5.2 \mathrm{~V}$ have been shown to yield higher levels of nicotine compared to those of 3.3V. This fact may also explain why second-generation devices, which typically include a higher-powered battery, deliver nicotine more effectively than firstgeneration devices (Farsalinos et al., 2014). Another ECIG feature that may influence nicotine yield is the ratio of the humectants, which are used in ECIG liquid for vapor production. The most commonly used humectants are propylene glycol (PG) and vegetable glycerin (VG), and liquids with a higher ratio of PG relative to VG have been shown to deliver higher levels of nicotine (Kosmider et al., 2014; Spindle \& Eissenberg 2018). Of course, a higher concentration of nicotine in the liquid is directly related to higher nicotine delivery (Hiler et al., 2017). Finally, preliminary evidence suggests that the flavor of the liquid used may affect nicotine delivery (St. Helen, 2018). This topic has been understudied relative to those ECIG characteristics described above; however, the impact of flavor on nicotine delivery has been shown for other tobacco products. The history behind the use of flavor in such products is described below, followed by the application of this knowledge to understanding similar effects in ECIGs.

\section{The History of Flavorings in Tobacco Products}

The tobacco industry has long used flavoring in existing tobacco products to increase their appeal and acceptability to consumers. For cigarettes, those flavored with menthol have been available since 1927 (Gardiner, 2003). Today, mentholated cigarettes represent $26 \%$ of the market share of all cigarettes sold in the U.S. (Federal Trade Commission, 2017), and recent national-level survey data suggest that $40 \%$ of current smokers use menthol cigarettes (Cohn et al., 2018). In addition to menthol, a variety of flavors are used in non-cigarette tobacco products. Smokeless tobacco products, for instance, have been sold in mint, wintergreen, champagne, and a variety of fruit flavors (cherry, apple, peach) (Kostygina \& Ling, 2016; Tobacco Institute, n.d.). 
Waterpipes (also known as hookah, shisha, or narghile; Maziak et al., 2015) are used with a sweetened tobacco called maassel, and the most popular choice of flavor is that of fruit (e.g., double apple, strawberry, mango) (Rose et al., 2018). Hookah and smokeless tobacco are among the most prevalent flavored products used by youth (aged 12-17 years), young adults (aged 18-24 years), and adults (25+). For those among these age groups who used these products in the past 30 days, prevalence of flavored product use was $62 \%, 57.2 \%$ and $48.2 \%$, respectively for flavored smokeless tobacco, and 71.5\%,68\%, and 62.2\%, respectively for flavored hookah (Rose et al., 2018). Cigars and cigarillos products, with flavors such as wine and vanilla, are also popular among users (Kostygina, Glantz, \& Ling, 2014). Between 2014 and 2015, prevalence of flavored cigarillo-use among youth (12-17), young adults (18-24), and adults (>25) who had used the product in the past 30 days was $52.3 \%, 51 \%$ and $51.1 \%$, respectively (Rose et al., 2018). Similarly, use of flavored cigars among young adults and adults who used the product in the past 30 days was $47.2 \%$ and 50.2\%, respectively (data not reported for youth aged 12-17; Rose et al., 2018). The proliferation of flavors available for these non-cigarette tobacco products has been controversial, as tobacco companies have capitalized on flavor properties to market directly to a previously untapped demographic - tobacco-naïve youth (Carpenter et al., 2009; Kostygina \& Ling, 2016).

Marketing flavored tobacco products to tobacco-naïve youth serves the ultimate purpose of attracting a new generation of tobacco users. Flavoring can promote tobacco use through one of several different mechanisms, such as reduction of the initial aversive effects of tobacco use, serving as a reinforcing sensory cue when repeatedly paired with nicotine, and increasing the bioavailability of nicotine (Wickham, 2015). As for the first mechanism, the addition of flavoring is thought to improve taste by masking the harshness and bitterness of tobacco 
(Carpenter et al., 2005; Cummings et al., 2002). Some flavors, such as menthol, may also soothe irritated airways during inhalation (Kreslake et al., 2010; Wickham, 2015; Wickham et al., 2017). In line with this idea, among individuals who initiated tobacco use in the past 30 days, $70 \%$ of youth (aged $12-17$ years), $55 \%$ of young adults (aged 18-24 years), and $40 \%$ of adults (aged > 25) did so with a flavored product (Rose et al., 2018). Youth and young adults have also reliably rated flavored tobacco products more appealing than unflavored products (Ambrose et al., 2015; Krishnan-Sarin et al., 2015; McDonald \& Ling, 2015). The second mechanism through which flavors may promote tobacco use is that of conditioning, with the flavor becoming associated with nicotine reinforcement as the two are repeatedly administered together (Wickham, 2015). Secondary reinforcers are particularly powerful in maintaining smoking behavior, as nicotine has shown to act as a reinforcement enhancer when paired with external cues (Chaudhri et al., 2006). For instance, smokers of mentholated cigarettes rate smoking as less rewarding when the menthol cue is removed (Rose \& Behm, 2004). These same smokers were observed to be more resistant to extinction of reward responses to mentholated cigarettes than non-mentholated cigarette smokers were to their usual non-mentholated cigarettes (Rose \& Behm, 2004). The third mechanism involves the influence of menthol on the bioavailability of nicotine in tobacco products. Specifically, menthol decreases elimination of nicotine in the body through inhibition of metabolic enzymes, thereby increasing the amount of nicotine in the bloodstream relative to smoking cigarettes without menthol (Benowitz, Herrera \& Jacob, 2004; Perez-Stable \& Benowitz, 2011). These and other potential mechanisms are described in detail elsewhere (Wickham, 2015).

Notably, the tobacco industry has been aware of these mechanisms for years and has used this understanding to facilitate product initiation (Kostygina \& Ling, 2016). Internal company 
documents outline a "graduation strategy," for example, for those naïve to smokeless tobacco (Connolly, 1995). Such individuals were deemed likely to begin use of smokeless tobacco with products relatively low in nicotine concentration but high in flavor content (U.S. Smokeless Tobacco Company, 1987). Indeed, “starter” smokeless tobacco products were developed to encourage initiation of smokeless tobacco use (Carpenter et al., 2009), with users expected to graduate from milder to more full-bodied products with relatively high nicotine concentration and less flavoring (Kostygina \& Ling, 2016; U.S Smokeless Tobacco Company, 1987). Other companies used similar strategies for cigarettes, perhaps based on internal studies conducted with consumers. These studies found that cigarettes with sweet flavors were more appealing to tobacco users who were younger and less experienced (Brown \& Williamson, 1984; Philip Morris, 1992; R.J. Reynolds, 1988). Subsequent years saw an increase in the number of brands that were sold in a variety of flavors, including KOOL Midnight Berry, Camel Twista Lime, and Camel Mandarin Mint (Carpenter et al., 2005). Today, all flavors (except for menthol) in cigarettes are prohibited by the 2009 Family Smoking and Tobacco Prevention Act. Flavored ECIGs are in the early stages of regulation by the FDA. As such, no formal regulations have been implemented at the national level. At the state level, only New York, Massachusetts, Rhode Island, and Washington have enacted legislation regulating the sale of ECIGs (National Conference of State Legislatures, 2019). While all four states prohibit the sale of flavored nicotine and/or vaping products, Massachusetts has implemented the strictest legislation yet, banning the sale of any vaping products regardless of flavor (National Conference of State Legislatures, 2019). 


\section{Flavorings in ECIG Products}

In the majority of U.S. states, ECIG liquids are available in many flavor varieties, with one report demonstrating the existence of over 7700 different options: fruit (e.g., strawberry, mango, banana), dessert (e.g., banana split, caramel, chocolate), candy (e.g., cotton candy, skittles, gummy worms), alcohol/drinks (e.g., red bull, mojito, piña colada) flavors, among others (Zhu et al., 2014). Those flavors that simulate fruit tastes are most popular. In a review of ECIG flavor-related content via a web-based social networking forum (Reddit), researchers found that fruit flavors $(n=15,720)$ were mentioned most frequently followed by cream $(n=10,289)$, tobacco $(n=7,475)$, menthol $(n=3,421)$, and others (n's $\leq 3,347)$ (Wang et al., 2015). Of those posts that mentioned fruit flavors specifically, strawberry was the most popular (3,657 posts), with almost twice as many references as the second most popular flavor, banana (1,864 posts) (Wang et al., 2015).

Among the most common reasons for ECIG use among youth and young adults is the fact that they are available in a variety of appealing flavors (Tsai et al., 2018; Villanti et al., 2017). Nearly $61 \%$ of young adults aged 18-24 who have tried ECIGS report that their first use occurred with a flavored liquid (Villanti et al., 2017). Similar results emerged from the 2014-2015 Population Assessment of Tobacco and Health Survey in which 81.6\% of youth aged 12-17 and $74 \%$ of young adults aged 18-24 who initiated ECIG use in the past year, reported doing so use with a flavored product (Rose et al., 2018). Additionally, fruit flavors are more likely to be tried by never-smoking adolescents than by current smokers attempting to quit (Ford et al., 2016). The appeal of flavors to younger individuals is further supported by experimental-based work; relative to adult smokers aged $\geq 25$ years, those aged 18 -24 years were more likely to make hypothetical purchases of ECIGs when they were available in flavors (Pesko et al., 2016). These 
and other findings suggest that "kid appealing flavors" (FDA, 2017) may be influencing the initiation and/or maintenance of ECIG use among certain populations. This idea has been examined systematically in only a few studies, as described below.

In one study (Walele et al., 2016), the nicotine delivery of a cig-alike ECIG product with and without menthol flavor (as well as an own brand of cigarette and a nicotine inhaler; results not discussed here) was examined in a sample of adult (mean age $=31.1$ years) daily smokers with no previous ECIG experience. Within each condition, participants used their assigned product once per hour for four consecutive hours. Each puffing bout was standardized such that participants took 10 puffs every 30 seconds, and each puff was of 4-second duration. Blood samples were taken before and after each bout. No significant differences were observed between ECIG flavors in terms of nicotine absorption or elimination rates. A potential limitation of this study, however, is that the ECIG device used delivered negligible levels of nicotine to the user relative to a cigarette (i.e., maximum concentration of 2.5-3.6 ng/ml for the ECIG flavors vs. $21.2 \mathrm{ng} / \mathrm{ml}$ for the cigarette). Consequently, the range of possible plasma nicotine levels observed after ECIG use was restricted.

Other work was designed to compare nicotine intake between ECIG flavors of tobacco, strawberry, and participants' usual flavor (St. Helen et al., 2017; St. Helen et al., 2018). This within-subject study included 14 adult (mean age $=32.3$ years) users of ECIGs, either exclusively ( $\mathrm{n}=4$ never smokers; $\mathrm{n}=12$ former smokers) or concurrently with cigarettes $(<5$ cigarettes per day). Within each condition, participants engaged in a single 15-puff directed bout in addition to a 90-minute ad libitum bout. Relative to tobacco flavor, strawberry flavor resulted in significantly longer puffs from the ECIG, a higher (non-significant) maximum concentration of nicotine in plasma, and comparable subjective ratings of liking and enjoyment (St. Helen et 
al., 2017; 2018). Also observed was that, relative to both strawberry and tobacco flavors, participants' usual flavor resulted in significantly more and longer puffs from the ECIG, and significantly higher ratings of items that denote product acceptability (St. Helen et al., 2017; 2018). The lack of a significant difference between flavors for the subjective ratings of product acceptability may be influenced by the participants' previous ECIG use. That is, these experienced ECIG users (mean = 2.3 years of ECIG use) reported that their preferred flavors were largely those other than fruit: cream/honey/milk $(n=3)$, drink $(n=3)$, and more than one flavor $(n=5)$ versus fruit/candy $(n=3)$.

In other experimental work, however, participants' subjective experience of use has differed as a function of ECIG liquid flavor. One study included 31 adult (mean age $=33.6$ years) ECIG users, who either used ECIGS exclusively (daily use of a medium nicotine strength liquid for at least the past month) or dual users of ECIGS and cigarettes (daily cigarette smoking for at least the past year and ECIG use at least 3 days/week) (Kim et al., 2016). Participants were instructed to take 4 puffs from each of 6 commercially available flavors using a secondgeneration device, and rated various subjective effects following each bout. All flavors were used within a single session, and a "palate cleansing" procedure was administered in between each use. Ratings of liking were correlated positively with ratings of sweetness and coolness (r's $=0.31 \& 0.25$, p's $<.001)$ and correlated negatively with ratings of bitterness and harshness (r's $=-0.25 \&-0.29$, p's $<.001)$. The tobacco flavored liquid was perceived as the least sweet and the least liked. Similar relationships between flavor properties and product appeal have been demonstrated in other studies of adult ECIG users. For instance, 20 adult (19-34 years) ECIG users (weekly ECIG use > 1 month) who smoked less than 15 cigarettes per day were recruited to self-administer 20 different ECIG solutions (10 flavors each at 0 and $6 \mathrm{mg} / \mathrm{ml}$ doses) from a 
second-generation device (Goldenson et al., 2016). Sweet flavors resulted in significantly higher ratings of liking and willingness to use the product again, compared to non-sweet flavors. Participants also stated that they would be willing to pay a higher amount of money for a day's worth of sweet flavored liquid than non-sweet flavored liquid (Goldenson et al., 2016). Together, these studies provide initial laboratory evidence for the role of flavors in ECIGS on product appeal among ECIG users.

In addition to using self-report measures, some researchers have made use of behavioral economics tasks to assess abuse liability of various ECIG flavors. The field of behavioral economics represents a fusion of psychology and economics, in which behavioral principles can be used to predict economic outcomes (e.g., willingness to purchase a particular item, the effects of a tax increase on purchasing behavior, the effects of a delay until the item is received). In a study of 32 daily cigarette smokers aged $18-30$ (mean age $=25.0$ years), with an average of 12.0 (15.4) lifetime ECIG uses, participants self-administered three ECIG flavors (green apple, chocolate, and unflavored) from a second-generation device (Audrain-McGovern, Strasser \& Wileyto, 2016). They then rated each flavor based on its subjective reward value and engaged in 90-minute ad libitum bout with the option to use any flavor of their choice. Participants also completed a behavioral economics task, whereby they used a computer mouse to hit targets on one of two computer screens to earn points towards puffs from either a flavored or unflavored ECIG, with the option to switch between computers at will. Reinforcement for the unflavored ECIG was delivered on a fixed ratio schedule, with 25 target hits required to earn a point. Reinforcement for the flavored ECIG was delivered on a progressive ratio schedule, such that each successive point required 25 more target hits than the previous one. Willingness to put forth increasing levels of effort for access of a flavored ECIG serves as a measure of the reinforcement 
value of flavored relative to unflavored ECIGs. Participants rated both the green apple and chocolate flavored ECIG as more rewarding and were willing to put forth significantly more effort to obtain these flavors (i.e., perform more target hits), relative to the unflavored ECIG. Additionally, participants took twice as many puffs of the flavored liquid (40.29 puffs) as the unflavored liquid (23.22 puffs) during ad libitum use. Thus, in regular cigarette smokers with little ECIG experience, these sweet flavors were preferred over unflavored liquid as evidenced by both subjective and behavioral outcomes.

Behavioral economics tasks were also used to characterize the abuse liability and preference for flavored ECIG products in a two-part study of 36 adult (mean age $=37.8$ years) daily cigarette smokers with limited ECIG use (i.e., $\geq 1$ weeks for $\geq 1$ month) (Barnes et al., 2017). Participants were exposed to a second-generation ECIG flavored with either tobacco or menthol liquid (Study 1; $n=17$ ), or cherry or unflavored liquid (Study 2; $n=19$ ). They then engaged in tasks that measure the participants' willingness to purchase products across various price points. No significant differences were observed for willingness to purchase tobacco vs. menthol liquid; however, participants were willing to pay significantly more for the cherry flavored liquid than the unflavored liquid. That no differences were observed between tobacco and menthol may be due to the fact that the majority of participants were smokers of menthol cigarettes, as the menthol flavor profile of ECIG liquid may not be comparable to that for cigarettes.

Together, this work suggests that flavorings in liquid may contribute to the abuse liability of ECIG devices, at least among individuals who have a history of tobacco use. Little similar work exists in a population of vulnerable tobacco-naïve individuals. Thus, it remains unknown 
whether flavors have the potential to promote the use of ECIGs among individuals who are not tolerant to the effects of nicotine and who have had little exposure to tobacco-related cues.

\section{Statement of the Problem}

Use of ECIGS by youth and young adults has skyrocketed in recent years, with a $16 \%$ prevalence of past 30-day use in middle and high school students in 2015 , compared to $1.5 \%$ in 2011 (USDHHS, 2016). In 2014, the prevalence of lifetime ECIG use and past 30-day use in young adults $18-24$ years was $35.8 \%$ and $13.6 \%$, respectively. These rates are significantly higher for young adults than for adults 25 years and older (16.4\% and 5.7\%, respectively; USDHHS, 2016). Youth and young adults prefer flavored ECIG products to unflavored products (Ambrose et al., 2015; Krishnan-Sarin et al., 2015; McDonald \& Ling, 2015). In fact, among young adults who first tried within the past year, 74\% did so with a flavored product (Rose et al., 2018). Sale of flavored liquids may thus contribute to a continued rise in the prevalence of ECIG use, which has been shown to serve as a gateway for the use of combustible tobacco products (Leventhal et al., 2015; Primack et al., 2015; Wills et al., 2016). Initiation and subsequent "graduation" of non-tobacco users from experimentation with flavored nicotine products to regular use has been a documented market strategy by the tobacco industry (David Weiss Associates, 1987). Laboratory research is needed to evaluate the role of flavors in likelihood of product initiation and abuse liability in tobacco-naïve young adults.

\section{Implications}

In light of the aforementioned findings regarding risk for smoking initiation in nonsmoking youth, the proposed study has implications ranging from smoking prevention to national policy regulation. The FDA has appealed to the scientific community to provide data on which to base future policy and regulation of ECIG products, with a particular emphasis on 
addressing the possibility of flavored ECIGS serving as a gateway product to regular tobacco/nicotine use. While abundant survey data highlights the role of flavor in the initiation of ECIG use by young adults, laboratory research such as this is needed to better characterize smoking behavior, reward value of flavored ECIG products, and the ability of flavor to mask the harshness of nicotine in this population.

\section{Method}

\section{Selection of Participants}

A total of thirty largely tobacco-naive individuals completed this within-subject study. This sample size was calculated using $\mathrm{G}^{*}$ power based on the power needed to detect the effects of primary interest (i.e., differences between flavors for subjective ratings of product acceptability and behavioral response via the behavioral economic tasks), assuming a small to medium effect size (Cohen's $\mathrm{f}=0.15-.20$; Cohen, 1992), a moderate correlation among repeated measures $(r=0.50)$, a Type I error rate of 0.05 , and a desired power of 0.80 .

\section{Inclusion criteria}

Healthy volunteers between the ages of 18 to 24 were recruited via word-of-mouth, and advertisements (e.g., flyers, electronic announcements) approved by the Institutional Review Board. To ensure that participants were largely tobacco-naïve, they were required to report fewer than 100 lifetime uses of any tobacco products (as in Bondy et al., 2009; CDC, 2017; Jones, Gulbis \& Baker, 2010; Ozga et al., 2016), as well as no tobacco use (excluding ECIGs) in the past 30 days. For use of ECIGs specifically, they were required to report at least 3 lifetime uses and to never have used an ECIG containing nicotine on 7 consecutive days. Participant recruitment was stratified in order to include relatively equal amounts of naïve users with low ECIG use ( $\leq 10$ lifetime uses) and those with relatively higher ECIG use ( $>10$ lifetime uses). 
These criteria were chosen with the goal of enrolling participants who were relatively inexperienced but not entirely naïve to ECIGs.

\section{Exclusion criteria}

Individuals were excluded if they reported any uncontrolled medical or psychiatric conditions, use of any other illicit drug use in the past month, or current breast-feeding or pregnancy (confirmed via urinalysis). Also excluded were volunteers reporting use of marijuana on more than five days of the past month. Based on similar route of administration, regular use of marijuana could affect ECIG puff topography outcomes (Agrawal \& Lynskey, 2008; Aung, Pickworth \& Moolchan, 2004; Fielder, Carey \& Carey, 2013). However, given the prevalence of marijuana use among young adults aged 18-24 (Lipari \& Jean-Francois, 2016; NIDA, 2017), we chose to limit past marijuana use rather than excluding any use. In order to protect the privacy of individuals divulging use of an illegal substance, a Certificate of Confidentiality was obtained from the National Institute of Drug Abuse (https://humansubjects.nih.gov/coc/background). Similar exclusion criteria have been successfully implemented in past studies (Blank et al., 2009; Breland et al., 2002; Cobb et al., 2010; Eissenberg et al., 1996; Ozga et al., 2016; Spindle et al., 2015).

\section{Pre-screening procedure}

Potential participants who responded to study advertisements first completed a prescreening interview (Appendix A) in which they responded to questions regarding basic demographic and medical history information, as well as past and current substance use, including nicotine, alcohol, and illicit substance use. Volunteers who were eligible based on the pre-screen were then asked to visit the laboratory for a detailed in-person screening. 


\section{Informed consent \& in-person screening procedures.}

Individuals were guided through the informed consent form to convey the study purpose, study procedures, and the potential risks and benefits of participation. Those who were able and willing to consent completed additional screening questionnaires assessing information similar to that obtained during the telephone screening (Appendix B \& C). This overlap in questionnaire items is intentional and allowed the researchers to ensure reliable reporting. Individuals whose responses were unreliable were excluded from further study participation. Nonsmoking status was confirmed with an expired air CO level of $\leq 5 \mathrm{ppm}$ (SRNT, 2002), measured via breath sampling with a non-invasive monitor (piCO+ Smokerlyzer. coVita; Haddonfield, NJ), as well as with a urinary cotinine value $<100$ ng/mL (NicAlert Nicotine Test. Jant Pharmacal Corporation; Encino, CA). Females were asked to provide a urine sample to allow for semi-quantitate testing of pregnancy. Finally, all participants were familiarized with the testing environment, questionnaires, and measurement equipment.

\section{Study Design}

This study used a within-subject design, with participants experiencing two conditions in random order that differ by the flavor of ECIG liquid administered: tobacco or fruit (choice between mango and fruit medley). Within each condition, participants used the flavor assigned ECIG during two separate puffing bouts, rated product effects via subjective questionnaires (e.g., nicotine delivery, product acceptability) pre- and post-bouts, and completed behavioral choice tasks (e.g., hypothetical purchase, progressive ratio). Following completion of the second session, participants completed a questionnaire assessing expectancies regarding the effects of ECIGs as well as the perceived risks of ECIGs relative to cigarette products (Appendices D \& E), as well as a semi-structured exit interview (Appendix F). 


\section{Session procedure}

All study sessions took place on the Downtown Campus of West Virginia University. Sessions were separated by a minimum of 48 hours to avoid carryover effects (as in Breland et al., 2002; Eissenberg et al.,1996; Lechner et al., 2015; Spindle et al., 2015), and were preceded by 12 or more hours of nicotine/tobacco abstinence. As shown in Figure 1, expired air CO level was measured at the beginning of each session in order to verify abstinence $(\leq 5 \mathrm{ppm})$ from combustible products such tobacco and marijuana (Blank et al., 2009; Spindle et al., 2015a; Tackett et al., 2015). Participants were then connected to physiological equipment for continuous measurement of heart rate and blood pressure. Thirty minutes later, participants completed baseline questionnaires: Direct Effects of Nicotine Scale (DENS, Appendix G; Evans et al., 2006) and Direct Effects of Product Scale (DEPS, Appendix H; Spindle et al., 2015a). Next, participants completed their first puffing bout, whereby they took one ECIG puff every 30 seconds for a total of 10 ECIG puffs as guided by laboratory staff (as in Spindle et al., 2015a; Vansickel et al., 2010; Vansickel \& Eissenberg, 2013). Directly after this bout, participants completed the DENS and the DEPS again. Thirty minutes after the first bout, participants experienced a second bout, identical to the first (subjective questionnaires, puffing bout, and subjective questionnaires). In between the two puffing bouts, participants completed a probabilistic purchase task. Forty-minutes after the end of the second puffing bout, participants engaged in a progressive ratio task. Following completion of the second study session, participants completed questionnaires assessing their understanding of and beliefs about ECIG products. Participants were compensated $\$ 25$ per session, for a total of $\$ 50$ for complying with all study requirements. 


\section{Materials}

ECIG devices and liquid. Participants used a JUUL ECIG (JUUL Labs; San Francisco, California). This brand of EC device has quickly become one of the most popular devices on the market and has been shown to be particularly appealing to young adults (Huang, Duan, Kwok, Binns,...Emery, 2018; Kavuluru, Han, and Hahn, 2018). Liquid for these devices come prepackaged in pods with $59 \mathrm{mg} / \mathrm{ml}$ nicotine and a ratio of 30:60 propylene glycol to vegetable glycerin. Liquid was either tobacco or fruit flavored and participants were given a choice between fruit medley and mango for the fruit condition. These flavors were chosen based on a combination of survey reports (Harrell et al., 2016; Rose et al., 2018; Shang et al., 2018), screening data from our ongoing laboratory work, as well as discussion with local retailers. Together, these sources suggest that fruit blends are a preferred flavor among ECIG users, including those who live within the greater Morgantown area. These sources also suggest that mango is a popular flavor, and thus would be an alternative choice for those who find the fruit blend flavor unappealing. Importantly, recently presented data demonstrate that JUUL delivers physiologically active doses of nicotine (Maloney et al., 2019b).

ECIG topography video scoring. Puff topography was measured via video recording (Vixia HF R700, Canon, Melville, NY). Puff onset was identified as the first video frame in which a user's lips were enclosed around the ECIG mouthpiece, while puff offset was defined as the last frame in which a user's lips were enclosed around the ECIG mouthpiece. Puff number was measured as the total number of discrete puffs (> 300 milliseconds) completed within a puffing bout. Puff duration was measured as the amount of time, in seconds, between the onset and offset of a single puff. Inter-puff interval also was measured in seconds and is the amount of times between the offset of one puff and the onset of a subsequent puff. All video-recorded data 
were scored by two independent raters and then compared for reliability using a minimum cutoff of ICC $\geq 0.90$. Latency to puff for the PRT was recorded as the time between a participant earning a reward and puff onset. The participant's screen is shown in the video, and thus scorers are able to see the exact time point at which a puff was earned. This method of topography measurement and scoring has been used successfully in prior work for cigarettes (Blank et al., 2009; Frederiksen et al., 1977; Lichtenstein \& Antonuccio, 1981) and for ECIGs (Felicione, 2016).

\section{Primary Outcome Measures}

Direct Effects of Product Scale. The Direct Effects of Product Scale (Appendix H; Blank et al., 2011; Spindle et al., 2015a; Vansickel et al., 2010) contains nine VAS items assessing effects commonly reported with ECIG smoking (e.g. "Was the ECIG satisfying?" and “Did the ECIG help you concentrate?"). Scale ratings range from zero to 100, with each item scored separately.

Progressive Ratio Task (PRT). A computerized progressive ratio task was used to assess ECIG self-administration (as in Copp et al, 2015; Barrett, 2010). Initially, ten key presses were required in order to earn the first puff of the ECIG. Subsequently, the number of key presses required to earn a puff increased by $30 \%$ after each puff. The outcomes for this task consist of break point (the maximum number of key presses to earn a puff), the number of selfadministered ECIG puffs, and the latency (in seconds) to initiate each puff.

Probability-Based Purchase Task (PBPT). At each session, participants completed a probability-based version of the purchase task (Roma et al. 2015; Stein \& Bickel, 2018), in which they reported the likelihood (on a scale from 1 - 100) that they would purchase an ECIG in the assigned flavor across a range of prices $(\$ 0, \$ 0.13, \$ 0.25, \$ 0.50, \$ 1, \$ 2, \$ 4, \$ 8, \$ 15, \$ 30$, 
$\$ 60, \$ 120, \$ 240$ per ECIG). Outcome measures derived from task completion include breakpoint (first price at which probability is zero), demand intensity (probability of purchase at zero), demand elasticity, and area under the curve (AUC). Demand elasticity was calculated using a modified exponential demand equation (Kofarnus, Franck, Stein \& Bickel, 2015; Stein \& Bickel, 2018) and represents the sensitivity of demand to changes in price (the proportion of change in quantity demanded to change in price). Values of elasticity $<1$ represent inelasticity of demand, where demand for a product changes little despite larger changes in price. Values $>1$ represent elasticity of demand, where demand for a product is strongly influenced by changes in price such that demand may change greatly in response to small changes in price. Finally, a demand value equal to 1 indicates that changes in demand are perfectly proportional to changes in price.

Demand for substances with high abuse potential are often inelastic, as demand for the substance remains high in spite of fluctuations in pricing (Greenwald, 2018). Finally, AUC was calculated by plotting the area under each participant's demand curve and reflects overall consumption across prices (Amlung, Yurasek, McCarty, MacKillop \& Murphy, 2015; Stein \& Bickel, 2015). Higher AUC values represent greater overall probability of purchase.

\section{Secondary Outcome Measures}

Direct Effects of Nicotine Scale (DENS). The DENS (Appendix G; Evans et al, 2006) contains 10 VAS items addressing severity of nicotine-associated side effects: "nauseous," “dizzy," "lightheaded," "nervous," "sweaty," "headache," “excessive salivation," "heart pounding," "confused," and "weak." Scale ratings range from zero to 100, with each item scored separately.

Puff topography. Puff topography was measured using video scoring. Outcomes consist of puff number, duration (measured in seconds), and IPI (measured in seconds). 
Physiological measures. Heart rate and blood pressure were measured continuously throughout each session (Noninvasive Patient Monitor model 506 NP3, Criticare Systems, Inc., Waukesha, WI). Heart rate data and blood pressure were collected every 20 seconds and every 5 minutes, respectively. Both serve as measures of exposure to nicotine (Jolma et al., 2002; Omvik, 1996) and also to verify participant safety. These materials for collecting physiological data have been used in similar work (Blank et al., 2009; Breland et al., 2002; Evans et al., 2006; Spindle et al., 2015a).

Generalized Labeled Magnitude Scale (gLMS). Participants were asked to rate the perceived intensity of three sensations (flavor sensation, harshness/irritancy, and throat hit) on a 100-point scale with seven labeled anchors (no sensation, barely detectable, weak, moderate, strong, very strong, strongest imaginable; Appendix I).

ECIG Risk Perception. Following the second and final study session, participants answered a set of three questions on their beliefs about the risks of ECIG products (Appendix E). Each item uses a rating scale that ranges from strongly disagree to strongly agree (e.g. "Electronic cigarettes are less harmful than regular cigarettes"). These questions have been adapted from previous qualitative research on ECIG attitudes, beliefs, and opinions (Choi \& Forster, 2014; Kotecha, Jawad \& Lliffe, 2016; McQueen, Tower \& Sumner, 2011). Responses are scored from zero to three and then summed and reverse scored, resulting in a possible score range of zero to nine, with higher scores representing higher risk perception.

ECIG Outcome Expectancy. Also administered at the end of the final session was the short form of the e-cigarette outcome expectancies measure (Appendix D; Pokhrel et al., 2018). This measure consists of eight items on which participants rate their perceived likelihood of experiencing certain outcomes if they were to use an ECIG (e.g.: "become more popular" or 
"hurt your lungs"). Each item is presented on a nine-point Likert scale, with responses ranging from "unlikely" to "likely." Each item corresponds to one of two factors: positive or negative outcome expectancies. Positive outcome expectancy variables $(n=4)$ are positively and significantly associated with ECIG use susceptibility (as measured by intention and willingness to use ECIGS), while negative outcome expectancy variables $(n=4)$ are inversely and significantly correlated with current ECIG use (Pokhrel et al., 2018). Items within each sub-scale (positive and negative) are summed, with possible results for each ranging from zero to 36.

Flavor Discrimination. Following administration of the ECIG Risk Perception and Outcome Expectancy measures at the final session, participants were asked to identify at which session they believed they received a fruit flavored ECIG, and at which session they believed they received the tobacco flavored electronic cigarette (Appendix J).

Exit Interview. At the end of the final session, participants were administered a semistructured interview consisting of nine questions regarding ECIG use history (e.g., typical environment during use, reasons for use) as well as four questions regarding their experience in the current research study (e.g., comparing ECIG use in the lab to naturalistic use; Appendix F).

\section{Participant Safety and Rights}

Participants' safety and rights were assured through an IRB-approved protocol enacted by trained laboratory staff. Participants were made aware of the Office of Research Integrity and Compliance, and their right to contact this office with any questions about their role as participants. No adverse events were expected for brief use of the ECIG device; however, individuals were informed of the potential risks (e.g., liquid ingredients that may serve as an allergen). Heart rate and blood pressure were monitored continuously throughout each session to ensure participant safety. A medical monitor was available for contact if a participant's heart rate 
fell out of the range of 50 to $110 \mathrm{bpm}$, systolic blood pressure out of the range of 90 to 150 $\mathrm{mmHg}$, or diastolic blood pressure out of the range of 60 to $100 \mathrm{mmHg}$. Confidentiality was ensured through the use of coded identity numbers for participant data. Data was stored in locked rooms and on password protected computers.

\section{Data Analysis}

Values for heart rate and systolic and diastolic blood pressure were computed by averaging across the 5 minutes preceding and the 5 minutes following a directed bout in order to obtain pre- and post-bout values. Subjective questionnaire (i.e., DEPS and DENS) and physiological data were analyzed using 3-factor, repeated measures analyses of covariance (ANCOVAs): condition (two levels: tobacco and fruit flavor) $\mathrm{x}$ bout (two levels: bouts 1 and 2) $\mathrm{x}$ time (two levels: pre- and post-bout). Covariates included ECIG lifetime use (both subjective and physiological outcomes), as well as risk perceptions and expectancies (subjective outcomes only). These covariates were selected due to their potential for influencing select outcome variables. ECIG lifetime use may affect both subjective and physiological outcomes by influencing the length of ECIG puffs, as more experienced users tend to take longer puffs resulting in higher nicotine delivery (Farsalinos et al., 2015; Hiler et al., 2017). Further, among youth and young adults, greater ECIG use experience is associated with higher positive expectancies as well as lower negative expectancies and risk perception (Bernat et al., 2018; Chafee et al., 2015). In turn, ECIG risk perception and expectancies may influence participants' willingness to engage with the ECIG device. For example, individuals with higher risk perception and negative expectancies may be less willing to engage with the ECIG product (Leung et al., 2018) thus reducing the likelihood of engagement during behavioral economics tasks. 
Puff topography and gLMS data were also analyzed using repeated measures ANCOVAs, but without the time factor included (i.e., condition $\mathrm{x}$ bout). Note that gLMS data are based on $\mathrm{n}=17$, as this measure was added to the protocol later in the data collection phase. These same analyses sans covariates were conducted using session order (1 and 2) instead of flavor (fruit and tobacco) for the condition factor to examine potential order effects. Finally, mixed repeatedmeasures ANOVAs for all measures, including behavioral economic task outcomes, were conducted to examine differences due to fruit choice, flavor order, gender, and race, respectively. Given limited variability of race $(n=25$ White, $n=5$ non-White), the variable was dichotomized. Of 175 possible main and interaction effects involving each variable as a betweensubjects factor, 5 were significant for fruit choice, 9 for flavor order, 9 for gender, and 15 for race. Given the paucity of significant effects for fruit choice, flavor order, and gender $(\leq 5 \%)$ and lack of consistent and meaningful patterns of significance, results for these analyses are not described further. For significant analyses, differences between means were examined using Tukey's Honestly Significant Difference (HSD; $p<.05)$. Descriptive statistics are provided for flavor discrimination, fruit choice, lifetime product use, ECIG risk perception, and EIG expectancy data. As with the gLMS, flavor-discrimination questions were only completed by 17 participants given the delay in inclusion of the measure. All other measures include data from all 30 participants. Finally, responses to the exit questionnaire were summarized.

\section{Study Hypotheses}

Some study results were predicted based on previous research on similar topics. The hypotheses were as follows: For subjective items, fruit flavored liquid were hypothesized to be rated more pleasurable than tobacco flavored liquid as evidenced by higher ratings of items such as "taste good," "satisfying," and "liking" (as in Audrain-McGovern, Strasser \& Wileyto, 2016; 
Goldenson et al., 2016; Kim et al, 2016). For the behavioral economic tasks, participants were hypothesized to work harder (i.e., have higher break points on the progressive ratio task) for fruit flavored liquid as opposed to tobacco flavored liquid (as in Audrain-McGovern, Strasser \& Wileyto, 2016), and to be significantly more likely to purchase fruit flavored liquid at higher prices than tobacco flavored liquid (i.e., will have higher crossover points on the probabilistic purchase task; as in Barnes et al., 2017; Goldenson et al., 2016). Secondary outcome measures were exploratory, and thus no formal hypotheses were proposed.

\section{Results}

\section{Demographics}

Of 41 total participants recruited, eight were excluded $(n=4$ for inconsistent reporting at screening; $\mathrm{n}=2$ for expired air $\mathrm{CO}>5 \mathrm{ppm} ; \mathrm{n}=2$ for urinary cotinine values $>100 \mathrm{ng} / \mathrm{mL}$ ). Three participants dropped out of the study following enrollment, leaving a final sample of 30 study completers. Participant baseline characteristics for these 30 participants are displayed in Table 1. Those young adults enrolled were largely college students, Caucasian, and female, with a range of 3 to 50 lifetime ECIG uses (median $=10$; mode $=10$ ). Their choice between fruit medley and mango flavors were relatively comparable.

\section{Primary Outcome Measures}

For all primary and secondary subjective, physiological, and topography outcomes, results for repeated measures ANCOVAs are displayed in Table 2. Due to the large number of main and interaction effects adjusted for covariates, and the very few significant findings, results for factors by each covariate are not provided in table format. Rather, these results are described in the below text as relevant. Results for mixed repeated-measures ANOVAs that include race as a between-subjects factor are displayed in Table 3. Repeated measures ANOVAs that describe 
order effects are displayed in Table 4. Finally, mixed repeated measures ANOVAs for behavioral economics outcomes are included in Table 5.

Direct Effects of Products Scale. As shown in Table 2, few main or interaction effects were observed for the DEPS items. A significant main effect of bout (i.e., collapsed across flavor and time factors) was detected for "pleasant," such that participants rated the ECIG as significantly more pleasant at the second $(M=25.0, S E=3.2)$ compared to the first $(M=13.5$, $S E=1.7)$ puffing bout of each session, $F(1,25)=4.31, p=.048$, partial $\eta 2=0.15$. For the items "taste good" and "calm," a significant bout x time interaction was observed ( $F$ 's > 5.45, $p$ 's < 0.05 , partial $\eta 2>0.18)$. These effects also were significant for the bout $\mathrm{x}$ time $\mathrm{x}$ risk interaction, $F$ 's $>4.53, p$ 's $<0.05$, partial $\eta 2>0.15$. Mean “taste good" scores were $0.1(S E=0.06)$ and 33.5 $(S E=4.7)$ at pre- and post-bout 1 , respectively, and $23.3(S E=4.6)$ and $32.9(S E=4.9)$ at preand post-bout 2 , respectively. Scores were significantly different between pre- and post-bout for bout 1 but not bout 2, as were scores between pre-bouts 1 and 2 (Tukey's HSD, $p<0.05$ ). A similar pattern was observed for "calm," where mean scores were $0.12(S E=0.05)$ and 24.98 (SE $=3.3)$ at pre- and post-bout 1 , respectively, and $18.2(S E=3.9)$ and $23.8(S E=3.8)$ at pre- and post- bout 2, respectively (Tukey’s HSD, $p<0.05$ ).

When examining effects as a function of race (Table 3), a bout $\mathrm{x}$ race interaction was revealed for "sick" ratings, $F(1,28)=7.47, p=.011$, partial $\eta 2=0.21$, where significant increases in sickness were reported between bout 1 and bout 2 for non-White $(M=14.0, \mathrm{~S} E=$ 4.5, and $M=35.6, S E=8.9$, respectively; Tukey's HSD, $p<.05)$, but not White participants $(M$ $=7.6, \mathrm{~S} E=1.99$, and $M=11.1, S E=3.96$, respectively; Tukey's HSD, $p>.05)$. Further, "sick" ratings at bout 2 were significantly higher for non-White participants than White participants (Tukey's HSD, $p<.05)$. For “dizzy," there was a bout $\mathrm{x}$ time $\mathrm{x}$ race interaction, $F(1,28)=4.84$, 
$p=.036$, partial $\eta 2=0.15$ (see Figure 2). For both White and non-White individuals, ratings increased significantly in bout 1 (Tukey's HSD, $p<0.05$ ) but not bout 2 (Tukey's HSD $p>.05$ ). Further, post-bout 2 ratings for non-White participants were significantly higher than either postbout rating for White participants (Tukey's HSD, $p<.05$ ). Finally, a flavor $\mathrm{x}$ bout $\mathrm{x}$ time $\mathrm{x}$ race interaction was present for "pleasant," $F(1,28)=6.12, p=.020$, partial $\eta 2=0.18$ (see Figure 3). For White individuals, ratings of "pleasant" increased significantly from pre- to post-bouts 1 and 2 within each flavor (Tukey's HSD, $p<0.05$ ). For non-White individuals, the fruit flavored ECIG produced increases from pre-to post- bout that were significant for bout 1 (Tukey's HSD, $p<.05$ ), but not bout 2 (Tukey's HSD, $p>.05$ ). For the tobacco flavored ECIG, ratings were significantly increased pre- to post- bout 1 (Tukey's HSD, $p<.05$ ), but significantly decreased pre-to post-bout 2 (Tukey's HSD, $p<.05$ ). Further, ratings of "pleasant" were significantly higher for White participants post-bouts 1 and 2 with the fruit flavored ECIG, and post-bout 2 with the tobacco flavored ECIG, compared to non-White participants (Tukey's HSD, all $p$ 's < .05). Finally, pre-bout 2 with the tobacco flavored ECIG, non-White participants had significantly higher ratings of "pleasant" than White participants (Tukey’s HSD, $p<.05)$.

For the effects of session order (see Table 4), there was a significant main effect of order for "reduced hunger," $F(1,29)=5.40, p=0.027$, partial $\eta 2=0.16$, such that hunger reduction was rated higher at the first session $(M=20.17, S E=3.59)$ than at the second session $(M=$ 14.53, $S E=3.58 ; p<0.05)$. There was also significant main effect of bout for "concentrate," $F$ $(1,29)=9.38, p=0.01$, partial $\eta 2=0.24$, such that values were significantly higher at bout $2(M$ $=13.4, S E=3.4)$ than at bout $1(M=5.5, S E=1.3)$. There was also a main effect of time for “concentrate", $F(1,29)=15.32, p=0.001$, partial $\eta 2=0.35$, where "concentrate" was rated higher post-bout $(M=13.8, S E=3.1)$ than at pre-bout $(M=5.2, S E=1.6)$. An order $\mathrm{x}$ time 
interaction was present for both ratings of "calm" and "sick," $\mathrm{F}$ 's $>7.41, p$ 's $<.05$, partial $\eta 2>$ 0.20. For "calm", mean ratings were $10.4(S E=2.3)$ and $29.2(S E=4.8)$ pre- and post- bouts for session 1 , respectively, and $7.9(S E=2.1)$ and $19.6(S E=4.1)$ pre- and post- bouts for session 2 , respectively. Post-bout ratings were significantly higher than pre-bout ratings for both sessions, and post-bout ratings for session 1 were significantly higher than those for session 2 (Tukey's HSD, $p<.05)$. The same pattern emerged for ratings of "sick", for which mean ratings were 6.3 $(S E=2.1)$ and $22.1(S E=4.9)$ pre- and post- bouts for session 1 , and $5.6(S E=1.9)$ and $13.7(S E$ = 3.8; Tukey's HSD, $p<.05$ ). Finally, significant bout $\mathrm{x}$ time interaction effects were present for all DEPS ratings other than "concentrate" (F's $>8.56$, p's $<0.05$, partial $\eta 2>0.23$ ) such that scores increased significantly from pre- to post-bout 1 but not bout 2 (Tukey's HSD, $p<.05$ ). For "taste good," the measure with the largest $F$ value for this interaction $(F(1,29)=23.96, p=$ .000 , partial $\eta 2=0.45)$ means were $0.12(S E=0.06)$ and $33.5(S E=4.6)$ pre- and post- bout 1 , respectively, and $23.3(S E=4.7)$ and $32.97(S E=4.97)$, respectively.

Progressive Ratio Task. Table 5 displays mixed repeated-measures ANOVA results for PRT analyses with race included as a between-subjects factor. No significant difference was revealed between flavor conditions on any PRT outcomes, including when considered as a function of race (i.e., rewards earned, breakpoint, total responses, responses per minute, and latency to puff; $F \mathrm{~s}<3.84, p$ 's $>0.05$, partial $\eta 2<0.12$ ). Descriptive statistics for all PRT outcomes for the main effect of flavor condition are displayed in Table 6.

Probability Based Purchase Task. Table 5 also depicts mixed repeated-measures ANOVA results for all PBPT outcomes (i.e., breakpoint, demand intensity, demand elasticity, and AUC). Descriptive statistics for all PBPT outcomes are displayed in Table 6. No significant differences were found between flavor conditions for any outcome measure, or between flavor 
condition $\mathrm{x}$ race $(F s<0.27, p$ 's $>0.05$, partial $\eta 2<0.01)$. Graphs of participant demand curves for fruit and tobacco flavored ECIGs are included in Figures 4 and 5, respectively.

\section{Secondary Outcome Measures}

Direct Effects of Nicotine Scale. Table 2 displays ANCOVA results for all DENS items. No significant main effects of flavor, bout, or time were revealed ( $F^{\prime}$ 's $<2.42, p$ 's $>0.05$, partial $\eta 2<0.09)$. A significant flavor $x$ time interaction effect was revealed for "dizzy," $F(1,25)=$ 4.85, $p=0.037$, partial $\eta 2=0.16$. Post-hoc comparisons (Tukey's HSD, $p<.05$ ) revealed that ratings of dizziness were significantly higher post-bout compared to pre-bout for both fruit $\left(M_{\text {pre- }}\right.$ bout $\left.=3.97, S E=1.3 ; M_{\text {post-bout }}=18.9, S E=4.1\right)$ and tobacco flavors $\left(M_{\text {pre-bout }}=4.98, S E=1.8\right.$; $\left.M_{\text {post-bout }}=17.2, S E=4.1\right)$. No differences were revealed between flavor conditions $($ Tukey's HSD, $p$ 's > .05). Finally, there was a significant interaction effect of flavor $\mathrm{x}$ bout $\mathrm{x}$ time for “confused," $F(1,25)=4.75, p=0.039$, partial $\eta 2=0.16$, as well as for the interaction between these factors and the risk covariate $F(1,25)=9.56, p=.005$, partial $\eta 2=0.28$ (see Figure 6 ). Ratings of confused significantly increased from pre- to post-bout 1 within each flavor condition (Tukey's HSD, $p<.05$ ); however, these increases were more pronounced for the first bout compared to the second bout. For bout 2, Tukey’s post-hoc tested revealed significant differences for pre-post bout for tobacco flavor (Tukey’s HSD, $p<0.05$ ), but no other meaningful differences.

When examining race as a between-subjects factor (Table 3), there was a significant interaction effect of flavor x race for "sweaty," $F(1,28)=6.68, p=.015$, partial $\eta 2=0.19$. NonWhite individuals provided significantly lower ratings of sweatiness when using the tobacco ( $M$ $=8.3, S E=5.9)$ compared to the fruit flavored ECIG $(M=18.0, S E=7.6$; Tukey's HSD, $p<$ $.05)$, while White individuals reported comparable ratings between flavors $(M=5.5, S E=3.4$ 
and $M=5.5, S E=2.6$, respectively; Tukey's HSD, $p>.05)$. Non-White participants also had significantly higher ratings of "sweaty" than White participants when using the fruit flavored ECIG (Tukey's HSD, $p<.05)$. For “dizzy," there was a significant time x race interaction, $F(1$, 28) $=6.25, p=.019$, partial $\eta 2=0.18$, where there were significantly higher post-bout ratings of dizziness $(M=36.3, S E=8.6)$ compared to pre-bout $(M=7.1 S E=2.99)$ for non-White individuals (Tukey's HSD, $p<.05)$, but not for White individuals $(M=14.4, S E=3.8$ and $M=$ 3.96, $S E=1.3$, respectively; Tukey's HSD, $p>.05)$. Post-bout, non-White participants had significantly higher ratings of "dizzy” than White participants (Tukey’s HSD, $p<.05$ ). A significant flavor $\mathrm{x}$ bout $\mathrm{x}$ race interaction was present for "nauseous," $F(1,28)=5.67, p=.024$, partial $\eta 2=0.17$ (see Figure 7). For non-White individuals, mean ratings increased significantly from bout 1 to bout 2 for the tobacco-flavored ECIG only (Tukey's HSD, $p<.05$ ). Further, bout 2 ratings with the tobacco flavored ECIG were significantly higher for non-White participants than White participants (Tukey's HSD, $p<.05$ ). For "lightheaded," there was a significant effect of flavor $\mathrm{x}$ time $\mathrm{x}$ race, $F(1,28)=5.25, p=.03$, partial $\eta 2=0.16$, where ratings of lightheadedness significantly increased from pre- to post- bouts for all participants across races and flavors (see Figure 8; Tukey's HSD, $p<.05$ ). Post-bout with the fruit flavored ECIG, nonWhite participants had significantly higher ratings of lightheadedness compared to White participants (Tukey's HSD, $p<.05$ ). Finally, there was a significant bout $\mathrm{x}$ time $\mathrm{x}$ race interaction for "headache," $F(1,28)=4.92, p=.035$, partial $\eta 2=0.15$ (see Figure 9). For White individuals, there were no significant differences in means pre- to post-bout 1 or bout 2 (Tukey's HSD, $p>.05)$. However, for non-White individuals, there were significant increases in means pre- to post-bout 1 and bout 2 (Tukey's HSD, $p<.05$ ). Post-bout means were significantly higher for non-White participants than for White participants (Tukey’s HSD, $p<.05$ ). 
Significant main effects of order (i.e., collapsed across bout and time factors) were present for ratings of "dizzy," "lightheaded," "nauseous," and "weak" ( $F$ 's $>4.70, p$ 's $<0.05$, partial $\eta 2>0.14$ ) (see Table 4). For all four DENS items, ratings were significantly higher at session 1 than at session 2. For "lightheaded," the item with the largest $F$ value for the main effect of order $(F(1,29)=12.44, p=.001$, partial $\eta 2=.30)$, mean scores at session 1 were 19.0 $(S E=2.8)$ compared to $11.3(S E=2.1)$ at session 2. Significant main effects of bout were present for ratings of "lightheaded," "nervous," and "heart pounding" ( $F$ 's $>4.35, p$ 's $<0.05$, partial $\eta 2>0.13$ ). Participants reported significantly higher levels of "lightheadedness" at bout 2 $(M=17.2, S E=2.6)$ compared to bout $1(M=13.1, S E=2.2)$. The opposite trend was revealed for "nervous" and "heart pounding" where ratings at bout $1(M=9.3, S E=2.7$ and $M=8.9, S E=$ 2.4, respectively) were higher than at bout $2(M=6.6, S E=2.2$ and $M=6.9, S E=1.8$, respectively). Main effects of time were also present for "dizzy," "lightheaded," "nauseous," and “weak" ( $F$ 's $>4.51, p$ 's $<0.05$, partial $\eta 2>0.14)$. For all four outcomes, scores were higher post- bout compared to pre- bout. For "lightheaded," the item with the largest $F$ value for the main effect of time $(F(1,29)=33.09, p=.00$, partial $\eta 2=.53)$, mean pre- bout scores were 5.8 $(S E=1.2)$ compared to $24.5(S E=3.7)$ post-bout. Finally, there was a bout $\mathrm{x}$ time interaction for "sweaty" $(F(1,29)=5.92, p=0.02$, partial $\eta 2=0.17)$, though post-hoc tests did not reveal significant differences between time points at either bout (Tukey's HSD $p>.05$ ). For bout 1 , mean pre- to post-bout sweaty ratings were $7.8(S E=3.2)$ and $6.98(S E=2.7)$, respectively. For bout 2 , these values were $5.0(S E=2.7)$ and $7.3(S E=2.7)$, respectively.

Puff Duration. No significant main or interaction effects were revealed for puff duration ( $F$ 's $<0.85, p$ 's $>0.05$, partial $\eta 2<0.03$; Table 2$)$. Collapsed across time, mean puff duration was 2.4 seconds $(S E=0.2)$ for fruit flavor and 2.5 seconds $(S E=0.2)$ for tobacco flavor. No 
significant effects were revealed when including race as a between-subjects factor $\left(F^{\prime} \mathrm{s}<0.84\right.$, p's $>0.05$, partial $\eta 2<0.03$; Table 3).

For the effects of session order (see Table 4), there was a significant main effect of bout $[F(1,29)=11.27, p=0.002$, partial $\eta 2=0.28]$, such that participants took longer puffs at bout 2 $(M=2.5$ seconds, $S E=0.2)$ than at bout $1(M=2.3, S E=0.2)$.

Physiological Measures. A significant main effect of flavor (collapsed across bout and time factors) was revealed for systolic blood pressure $(F(1,26)=5.34, p=0.029$, partial $\eta 2=$ 0.17; Table 2), where systolic pressure was higher for the tobacco condition $(M=120.4 \mathrm{mmHg}$, $S E=2.2)$ than for the fruit condition $(M=116.8 \mathrm{mmHg}, S E=2.6)$. Systolic blood pressure also revealed a significant bout $\mathrm{x}$ time interaction $(F(1,26)=6.46, p=0.017$, partial $\eta 2=0.20)$ including when accounting for the covariate of lifetime ECIG use, $\mathrm{F}(1,26)=12.64, p=0.002$, partial $\eta 2=0.32$. However, post-hoc tests did not reveal any significant differences in means across time points or bouts (Tukey’s HSD, $p>$.05). Mean systolic blood pressure was 116.1 $\mathrm{mmHg}(S E=2.8)$ pre-bout 1 compared to $120.2 \mathrm{mmHg}(S E=2.4)$ post-bout 1 , and 116.98 $\mathrm{mmHg}(S E=2.6)$ pre-bout 2 compared to $120.5 \mathrm{mmHg}(\mathrm{SE}=2.1)$ post-bout 2 . There was a significant main effect of time for heart rate, $F(1,25)=7.44, p=0.011$, partial $\eta 2=0.22$, where heart rate was significantly higher post-bout $(M=85.5 \mathrm{bpm}, S E=1.8)$ compared to pre-bout $(M$ $=82.3 \mathrm{bpm}, S E=1.5)$.

When examining race as a between-subjects factor (see Table 3), a significant interaction effect of flavor $\mathrm{x}$ bout $\mathrm{x}$ race was revealed for diastolic blood pressure, $F(1,26)=5.68, p=.025$, partial $\eta 2=.18$ (see Figure 10). For differences between conditions and bouts, bout 2 was significantly higher than bout 1 for non-White participants using the fruit flavored ECIG only (Tukey’s HSD, $p<.05$ ). Non-White participants had significantly higher diastolic pressure than 
White participants at bout 2 using the fruit flavored ECIG and at bout 1 using the tobacco flavored ECIG (Tukey's HSD, $p<.05$ ). An interaction effect of bout $\mathrm{x}$ time $\mathrm{x}$ race was revealed for heart rate, $F(1,26)=11.05, \mathrm{p}=.003$, partial $\eta 2=.30$ (see Figure 11). Across racial groups, there was a significant heart rate increase pre- to post- bout 1 (Tukey's HSD, $p<.05$ ) but not bout 2 (Tukey's HSD, $p>.05$ ). Additionally, pre-bout 1 White participants had significantly higher heart rates than non-White participants (Tukey's HSD, $p<.05$ ). Further, interaction effects of flavor $\mathrm{x}$ bout $\mathrm{x}$ time $\mathrm{x}$ race were revealed for all three physiological outcome measures $(F \mathrm{~s}>4.65, p \mathrm{~s}<.05$, partial $\eta 2>0.15)$. For heart rate, pre-post bout values increased significantly for all but non-White individuals at bout 2 using the tobacco flavored ECIG (see Figure 12; Tukey's HSD, $p<.05$ ). For systolic blood pressure, only differences in pre-post bout 1 values for non-White individuals using the tobacco flavored ECIG were significant (see Figure 13; Tukey’s HSD, $p<.05)$. Further, non-White individuals had significantly higher systolic blood pressure readings for the majority of time points measured (i.e. for fruit: pre-bout 1, prebout 2, post-bout 2, and for tobacco: post-bout 1, pre-bout 2; Tukey's HSD, $p<.05$ ). Finally, for diastolic blood pressure, non-White participants had significantly higher diastolic blood pressure readings than White participants post-bout using the fruit flavored ECIG, as well as post-bout 1 and pre-bout 2 with the tobacco flavored ECIG (See Figure 14; Tukey's HSD, $p<.05$ ).

When analyzing order effects (see Table 4), no significant main effects of order were detected for any physiological outcome measure $(F \mathrm{~s}<1.84$, $p$ 's $>0.05$, partial $\eta 2<0.06)$. Significant main effects of time were present for both systolic and diastolic blood pressure $(F$ 's > $6.41, p$ 's $<0.05$, partial $\eta 2>0.19)$, such that values were significantly higher post-bout $(M=$ $120.4 \mathrm{mmHg}, S E=2.1$ and $M=71.2 \mathrm{mmHg}, S E=1.3$, respectively $)$ compared to pre-bout $(M=$ $116.5 \mathrm{mmHg}, S E=2.5$ and $M=68.9 \mathrm{mmHg}, S E=1.3$, respectively). An interaction effect of 
order $\mathrm{x}$ bout $\mathrm{x}$ time was detected for heart rate, $F(1,27)=12.61, p=.001$, partial $\eta 2=0.32$ (see Figure 15). Generally, heart rate increased from pre- to post-bouts 1 and 2 within each session. Heart rate was significantly higher post-bout than pre-bout across sessions and bouts (Tukey's HSD, $p<0.05$ ), with the exclusion of post-bout 2 in session 1 (Tukey's HSD, $p>0.05$ ). Additionally, heart rate post-bout 2 at the first study session was significantly lower than heart rate post-bout for all other puffing bouts across sessions (Tukey's HSD, $p<0.05$ ).

Generalized Labeled Magnitude Scale. Table 2 shows no significant main or interaction effects were revealed for any gLMS item $(F$ 's $<4.09, p$ 's $>0.05$, partial $\eta 2<0.26)$. Collapsed across time, the mean rating of flavor sensation was $30.3(S E=4.5)$ for fruit flavor and $26.7(S E=5.1)$ for tobacco flavor. For harshness, mean ratings were $42.7(S E=5.8)$ for fruit and $34.7(S E=5.2)$ for tobacco, and mean ratings of throat hit were $43.5(S E=5.96)$ for fruit and $38.1(S E=6.0)$ for tobacco. A significant bout $\mathrm{x}$ race interaction (Table 3) was detected when examining race as a between-subjects factor for sensation, $F(1,15)=4.73, p<.05$, partial $\eta 2=$ 0.24. For White participants, ratings were $28.8(S E=4.7)$ and $32.8(S E=4.7)$ for bouts 1 and 2 , respectively, and $20.0(S E=10.1)$ and $15.0(S E=10.2)$ for non-White participants, respectively. For both bouts, White participants had significantly higher ratings of sensation than non-White participants (Tukey's HSD, $p<0.05$; Table 3). There were no significant effects for the gLMS measure involving the session order factor $\left(F^{\prime} \mathrm{s}<0.07, p\right.$ 's $>0.05$, partial $\eta 2<0.01$; see Table 4).

ECIG Risk Perception. The mean perceived risk score for participants was $5.3(S D=$ 1.4), on a scale that ranged from 0 to 9 . Average scores were relatively low for two of the three items, reflecting agreement with statements suggesting that ECIGs can be used as a smoking cessation tool $(M=1.6, S D=0.8)$ and as a harm reduction product $(M=1.2, S D=0.8)$. 
However, participants generally did not agree with the statement that ECIGs are less addictive than cigarettes $(M=2.5, S D=0.7)$

ECIG Outcome Expectancies. Overall, participants reported higher negative ECIG outcome expectancies $(M=21.7, S D=4.4)$ than positive $(M=11.8, S D=7.0)$. Means and standard deviations for ECIG outcome expectancies, including individual items, are depicted in Table 9.

Flavor Discrimination. The majority of participants $(82.35 \% ; n=14$ of 17$)$ who were asked to identify the order of ECIG flavors used by visit responded correctly.

Exit Interview. Table 8 displays flavor preferences and frequencies for all participants. Overall, participants described liking flavors that were sweet/fruity and did not burn. The top preferred flavors included mint/menthol $(n=10)$, mango $(n=7)$, and berry/berry medley $(n=5)$. Some participants also reported favoring flavors modeled after sugary drinks (i.e., pink lemonade, grape soda; $\mathrm{n}=2$ ) and cereals (i.e., fruit loops and fruity pebbles; $\mathrm{n}=2$ ). Six participants reported a dislike of all flavors that they have tried (i.e., prefer no flavors). Among the flavors most disliked by participants were mint/menthol $(n=9)$ and tobacco $(n=8)$, though the majority stated that they do not dislike any flavors $(n=10)$.

Participant responses to the exit interview, which are categorized according to themes, are presented in Table 9. Responses from the participants who provided a reason for liking or disliking flavors, as described above, are summarized. All 30 participants reported that their ECIG use occurred primarily or exclusively in social settings. The most common reasons for using an ECIG included were curiosity $(n=17)$, social pressure $(n=16)$, and flavor $(n=11)$. Note that some participants listed more than one reason for use. Interestingly, only 4 participants described using an ECIG because they enjoyed the experience (e.g. sensory or physiological). 
Participants also responded to several questions regarding their study experience. Overall, the majority of participants $(n=22)$ reported that the amount of product used in the study session was more than they would normally consume in their natural environment. Self-reported reasons for participating in the study included compensation $(n=19)$ and a desire to contribute to research $(n=9)$. Some participants $(n=6)$ noted that they felt that it was important to further research on electronic cigarettes, in particular. Aspects of the study that participants most frequently reported disliking included the long wait in between tasks $(n=5)$, having to use the ECIG $(n=3)$, and the length of study sessions $(n=2)$. Participants reported that the study room was comfortable $(n=6)$. Some participants noted that they enjoyed having time to rest in between sessions $(n=2)$ and using the ECIG as part of the study $(n=2)$. Note that $43 \%$ and $57 \%$ of participants did not report any aspects of the study which they disliked or liked, respectively.

\section{Discussion}

The risk of ECIG product initiation by tobacco-naïve youth and young adults remains a pressing public health concern in the United States. In light of the recent documented increase in ECIG use among youth and young adults (Gentzke et al., 2019; Dai \& Leventhal, 2019), the FDA has identified the use of non-tobacco flavors that are appealing to youth as a target for potential product regulation (FDA Regulation of Electronic Nicotine Delivery Systems and Investigation of Vaping Illnesses, 2019). Cross-sectional research largely supports the theory that flavored ECIG products are particularly appealing to this population, as the majority of them report initiating ECIG use with a sweet flavored product (McKelvey, Biaocchi \& HalpernFelsher, 2018). The use of a sweet (vs tobacco) flavored ECIG, particularly during the period of initial use, may increase later tobacco use due to a) reduction of the initial aversive effects of nicotine, b) learned association between reinforcing a olfactory cue and nicotine delivery, and c) 
metabolic properties of certain flavors that may increase the bioavailability of nicotine (Wickham, 2015). These findings have been capitalized on by the tobacco industry in the past to recruit new generations of users of other tobacco products (e.g., smokeless, cigars) (David Weiss Associates, 1987; Kostygina \& Ling, 2016). Despite this, limited experimental work exists on the role of flavoring in ECIG abuse liability among tobacco-naïve young adults. As such, the findings of the current study can help to complement existing research in order to inform future regulatory decisions.

\section{Nicotine Delivery}

A key factor in the conceptualization of flavor as a driver of abuse liability of tobaccoproducts lies in its ability to mask the harsh sensory experience (e.g., throat burning, taste) associated with tobacco/nicotine effects (Carpenter et al., 2005; Cummings et al., 2002). Thus, examining the abuse liability of flavored ECIGs requires the use of a device capable of nicotine delivery. In the current study, measures of heart rate, which are commonly used markers of nicotine delivery (Jolma et al., 2002; Omvik, 1996), were increased significantly from pre- to post-ECIG bouts within each flavor condition. Increases in heart rate in response to a standardized 10-puff ECIG bout have been reported in previous work using JUUL with ECIGnaïve cigarette smokers, where heart rate increased from $63.7 \mathrm{bpm}(S E=2.7)$ to $73.9 \mathrm{bmp}(S E=$ 2.4) from pre- to post-bout respectively (Maloney et al., 2019b). This effect is also seen for ECIG experienced users following a standardized 10-puff bout with an older generation of ECIG (Spindle et al., 2018). Differences in heart rate were less pronounced in the current study (differences of about $3 \mathrm{bpm}$ ) compared to differences found in prior work (7 - $13 \mathrm{bpm}$; Maloney et al., 2019a; Maloney et al., 2019b; Spindle et al., 2018). Taken together, these data suggest that the JUUL device is capable of delivering physiologically active doses of nicotine to tobacco- 
naïve individuals, albeit at lower levels compared to those demonstrated in this previous work with ECIG experienced users and ECIG-naïve cigarette smokers. Differences across studies may be due to differences in the device characteristics that were used (e.g., liquid nicotine concentration; battery power) and/or the puff topography patterns observed (e.g., size and/or volume of puffs).

\section{Subjective Ratings of ECIGS}

Overall, few significant effects emerged for subjective ratings as a function of ECIG flavor. One possible reason for this pattern is a floor effect, as ratings across subjective measures were notably low for both flavors. For example, mean ratings for 100-point VAS subjective items (e.g. lightheaded, nauseous, taste good, pleasant) ranged from 3.1 to 22.5 (SD's 9.1 to 27.9). These findings contribute to the currently mixed literature, which is largely based on adult populations with varied ECIG and cigarette use histories. For instance, no differences were found in subjective ratings of enjoyment between strawberry and tobacco ECIG flavors in adult ECIG-experienced users with minimal cigarette smoking history (St. Helen et al., 2017). In contrast, another study found that hedonic ratings of sweet ECIG flavors (e.g., piña colada, peach schnapps) were higher than for non-sweet flavors (e.g., classic tobacco) in adult experienced ECIG users or dual users of both ECIGs and cigarettes (Kim et al., 2016). Further, a study assessing subjective appeal among young adult JUUL users (18 - 25 years) found that young adults rated their "preferred" flavored ECIGs (71.4\% mint, $14.3 \%$ menthol, $14.3 \%$ mango) as significantly more favorable (e.g., "wanting more") than tobacco flavored ECIGs (Pacek, Kozink, Wimbish \& McClernon, 2019). Notably, no experimental work has been completed within tobacco-naïve users in this age group. As such, some of these differences in findings may be attributed to differences in the study sample, given that tobacco-naïve young adults 
demonstrate low subjective ratings of ECIG products, overall. Additionally, differences between study results may be related to differences in ECIG model, flavors, nicotine concentration, and PG/VG ratios used.

\section{Behavioral Economic Outcomes}

Outcomes for tasks measuring willingness to pay and work for the ECIG (e.g. PBPT, PRT) also did not reveal significant differences between flavors. These findings differ from prior work in young adult JUUL users $(18-25$ years $)$, in which participants completed a concurrent choice task in which they could expend effort for fixed doses of JUUL vapor in either a "preferred" flavor (e.g., mint, menthol, or mango) or tobacco flavor. Effort required to earn a dose from the preferred flavored pod was on an escalating schedule while effort required for the tobacco flavored pod was on a fixed schedule. Participants made greater choices (i.e., expended greater effort by responding more) for preferred compared to tobacco flavored pods (7.9 vs. 3.6; Pacek, Kozink, Wimbish \& McClernon, 2019). Findings from the present study also differ from previous work in adult experienced ECIG users who report using < 15 tobacco cigarettes per day, which found that participants were willing to pay more for sweet compared to non-sweet flavors (Goldenson et al., 2016). Other studies have found differences in purchase task and PRT outcomes for flavored compared to non-flavored ECIGs among cigarette smokers (AudrainMcGovern, Strasser \& Wileyto, 2016; Barnes et al, 2017). Barnes et al. (2017), for example, used a cigarette purchase task in a sample of smokers with no history of regular ECIG use (never used weekly for $>1$ month). Participants were asked to provide the number of times that they would be willing to take 10-puffs from a tank-style (second generation) ECIG at a range of prices (per $\$ 10$ puffs) from $\$ 0$ to $\$ 10.24$. Mean demand intensity for participants was 0.04 for the cherry flavored ECIG and 0.11 for the unflavored ECIG. These patterns are similar to data 
from the current study where mean demand elasticity was $0.0004(S D=.002)$ for the fruit flavored ECIG and 0.001 (0.007) for the tobacco flavored ECIG. However, the latter elasticity values are considerably lower. While this suggests inelasticity of demand, which is usually considered a marker of abuse liability, these values are a function of low probability of purchase across prices rather than high probability. This is demonstrated in the present study by AUC values of $0.06(0.08)$ for both flavors, indicating that probability of consumption across participants and flavors was only $6 \%$ of total possible consumption. In contrast to the present PBPT findings, though, Barnes et al. (2017) did reveal significant differences in demand intensity, where participants were reported willingness to take 10.2510 -puff bouts of cherry flavored and 8.44 10-puff bouts of the unflavored ECIG when the price was $\$ 0$. Though assessing two flavored ECIGS, participants from the present study did not differ on probability of purchase at $\$ 0$ between fruit and tobacco flavored products $(\mathrm{M}=68.9, \mathrm{SD}=77.97$ and $\mathrm{M}=$ $75.1, \mathrm{SD}=83.98$, respectively). PRT data in the current study also differs from prior work by Audrain-McGovern et al. (2016) in which smokers (with at least one reported ECIG use) worked harder for flavored compared to unflavored ECIG puffs, during a 10-trial task in which they could respond on a fixed-ratio (FR) 25 schedule of responding (via mouse clicks) for a puff from an unflavored ECIG or on a progressive-ratio (PR) schedule of $25 \mathrm{x}$ for a puff from a flavored ECIG. Breakpoint for flavored puffs was 597 responses compared to 127 responses for unflavored, and participants took twice as many puffs from the flavored, compared to the unflavored ECIG (40 vs 23 puffs). In comparison, participants from the present study took an average of 3.5 (5.7) puffs from the fruit flavored ECIG and 3.5 (6.1) from the tobacco flavored ECIG and had breakpoints of 112.8 (243.2) and 136.2 (298.4), respectively. 
The discrepancy in findings between the present study and prior work may be the result of an important difference between samples of regular ECIG or cigarette users and one that is tobacco-naïve - the motivating presence of nicotine withdrawal. Indeed, for two of the three aforementioned studies, participants were required to abstain from product use overnight in order to induce nicotine withdrawal. In the present study, only 13 participants made enough responses to earn at least one reward (one puff from the ECIG) at both sessions. Further, low overall subjective ratings for product liking suggest a potential floor effect in which participants' willingness to work for either product is too low to distinguish meaningful differences between the two. Given this, and the lack of nicotine withdrawal, it stands to reason that many participants were not motivated to pay or work for either ECIG flavor.

\section{Race Effects}

For physiological measures and subjective effects, some significant race effects were found such that measures associated with nicotine exposure (e.g. heart rate, dizzy, nauseous, headache, sick) increased significantly between bouts or time points for non-white but not white participants. Importantly, significant differences were also consistently observed between race groups. For example, reported ratings of "sick," "dizzy," and "lightheaded" were significantly higher for non-White participants than for White participants at various time-points. These findings suggest that non-White participants may be more sensitive to some of the aversive effects of nicotine commonly perceived by tobacco-naïve individuals.

This finding might be explained by work demonstrating race differences in smoking patterns and nicotine metabolism (St. Helen, Dempsey, Wilson, Jacob \& Benowitz, 2013). Racial differences in nicotine metabolism, in particular, have been attributed to the activity of the liver enzyme CYP2A6, which is responsible for conversion of nicotine into its metabolite, cotinine 
(National Center for Biotechnology Information, 2019). Specifically, gene variants (a.k.a. polymorphisms of the gene by the same name, CYP2A6) on chromosome 19 that code for the function of this enzyme have been shown to result in differences in nicotine metabolism (Oscarson et al., 1999; Nunoya et al., 1999; Yoshida et al., 2003) with varying prevalence rates between racial and ethnic groups (Nakajima et al., 2006; Schoedel, Hoffman, Rao, Sellers \& Tyndale, 2004). Specifically, Black/African American and Asian individuals have each been found to have a higher proportion of genetic polymorphisms associated with decreased nicotine metabolism (Nakajima et al., 2006; Schoedel, Hoffman, Rao, Sellers \& Tyndale, 2004; Tanner \& Tyndale, 2017), and to demonstrate a slower elimination rate than White individuals when they consume nicotine (Benowitz, Perez-Sable, Herrera \& Jacob, 2002; Ross et al., 2016). In cigarette smokers, the rate of nicotine metabolism is associated positively with the rewarding effects of nicotine. For example, fast nicotine metabolizers self-report more positive nicotine effects (i.e., "good effects," "like the drug effects," "want more drug") than slower metabolizers following a period of overnight tobacco abstinence (Sofuoglu, Herman, Nadim \& Jatlow, 2012). On the other hand, some studies suggest that relatively slow nicotine metabolism is associated with greater sensitivity to the rewarding nicotine effects (i.e., more time spent in a nicotine-associated compartment in a conditioned place preference test in mice; Li et al., 2013; also see Karp, O’Loughlin, Hanley, Tyndale \& Paradis, 2006 and O'Loughlin et al., 2004).

Though the pattern of findings from the present study seem to support the idea that racial minorities may experience significantly stronger aversive nicotine effects, only $9 \%$ of all possible race effects were significant. Importantly, some of these findings may alternatively be explained by differences in flavor-order; as 4 of the 5 non-White participants received the tobacco flavored ECIG during the first study session. Indeed, for all participants, ratings of 
nicotine effects (e.g., dizzy, lightheaded, nauseous) were significantly during the first compared to the second study session. Thus, differences in flavor ratings across race groups may be an artifact of the flavor received at session 1 rather than differences in metabolism. Additionally, while not statistically significant, mean lifetime ECIG use was lower for non-White participants $(M=10.5, S D=5.8)$ compared to White participants $(M=12.8, S D=10.8)$. Given the very small number of non-white individuals in the present sample $(n=5)$, analyses may not have been adequately powered to detect significant differences in lifetime use between groups; however, in a more diverse sample this pattern may better explain the observed differences in outcomes between race groups. As such, exploration of this pattern in a large-scale study is critical in order to fully understand these findings.

\section{Strengths}

There are several components of the present study which underlie its contribution to the existing literature on the abuse liability of flavoring in tobacco naïve young adults. Firstly, no other work has experimentally examined flavor preference in this population. In addition, the use of a within-subjects design increases confidence that findings are due to condition differences rather than due to between group variability. The combination of both subjective and behavioral outcomes also allowed the researchers to assess both perceptions of ECIG flavors, as well as their effects on behavior. Finally, given its current popularity both across ECIG users and among young adults, use of the JUUL device makes the results of this study particularly relevant to the national discussion regarding the regulation of ECIG products and strategies for preventing initiation by tobacco-naïve youth and young adults.

\section{Limitations}


Despite these study strengths, there are a variety of factors which may explain the overall paucity of significant results and limit the generalizability of these. The first limitation is that the majority of participants had very low levels of lifetime ECIG use $(\leq 10)$. These individuals may be representative of a certain sub-category of naïve users who are willing to sample the product but who do not intend to initiate regular product use. However, it is possible that non-users with higher lifetime use $(<100$ but $>50)$ may exhibit different patterns across outcomes. Naïve users with low levels of lifetime use appear to dislike using the electronic cigarette, as evidenced by overall low subjective ratings of product liking, lack of responding during the purchase task, and self-reports during the exit interview. For example, when comparing the five participants with the highest lifetime use (20 - 50 lifetime uses), reported mean scores ranging for ratings of product enjoyment (i.e., satisfying, pleasant, taste good) ranged from 26.8 to 31.4 ( $S D=26.8-$ 27.6). Comparatively, ratings for the five participants with the lowest lifetime use (3 to 4 lifetime uses) ranged from 22.3 to $25.4(S D=24.3-26.5)$. While these differences are relatively small, the effect might be magnified in a larger sample including more experience ECIG users. Future research should include non-users with a wider range of lifetime use in order to assess for differences in flavor preference and corresponding behavior based on ECIG experience.

The exit interview revealed that, while all of the participants had used an ECIG on more than one occasion, only six of the 30 participants reported product enjoyment as a reason for use. Moreover, these six individuals had varied number of lifetime ECIG uses: 3 lifetime uses $(n=1)$, 10 lifetime uses $(n=2), 15$ lifetime uses $(n=1)$, and 20 lifetime uses $(n=1)$. This may be suggestive in differing motivations for use among ECIG naïve users with low lifetime use. Indeed, throughout exit interviews, all participants described using the product in social settings. 
Notably, none of the participants used ECIGs without a peer present. Further, reasons such as "to fit in" and "peer pressure," were given for product initiation and continued experimentation despite $20 \%$ of participants reporting not liking the flavor of any ECIG that they had tried. Many participants also reported using the ECIG concurrently with alcohol, in settings such as parties, bars, the lake, or friends' houses. This is consistent with prior work regarding self-reported reasons for ECIG experimentation among young adults, which found that peer influence was one of the top three reasons for experimentation (Kong, Morean, Cavallo, Camenga, \& KrishnanSarin, 2015). In particular, young adults in the Kong et al. (2015) study reported being offered an ECIG by a friend or family member as a common reason for experimentation. Given the social nature of ECIG use among this sample, it is likely that the laboratory environment was not conducive to the enjoyment of the product.

Further, puffing behavior during directed bouts may have been influenced by the researcher's presence and effects of social desirability. While the majority of participants reported low ratings of product enjoyment (e.g., mean ratings of "pleasant," "satisfying," "taste good" = 18.9 - 22.5, $S E=2.3-3.4)$, average puff duration $(M=2.4$ seconds, $S E=0.14)$ was consistent with that recorded in prior work with ECIG-naïve adult cigarette smokers $(M=2.3$ seconds, $S D=0.2$ seconds; Farsalinos et al., 2014), though lower than that recorded in work with adult experienced ECIG users $(M=3.5$ seconds, $S D=0.2-1.4$ seconds; Farsalinos et al., 2015; St. Helen et al., 2017). Thus, despite self-reported dislike of the product, participants may have taken longer puffs than usual based on these demand characteristics.

In addition to challenges which may have been posed by a laboratory setting, aspects of the study design may also have affected participant behavior. The current study used a standard 10puff directed bout in which participants were instructed to puff from the ECIG every 30 -seconds, 
for 5-minutes. This bout structure has commonly been used in research using combustible cigarettes and older ECIG models (Spindle et al., 2015a; Vansickel et al., 2010; Vansickel \& Eissenberg, 2013). However, recent work suggests that, when allowed to smoke ad libitum, even experienced ECIG users do not puff in this pattern (Felicione, 2019; St. Helen et al., 2016). In one study, ECIG puffs were clustered into those considered "small” (2 - 5 puffs), "medium" (6 10 puffs), or "large" ( $>10$ puffs) assuming that the time in between those puffs was $\leq 60$ seconds while single puffs were those with $>60$ seconds separating them from puffs before and after (St. Helen et al., 2016). When experienced ECIG users puffed on their own device (cartridge-style, tank-style, or mod-style) ad libitum for 90 minutes, the majority were classified as "small" (43\%) compared to "medium" (28\%), "large" (17\%), or single (12\%) (St. Helen, et al., 2016). In work conducted in our laboratory (Felicione, 2019), ECIG-experienced users who puffed on their usual ECIG device (64\% of which were JUUL) demonstrated 49.4\% "small," 7.8\% "medium," $0.0 \%$ "large," and $41.9 \%$ single puffs. These results are consistent with the abovementioned study (St. Helen et al 2016) in that the proportion of small clustered puffs was greater than medium and large clustered puffs. On the other hand, our study demonstrated a notably high proportion of single puffs, as well as fewer medium and large puffs. (Felicione, 2019). This preference for shorter puffing bouts may be due to the higher nicotine concentration found in liquid for pod-mod style ECIG devices, relative to older generations. For example, , JUUL liquid contains $59 \mathrm{mg} / \mathrm{ml}$. In fact, when tested, some JUUL pods have been found to contain nicotine concentrations as high as $75 \mathrm{mg} / \mathrm{ml}$ (Eissenberg et. al, 2018; Talih et. al, 2019). However, a study comparing plasma nicotine concentration in adult smokers following directed 10-puff bouts with either JUUL or own-brand combustible cigarettes found that plasma levels were lower following JUUL use $(7.1 \mathrm{ng} / \mathrm{ml})$ than following cigarette use $(22.5 \mathrm{ng} / \mathrm{ml})$ (Maloney et al., 2019b). In light 
of these findings, it is likely that the standard 10-puff bout may be less appropriate for modeling naturalistic ECIG-use among pod-mod users, although this difference is not necessarily explained by differences in nicotine delivery. For tobacco naïve young adults, however, this amount does appear to exceed that which they would normally consume. This is reflected in exit interview reports, in which $73 \%$ of participants noted that the amount of ECIG liquid consumed in the session was more than what they would normally consume. Given these findings, development of a more appropriate standard puffing bout for newer generations of ECIGS may be necessary.

The study design may have also been limited by the lack of variety of ECIG flavor options for participants. While at the time of study development, fruit flavors were particularly popular (Ford et al., 2016; Soneji, 2019; Wang et al., 2015), flavor preference among young adults may also include mint, dessert, beverage and candy flavors. However, the majority of study participants reported preferring mint/menthol flavor $(n=10)$. Of note, JUUL pulled flavors other than mint and tobacco from the market during the course of the present study, which may have influenced this shift in self-reported preference. Indeed, recently published data collected in 2018 suggests that JUUL users were more likely to use the product with a mint/menthol flavor than users of other ECIG devices (Weaver et al., 2019). Given the shifting landscape of flavor regulations and trends, future work might include more flavor options in order to account for this and the heterogeneity of flavor preferences in this population.

Finally, due to the large number of analyses conducted (See Tables 2-5), the potential for experiment-wise Type I error rate for these results may be inflated. This number of analyses was utilized in order to fully investigate all potential effects in the present sample; however, results must be interpreted cautiously. Thus, significant effects should be interpreted with respect 
to effect size, which is less sensitive to Type I error inflation (Ranganathan, Pramesh, \& Buyse, 2016; Sullivan \& Feinn, 2012). In the present study, partial eta squared values were reported, which reflect the proportion of total variance in a particular outcome which is associated with the independent variable. Effect sizes for significant differences were small to medium (partial $\eta 2=$ 0.14 to 0.61 ), with larger effect sizes reflecting higher potential for reliability of an effect. Future work may consider recruiting a larger sample in order to increase study power or reducing the number of comparisons made to preserve the Type I error rate. Cautious interpretation of results and an emphasis on effect size will also aid in appropriate interpretation of results.

\section{Conclusions and Future Directions}

Results from the current study suggest that tobacco-naïve young adults with low levels of lifetime ECIG use may not respond differently to tobacco and fruit JUUL flavors. However, participants reported finding the device significantly more pleasant after the second compared to the first puffing bout in each session and reported lower levels of nicotine-driven subjective effects at the second compared to the first study session, indicating potential habituation to nicotine effects in a relatively short period of time. Further, the population identified in the present sample may be unique in their willingness to use ECIG products in social settings but without a desire to work for or use the product outside of these settings. A potential protective factor among these individuals may be their reported higher negative outcome expectancies compared to positive outcomes expectancies related to ECIG use. Expectancy ratings were highest for negative ECIG outcomes such as "hurt lungs" $(M=6.97, S D=2.1)$ and "become addicted" $(M=6.7, S D=2.6)$, and lowest for outcomes such as "become more popular" $(M=$ $2.3, S D=2.5)$ and "smell good" $(M=2.7, S D=2.5)$. Regarding risk perception, the majority of participants $(73.3 \%)$ reported agreeing with the statement "electronic cigarettes can help people 
quit smoking" and 50\% agreed with the statement "electronic cigarettes are less harmful than regular cigarettes". However, only $13.3 \%$ of participants agreed with the statement "electronic cigarettes are less harmful than regular cigarettes." Taken together, this suggests that despite reported social motivations for product use, this population of young adults perceives greater costs than benefit from ECIG use. While they believe that ECIGs may represent a helpful quit aid, the majority of participants did perceive risk of addiction associated with use. These expectancies and beliefs may underlie reported disinterest within this sample in engaging in regular ECIG use, despite willingness to sample the product in a social context. Future work should assess these outcomes across a wider range of non-users in order to clarify whether these same patterns hold for non-users with higher levels of lifetime ECIG use. Further, given changes in device styles and flavor popularity changes to future study designs should include lower puffing requirements during directed bouts and choice of a wider range of product flavors. This research is the first to experimental assess abuse liability of flavor among a sample of tobacco naïve young adults, and results suggest that among naïve users with low lifetime use there may be factors other than flavor which motivate product use and susceptibility. 


\section{References}

Agrawal, A. \& Lynskey, M.T. (2009). Tobacco and cannabis co-occurrence: Does rout of administration matter? Drug and Alcohol Dependence, 99: 240-247. doi: 10.1016/j.drugalcdep.2008.08.007

Ambrose, B. K., Day, H. R., \& Rostron, B. (2015). Flavored tobacco product use among US youth aged 12-17 years, 2013-2014. JAMA, 314, 1871-1873. doi:10.1001/jama.2015.13802

Amlung, M., Yurasek, A., McCarty, K. N., MacKillop, J., \& Murphy, J. G. (2015). Area under the curve as a novel metric of behavioral economic demand for alcohol. Experimental Clinical Psychopharmacology, 23, 168-175. doi: 10.1037/pha0000014

Audrain, McGovern, J. A., Strasser, A. A., \& Wileyto, E. P. (2016). The impact of flavoring on the rewarding and reinforcing value of e-cigarettes with nicotine among young adult smokers. Drug and Alcohol Dependence, 166, 263-267.

doi: 10.1016/j.drugalcdep.2016.06.030.

Aung, A. T., Pickworth, W. B., \& Moolchan, E. T. (2004). History of marijuana use and tobacco smoking topography in tobacco-dependent adolescents. Addictive Behavior, 29, 699-706. doi: 10.1016/j.addbeh.2004.02.012

Barnes, A. J., Bono, R. S., Lester, R. C., Eissenberg, T. E., \& Cobb, C. O. (2017). Effect of flavors and modified risk messages on e-cigarette abuse liability. Tobacco Regulatory Science, 3, 374-387. doi: 10.18001/TRS.3.4.1.

Barrett, S. P. (2010). The effects of nicotine, denicotinized tobacco, and nicotine containing tobacco on cigarette craving, withdrawal, and self-administration in male and female smokers. Behavioural Pharmacology, 21, 144-152. 
doi: 10.1097/FBP.0b013e328337be68

Benowitz, N. L., Herrera, B., \& Jacob, P. (2004). Mentholated cigarette smoking inhibits nicotine metabolism. Journal of Pharmacology and Experimental Therapeutics, 310, 120815. doi:10.1124/jpet.104.066902.

Benowitz, N. L., Perez-Stable, E. J., Herrera, B., \& Jacob, P. (2002). Slower metabolism and reduced intake of nicotine from cigarette smoking in Chinese-Americans. Journal of the National Cancer Institute, 94,108-115. doi: 10.1093/jnci/94.2.108

Benowitz, N. L., Jacob, P., Bernert, J. T., Wilson, M., Wang, L., Allen, F., \& Dempsey, D. (2005). Carcinogen exposure during short-term switching from regular to "light" cigarettes. Cancer Epidemiology Biomarkers \& Prevention, 14, 1376-1383. doi:10.1158/1055-9965.EPI-04-0667

Bernat, D., Gasquet, N., O’Dare Wilson, K., Porter, L., \& Choi, K. (2018). Electronic cigarette harm and benefit perceptions and use among youth. American Journal of Preventive Medicine, 55, 361 - 367. doi: 10.1016/j.amepre.2018.04.043.

Blank, M. D., Disharoon, S., \& Eissenberg, T. (2009). Comparison of methods for measurement of smoking behavior: mouthpiece-based computerized devices versus direct observation. Nicotine \& Tobacco Research, 11, 896-903. doi:10.1093/ntr/ntp083.

Blank, M. D., Cobb, C. O., Kilgalen, B., Austin, J., Weaver, M. F., Shihadeh, A., \& Eissenberg, T. (2011). Acute effects of waterpipe tobacco smoking: a double-blind, placebo-control study. Drug and Alcohol Dependence, 116, 102-109. doi:10.1015//j.drugalcdep.2010.11.026.

Bondy, S. J., Victor, J. C., \& Diemert, L. M. (2009). Origin and use of the 100 cigarette criterion in tobacco surveys. Tobacco Control, 18, 317-323. doi: 10.1136/tc.2008.027276 
Breland, A. B., Buchhalter, A. R., Evans, S. E., \& Eissenberg, T. (2002). Evaluating acute effects of potential reduced-exposure products for smokers: clinical laboratory methodology. Nicotine \& Tobacco Research, 4, S131-S140. doi:10.1080/1462220021000032780.

Brown and Williamson (1984). Taste segmentation study final report. Bates no. 538003902538003933, tobaccodocuments.org/bw/164129.html.

Brown, C. J., \& Cheng, J. M. (2014). Electronic cigarettes: product characterization and design considerations. Tobacco Control, 23, ii4-ii10. doi:10.1136/tobaccocontrol-2013-051476.

Caggiula, A. R., Donny, E. C., White, A. R., Chaudhri, N., Booth, S., \& Gharib, M. A. (2002). Environmental stimuli promote the acquisition of nicotine self-administration in rats. Psychopharmacology, 163, 230 - 237. doi: 10.1007/s00213-002-1156-5.

Carpenter, C. M., Wayne, G. F., Pauly, J. L., Koh, H. K. \& Connolly, G. (2005). New cigarette brands with flavors that appeal to youth: Tobacco marketing strategies. Journal of Health Affairs, 24, 1601-1610. doi: 10.1377/hlthaff.24.6.1601

Carpenter C. M., Connolly, G. N., Ayo-Yusuf, O. A. \& Wayne, G. F. (2009). Developing smokeless tobacco products for smokers: an examination of tobacco industry documents. Tobacco Control, 18, 54-59. doi: 10.1136/tc.2008.026583

Cassidy, S. (2011, October 26). How electronic cigarettes work. Retrieved April, 2018, from http://science.howstuffworks.com/innovation/everyday-innovations/electroniccigarette.htm.

Centers for Disease Control. (2017). National Health Interview Survey: Glossary[fact sheet]. Retrieved April, 2018 from: https://www.cdc.gov/nchs/nhis/tobacco/tobacco_glossary.htm

Chaffee, B. W., Gansky, S., Halpern-Felsher, B., Couch, E. T., Essex, G., Walsh, M. W. (2015). 
Conditional risk assessment of adolescents' electronic cigarette perceptions. American Journal of Health Behavior, 39, 421-432. doi: 10.5993/ajhb.39.3.14

Chaudhri, N., Caggiula, A. R., Donney, E. C., Palmatier, M. I. \& Sved, A. F. (2006). Complex interactions between nicotine and nonpharmacological stimuli reveal multiple roles for nicotine in reinforcement. Psychopharmacology, 184, 353-366.

doi: 10.1007/s00213-005-0178-1.

Choi, K., \&. Forster, J. L. (2014). Beliefs and experimentation with electronic cigarettes: A prospective analysis among young adults. American Journal of Preventive Medicine, 46, 175-178. doi: 10.1016/j.ampre.2013.0.007.

Cobb, C. O., Lopez, A. A., Soule, E. K., Yen, M. S., Rumsey, H., Lester Scholtes, R., Rudy, A. K., Lipato, T., Guy, M., \& Eissenberg, T. (2019). Influence of electronic cigarette liquid flavors and nicotine concentrations on subjective measures of abuse liability in young adult cigarette smokers. Drug and Alcohol Dependence, 203, 27 - 34. doi:

10.1016/j.drugalcdep.2019.05.024

Cobb, C. O., Weaver, M. F., \& Eissenberg, T. (2010). Evaluating the acute effects of oral, noncombustible reduced exposure products marketed to smokers. Tobacco Control, 19, 367373. doi:10.1136/tc2008.028993.

Cohen, J. (1992). A power primer. Psychological Bulletin, 112: 155-159. doi: 10.1037/00332909.112.1.155.

Cohn, A. M., Rose, S. W., Ilakkuvan, V., Gray, T., Curry, L., Villanti, A. C., Mays, D., Lindblom, E., Tercyak, K., Debnam, C., Mayo, A., \& Perreras, L. (2018). Harm perceptions of menthol and nonmenthol cigarettes differ by brand, race/ethnicity, and 
gender in US adult smokers: results from PATH Wave 1. Nicotine \& Tobacco Research, 21, 439-449. Doi: 10.1093/ntr/ntx277.

Connolly, G. (1995). The marketing of nicotine addiction by one oral snuff manufacturer. Tobacco Control, 4: 73-79.

Copp, S. R., Collins, J. L., Dar., R., \& Barrett, S. P. (2015). The effects of nicotine stimulus and response expectancies on male and female smokers' responses to nicotine-free electronic cigarettes. Addictive Behaviors, 40, 144-147. Doi: 10.1016/j.addbeh.2014.09.013

Cullen, K. A., Ambrose, B. K., Gentzke, A. S., Apelberg, B. J., Jamal, A., \& King. B.A. (2018). Notes from the field: Use of electronic cigarettes and any tobacco product among middle and high school students - United States, 2011 - 2018. MMWR Morbidity and Mortality Weekly Report, 1276 - 1277. Doi: 10.15585/mmwr.mm6745a5

Cummings, K. M., Morley, C. P., Horan, J. K., Steger, C., \& Leavel, N. R. (2002). Marketing to America's youth: Evidence from corporate documents. Tobacco Control, 11, i5-i17. Doi: 10.1136/tc.11.suppl_1.i5.

Dai, H., \& Hao, J. (2016). Flavored electronic cigarette use and smoking among youth. Pediatrics, 138. Doi:10.1542/peds.2016-2513

Dai, H., \& Leventhal, A. M. (2019). Prevalence of e-cigarette use among adults in the united states, 2014 - 2018 [Research Letter]. JAMA. doi: 10.1001/jama.2019.15331

Davis, R. M. (1987). Current trends in cigarette advertising and marketing. New England Journal of Medicine, 316, 725-732.

Etter, J. F. (2012). The electronic cigarette: an alternative to tobacco?. Jean-Francois Etter, Geneva, Switzerland.

Eissenberg T. (2014) Acute effects of electronic cigarettes in adults: nicotine delivery and abuse liability. In S. Krishnan-Sarin (Chair), Electronic Cigarettes: current state of the 
knowledge and future research. Symposium conducted at the 20th Annual Meeting of the Society for Research on Nicotine and Tobacco, Seattle, WA, February.

Eissenberg, T., Griffiths, R. R., \& Stitzer, M. L. (1996). Mecamylamine does not precipitate withdrawal in cigarette smokers. Psychopharmacology, 127, 328-336.

Eissenberg T, Soule E, Saliba N., et al. (2018). JUUL: The prototypical "pod mod": Design characteristics, toxicant yield, and preliminary nicotine delivery and subjective effect profile. Presented at the National Institutes of Health (NIH) Tobacco Regulatory Science Meeting, Bethesda, MD.

Evans, S. E., Blank, M., Sams, C., Weaver, M. F., \& Eissenberg, T. (2006). Transdermal nicotine-induced tobacco abstinence symptom suppression: nicotine dose and smokers' gender. Experimental and Clinical Psychopharmacology, 14 121-135.

doi: $10.1037 / 1064-1297.14 .2 .121$

Farsalinos, K. E. \& Polosa, R. (2014). Safety evaluation and risk assessment of electronic cigarettes as tobacco cigarette substitutes: A systematic review. Therapeutic Advances in Drug Safety, 5, 67-86. doi: 10.1177/2042098614524430

Farsalinos, K. E., Spyrou, A., Stefopoulos, C., Tsimopoulou, K., Kourkoveli, P., Tsiapras, D., Kyrzopoulos, S., Poulas, K., \& Voudris, V. (2015). Nicotine absorption from electronic cigarette use: comparison between experienced consumers (vapers) and naïve users (smokers). Scientific Reports, 5, 4133. doi:10.1038/srep011269.

Farsalinos, K. E., Spyrou, A., Tsimopoulou, K., Stefopoulos, C., Romagna, G., \& Voudris, V. (2014). Nicotine absorption from electronic cigarette use: comparison between first and new-generation devices. Scientific Reports, 4, 4133. doi:10.1038/srep04133.

Federal Trade Commission. (2017). Federal Trade Commission Cigarette Report for 
2015. Washington, DC: US Federal Trade Commission. Retrieved April, 2018 from: https://www.ftc.gov/system/files/documents/reports/federal-trade-commission-cigarettereport-2015-federal-trade-commission-smokeless-tobaccoreport/2015_cigarette_report.pdf

Felicione, N. (2019). Short-term abstinence effects in experienced electronic cigarette users (Unpublished doctoral dissertation). West Virginia University, Morgantown, WV.

Felicione, N. (2016). Comparison of Methods of Measuring Electronic Cigarette Puff Topography (Unpublished master's thesis). West Virginia University, Morgantown, WV.

Fielder, R. L., Carey, K. B. \& Carey, M. P. (2013). Hookah, cigarette, and marijuana use: A prospective study of smoking behaviors among first-year college women. Addictive Behaviors 38, 2729-2735. doi: 10.1016/j.addbeh.2013.07.006

FDA Regulation of Electronic Nicotine Delivery Systems and Investigation of Vaping Illnesses: Hearing before the House Committee on Energy and Commerce Subcommittee on Oversight and Investigations. (2019). (Testimony of Norman E. Sharpless)

Food and Drug Administration Deeming Tobacco Products to be Subject to the Federal Food, Drug, and Cosmetic Act. 81 Fed. Reg. 28974

Food and Drug Administration. (2017, July 28). FDA announces comprehensive regulatory plan to shift trajectory of tobacco-related disease, death [News release]. Retrieved October, 2019 from https://www.fda.gov/NewsEvents/Newsroom/PressAnnouncements/ucm568923.htm Food and Drug Administration. (2018). Vaporizers, e-cigarettes and other electronic nicotine 
delivery systems [fact sheet]. Retrieved October, 2019 from:

https://www.fda.gov/TobaccoProducts/Labeling/ProductsIngredientsComponents/ucm45 6610.htm

Ford, A., Mackintosh, A. M., Bauld, L., Moodie, C., \& Hastings, G. (2016). Adolescents' responses to the promotion and flavouring of e-cigarettes. International Journal of Public Health, 61, 215-224. doi: 10.1007/s00038-015-0769-5.

Gardiner, P. S. (2003). The African-Americanization of menthol cigarette use in the United States. Nicotine and Tobacco Research, 6, S55-S65. doi:

10.1080114622200310001649478.

Gentzke, A. S., Creamer, M., \& Cullen, K. A. (2019). Vital signs: Tobacco product use among middle and high school students - United States, 2011 - 2018. MMWR Morbidity and Mortality Weekly Report, 1276 - 1277. doi: 10.15585/mmwr.mm6745a5

Goldenson, N. I., Kirkpatrick, M. J., Barrington-Trimis, J. L., Pang, R. D, Mcbeth, J. F., Pentz, M. A., ... \& Leventhal, A. M. (2016). Effects of sweet flavorings and nicotine on the appeal and sensory properties of e-cigarettes among young adult vapers: Application of a novel methodology. Drug and Alcohol Dependence, 168, 176-180.

doi: 10.1016/j.drugalcdep.2016.09.014.

Greenwald, M. K. (2008). Behavioral economic analysis of drug preference using multiple choice procedure data. Drug and Alcohol Dependence, 93, 103-110. doi: 10.1016/j.drugalcdep.2007.09.002

Hiler, M. Z., Breland, A., Spindle, T., Maloney, S., Lipato, T., Shihadeh, A., Karaoghlanian, N., Lopez, A., Ramoa, C., \& Eissenberg, T. (2017). Electronic cigarette user plasma nicotine concentration, puff topography, heart rate, and subjective effects: Influence of liquid 
nicotine concentration and user experience. Experimental and Clinical Psychopharmocology, 25,: 380-392.

H.R. 1256 - 111 th Congress: Family Smoking and Prevention and Tobacco Control Act.

Retrieved April, 2018 from https://www.govtrack.us/congress/bills/111/hr1256

Jolma, C. D., Samson, R. A., Klewer, S. E., Donnerstein, R. L., \& Goldberg, S. J. (2002). Acute cardiac effects of nicotine in healthy young adults. Echocardiography, 19, 443-448.

Jones, A., Gulbis, A., \& Baker, E. H. (2010). Differences in tobacco use between Canada and the Unites States. International Journal of Public Health, 55, 167-175. doi: 0.1007/s00038-009-0101-3.

Karp, I., O’Loughlin, J., Hanley, J., Tyndale, R. F., \& Paradis, G. (2006) Risk factors for tobacco dependence in adolescent smokers. Tobacco Control, 15, 199-204.

doi: $10.1136 /$ tc. 2005.014118

Kim, H., Lim, J., Buehler, S. S., Brinkman, M. C., Johnson, N. M., Wilson, L., Cross, K. S., \& Clark, P. I. (2016). Role of sweet and other flavours in liking and disliking of electronic cigarettes. Tobacco Control, 25(s2): ii55-ii61. doi: 10.1136/tobaccocontrol-2016-053221.

King B. A., Dube, S. R., Tynan M. A.. (2013). Flavored cigar smoking among U.S. adults: findings from the 2009-2010 National Adult Tobacco Survey. Nicotine \& Tobacco Research, 15, 608-614. doi: 10.1093/ntr/nts178

Klein S. M., Giovino G. A., Barker D. C., Tworek C., Cummings K. M., \& O’Connor R. J. (2008). Use of flavored cigarettes among older adolescent and adult smokers: United States, 2004-2005. Nicotine \& Tobacco Research, 10, 1209-1214.

doi: 10.1080/14622200802163159 
Koffarnus, M. N., Franck, C. T., Stein, J. S., \& Bickel, W. K. (2015). A modified behavioral economic demand model to better describe consumption data. Experimental Clinical Psychopharmacology, 23, 504-512. doi: 10.1037/ph0000045

Kong, G., Morean, M.E., Cavallo, D.A., Camenga, D. R., \& Krishnan-Sarin, S. (2015). Reasons for electronic cigarette experimentation and discontinuation among adolescents and young adults. Nicotine \& Tobacco Research, 17, 847 - 854. doi:10.1093/ntr/ntu257.

Kosmider, L., Sobczak, A., Fik, M., Knysak, J., Zaciera, M., Kurek, J., \& Goniewicz, M. L. (2014). Carbonyl compounds in electronic cigarette vapors: Effects of nicotine solvent and battery output voltage. Nicotine \& Tobacco Research, 16, 1319-1326. doi:10.1093/ntr/ntu078.

Kostygina, G., Glantz, S. A. \& Ling, P. M. (2014). Tobacco industry use of flavours to recruit new users of little cigars and cigarillos. Tobacco Control. Published online first: 29 October 2014. doi: 10.1136/tobaccocontrol-2014-051830

Kostygina, G., \& Ling, P. M. (2016). Tobacco industry use of flavourings to promote smokeless tobacco products. Tobacco Control, 25, 1-10. doi:10.1136/tobaccocontrol-2015-053212

Kotecha, S., Jawad, M., \& Lliffe, S. (2016). Knowledge, attitudes and beliefs towards waterpipe tobacco smoking and electronic shisha (e-shisha) among young adults in London: A qualitative analysis. Primary Health Care Research and Development, 17, 166-174. doi: $10.1017 / \mathrm{S} 1463423615000237$

Kreslake J. M. \& Yerger V. B. (2010). Tobacco industry knowledge of the role of menthol in chemosensory perception of tobacco smoke. Nicotine and Tobacco Research, 12, S98101. doi:10.1093/ntr/ntq208

Krishnan-Sarin, S., Morean, M. E., Camenga, D. R., Cavallo, D., \& Kong, G. (2015). E-cigarette 
use among high school and middle school adolescents in Connecticut. Nicotine and Tobacco Research, 17, 810-818. doi: 10.1093/ntr/ntu243

Lechner, W. V., Meier, E., Wiener, J. L., Grant, D. M., Gilmore, J., Judah, M. R, Mills, A. C., \& Wagener, T. L. (2015). The comparative efficacy of first- versus second-generation electronic cigarettes in reducing symptoms of nicotine withdrawal. Addiction. 110, 862867. doi:10.1111/add.12870.

Leung, L. T., Ho, S. Y., Chen, J., Wang, M. P., \& Lam, T. H. (2017). Favourable perceptions of electronic cigarettes relative to cigarettes and the associations with susceptibility to electronic cigarette use in Hong Kong Chinese adolescents. International Journal of Environmental Research and Public Health, 15. doi: 10.3390/ijerph15010054.

Leventhal, A. M., Strong, D. R., Kirkpatrick, M. G., Unger, J. B., Sussman, S., Riggs, N. R., \& Audrain-McGovern, J. (2015). Association of electronic cigarette use with initiation of combustible tobacco product smoking in early adolescence. JAMA, 317, 700-707. doi: 10.1001/jama.2015.8950.

Li, L., Jia, K., Zhou, X., McCallum, S. E., Hough, L. B., \& Ding, X (2013). Impact of nicotine metabolism on nicotine's pharmacological effects and behavioral responses: Insights from a cyp2a(4/5)bgs-null mouse. Journal of Pharmacology and Experimental Therapeutics, 347, 746 - 754. doi: 10.1124/jpet.113.208256

Lipari, R. \& Jean-Francois, B. (2016). Trends in perception of risk and availability of substance use among full-time college students. Retrieved April, 2018 from: http://www.samhsa.gov/data/population-data-nsduh

Lui, T., David, S. P., Tyndale, R. F., Wang, H., Zhou, Q., Ding, P., He, Y. H., Yu, X. Q., Chen, 
W., Crump, C., Wen, X. Z., \& Chen, W. (2011). Associations of CYP2A6 genotype with smoking behaviors in southern China. Addiction, 106, 985 - 994. doi: 10.1111/j.13600443.2010 .03353

Maloney, S. A., Breland, A., Soule, E. K., Hiler, M., Ramoa, C., Lipato, T., \& Eissenberg, T. (2019a). Abuse liability assessment of an electronic cigarette in combustible cigarette smokers. Experimental Clinical Psychopharmacology, 27, 443 - 454.

doi: $10.1037 / \mathrm{pha} 0000261$

Maloney, S.A., Soule, E., Crabtree, M., Ratliff, L., Kilgalen, B., Lipato, T., Breland, A., \& Eissenberg, T. (2019b) Comparison of nicotine delivery and subjective effect profile of JUUL, IQOS, and combustible cigarettes. Poster presented at the 25th annual meeting of the Society for Research on Nicotine and Tobacco, San Francisco, CA.

McKelvey, K., Baiocchi, M., \& Halpern-Felsher, B. (2018). Adolescents' and young adults' use and perceptions of pod-based electronic cigarettes. JAMA, 1, e183535. doi:

10.1001/jamanetworkopen.2018.3535

Maziak, W., Ziyad Ben, T., Raed, B., Farahnaz, I., \& Rana, J. (2015). The global epidemiology of waterpipe smoking. Tobacco Control, 24, i3 - i12.

doi: 10.1136/tobaccocontrol-2014-051903

McDonald, E.A. \& Ling, P.M. (2015). One of several ‘toys’ for smoking: Young adult experiences with electronic cigarettes in New York City. Tobacco Control, 24, 588-593. doi: 10.1136/tobaccocontrol-2014-051743

McQueen, A., Tower, S., \& Sumner, W. (2011). Interviews with "vapers": Implications for future research with electronic cigarettes. Nicotine and Tobacco Research, 13, 860-867. doi: $10.1093 / n t r / n t r 088$. 
Nakajima M., Fukami, T., Yamanaka, H., Higashi, E., Sakai, H., Yoshida R., Kwon, J. T., McLeod, H. L., \& Yokoi, T. (2006). Comprehensive evaluation of variability in nicotine metabolism and CYP2A6 polymorphic alleles in four ethnic populations. Clinical Pharmacology \& Therapeutics, 80, 282-97. doi: 10.1016/j.clpt.2006.05.012

National Center for Biotechnology Information. (2019). CYP2A6. Gene ID: 1548. Retrieved October, 2019 from: https://www.ncbi.nlm.nih.gov/gene/1548

National Conference of State Legislatures. (2019). Alternative nicotine Products - Electronic cigarettes. Retrieved October, 2019 from: http://www.ncsl.org/research/health/alternative-nicotine-products-e-cigarettes.aspx National Institute on Drug Abuse. (2017). Young adults' daily use of marijuana a concern. Retrieved April, 2018 from: https://www.drugabuse.gov/news-events/newsreleases/2017/09/young-adults-daily-use-marijuana-concern

Nunoya, K. I., Yokoi, T., Kimura, K., Kainuma, T., Satoh, K., Kinoshita, M., \& Kamataki, T. (1999). A new CYP2A6 gene deletion responsible for the in vivo polymorphic metabolism of (+)-cis-3,5-dimethyl-2-(3-pyridyl)thiazolidin-4-one hydrochloride in humans. Journal of Pharmacology and Experimental Therapies, 289, 437-42.

O’Loughlin, J., Paradis, G., Kim, W., DiFranza, J., Meshefedjian, G., McMillan-Davey, E., Wong, S., Hanley, J., \& Tyndale, R. F. (2004). Genetically decreased CYP2A6 and the risk of tobacco dependence: a prospective study of novice smokers. Tobacco Control, 13, 422-428. doi: 10.1136/tc.2003.007070

Omvik, P. (1996). How smoking affects blood pressure. Blood Pressure, 5, 71-77.

Oscarson, M., McLellan, R. A., Gullsten, H., Yue, Q. Y. , Lang MA, Bernal, M. L., Sinues, B., 
Hirvonen, A., Rauino, H., Pelkonen, O., \& Ingelman-Sundberg, M. (1999)

Characterisation and PCR-based detection of a CYP2A6 gene deletion found at a high frequency in a Chinese population. FEBS Letters, 448,105-10. doi: 10.1016/s00145793(99)00359-2

Ozga, J. E., Felicione, N. J., Elswick, M. D., \& Blank, M.D. (2016). Acute effects of snus in never-tobacco users: a pilot study. The American Journal of Drug and Alcohol Abuse, 44, 113 - 119. doi: 10.1080/00952990.2016.1260581

Pacek, L., Kozink, R., Wimbish, C., \& McClernon, F. J. (2019). Appeal, subjective effects, and relative reinforcing effects of JUUL e-cigarettes that vary in flavor and nicotine content. Poster presented at the National Institute of Health Tobacco Regulatory Science Meeting, Bethesda, MD.

Perez-Stable, E. J., \& Benowitz, N. L. (2011). Do biological differences help explain tobaccorelated disparities? American Journal of Health Promotion, 25, s8-10. doi: 10.4278/ajhp.25.5.c2

Pesko, M. F., Kenkel, D. S., Wang, H. W., \& Hughers, J. M. (2016). The effect of potential electronic nicotine delivery system regulations on nicotine product selection. Addiction, 111, 734-744. doi: 10.1111/add.13257

Philip Morris. (1992). New flavors qualitative research insights. Bates no. 20231636982023163710, tobaccodocuments.org/pm/2023163698-3710.html.

Pokhrel, P., Lam, T. H., Pagano, I., Kawamoto, C. T., \& Herzog, T. A. (2018). Young adult ecigarette use outcome expectancies: Validity of a revised scale and a short scale. Addictive Behaviors, 78, 193-199. doi: 10.1016/j.addbeh.2017.11.019

Primack, B. A., Soneji, S., Stoolmiller, M., Fine, M. J., \& Sargent, J. D. (2015). Progression to 
traditional cigarette smoking after electronic cigarette use among U.S. adolescents and young adults. JAMA Pediatrics, 169, 1018-1023. doi: 10.1001/jamapediatrics.2015.1742

Ranganathan, P., Pramesh, C. S., \& Buyse, M. (2016). Common pitfalls in statistical analysis: The perils of multiple testing. Perspectives in Clinical Research, 7, 106-107. doi: $10.4103 / 2229-3485.179436$

R.J. Reynolds. (1988). Younger adult smoker opportunity. Bates no. 512466247-512466305, tobaccodocuments.org/rjr/512466247-6305.html.

Roma, P.G. Hursh, S.R., \& Hudja, S. (2016). Hypothetical purchase task questionnaires for behavioral economic assessments of value and motivation. Material and Decision Economics, 37, 306-323. doi: 10.1002/mde.2718

Rose, J. E., \& Behm, F. M. (2004). Extinguishing the rewarding value of smoke cues: Pharmacological and behavioral treatments. Nicotine \& Tobacco Research, 6, 523-532. doi: 10.1080/14622200410001696501.

Rose, S. W., Johnson, A. L., Glasser, A. M., Villanti, A. C., Ambrose, B. K., Conway, K. P., Cummings, K. M., Stanton, C. A., Delnevo, C., Wackowski, O.A., Edwards, K. C., Feirman, S. P., Bansal-Travers, M., Bernat, J., Holder-Hayes, E., Green, V., Silveira, M. L., Zhou, Y., Abudayyeh, H., \& Hyland, A. (2019). Flavor types used by youth and adult tobacco users in wave 2 of the population assessment of tobacco and health (PATH) study, 2014-15. Tobacco Control. Advance online publication. doi:

10.1136/tobaccocontrol-2018-054852.

Ross, K. C., Gubner, N. R., Tyndale, R. F., Hawk, L. W., Lerman, C., George, T. P., Cinciripinim P, Schnoll, R. A., \& Benowitz, N. L. (2016). Racial differences in the relationship between rate of nicotine metabolism and nicotine intake from cigarette 
smoking. Pharmacology, Biochemistry, and Behavior, 148, 1 - 7. doi:

10.1016/j.pbb.2016.05.002

Schoedel, K. A., Hoffmann, E. B., Rao, Y., Sellers, E. M., Tyndale, R. F. (2004). Ethnic variation in CYP2A6 and association of genetically slow nicotine metabolism and smoking in adult Caucasians. Pharmacogenetics, 14, 615-26. doi: 10.1097/00008571200409000-00006

Shihadeh, A., \& Eissenberg, T. (2015). Electronic cigarette effectiveness and abuse liability: predicting and regulating nicotine flux. Nicotine \& Tobacco Research, 17, 158-162. doi:10.1093/ntr/ntu175

Sofuoglu, M., Herman, A. I., Nadim, H., \& Jatlow, P. (2012). Rapid nicotine clearance is associated with greater reward and heart rate increases from intravenous nicotine. Neuropsychopharmacology, 37, 1509-1516. doi: 10.1038/npp.2011.336

Soneji, S. S., Knutzen, K. W., \& Villanti, A. C. (2019). Use of flavored e-cigarettes among adolescents, young adults, and older adults: Findings from the Population Assessment for Tobacco and Health Study. Public Health Reports, 134, 282 - 292.

doi: $10.1177 / 0033354919830967$

SRNT Committee on Biochemical Verification. (2002). Biochemical verification of tobacco use and cessation. Nicotine and Tobacco Research, 4, 149-159.

Spindle, T. R., Talih, S., Hiler, M. M., Karaoghlanian, N., Halquist, M. S., Breland, A. B., Shihdeh, A., \& Eissenberg, T. (2018). Effects of electronic cigarette liquid solvents propylene glycol and vegetable glycerin on user nicotine delivery, heart rate, subjective effects, and puff topography. Drug and Alcohol Dependence, 188, 193-199. doi: 10.1016/j.drugalcdep.2018.03.042 
Spindle, T. R., Breland, A. B., Karaoghlanian, N. V., Shihadeh, A. L., \& Eissenberg, T. (2015a). Preliminary results of an examination of electronic cigarette user puff topography: the effect of a mouthpiece-based topography measurement device on plasma nicotine and subjective effects. Nicotine \& Tobacco Research, 17, 142-149. doi:10.1093/ntr/ntu186

Spindle, T., Pettaway, K., Breland, A., Karaoghlanian, N., Shihadeh, A., \& Eissenberg, T. (2015b). Does electronic cigarette liquid nicotine concentration and user experience influence puff topography?. Poster presented at the 21 st annual meeting of the Society for Research on Nicotine and Tobacco, Philadelphia, PA, February.

St. Helen, G. (2018). Impact of e-liquid flavors on vaping behavior. Symposium presented at the 24th annual meeting of the Society for Research on Nicotine and Tobacco, Baltimore, MD.

St. Helen, G., Dempsey, D. A., Havel, C. M., Jacob, P. \& Benowitz, N. L. (2017). Impact of eliquid flavors on nicotine intake and pharmacology of e-cigarettes. Drug and Alcohol Dependence, 178, 391-398. doi: 10.1016/j.drugalcdep.2017.05.042

St. Helen, G., Dempsey, D. A., Wilson, M., Jacob, P., \& Benowitz, N. L. (2013). Racial differences in the relationship between tobacco dependence and nicotine and carcinogen exposure. Addiction, 108, 607 - 617. doi:10.1111/j.1360-0443.2012.04077

St. Helen, G., Ross, K. C., Dempsey, D. A., Havel, C. M., Jacob, P., \& Benowitz, N. L. (2016). Nicotine delivery and vaping behavior during ad libitum e-cigarette access. Tobacco Regulatory Science, 2: 363 - 376. doi:10.18001/TRS.2.4.8

Stein, J. S. \& Bickel, W. K. (2018). Initial validation of a probability-based purchase task to assess abuse liability of novel tobacco products. Poster presented at the $24_{\text {th }}$ annual meeting of the Society for Research on Nicotine and Tobacco, Baltimore, MD, February. 
Sullivan, G. M. \& Feinn, R. (2012). Using effect size - or why the p value is not enough. Journal of Graduate Medical Education, 4, 279 - 282. doi: 10.4300/JGME-D-12-00156.1.

Tackett, A. P., Lechner, W. V., Meier, E., \& Grant, D. M. (2015). Biochemically verified smoking cessation and vaping beliefs among vape store customers. Addiction, 110, 868874. doi:10.1002/add.12878

Talih, S., Balhas, Z., Eissenberg, T., Salman, R., Karaoghlanian, N., El Hallani, A., Baalbaki, R., Saliba, N., \& Shihadeh, A. (2015). Effects of user puff topography, device voltage, and liquid nicotine concentration on electronic cigarette nicotine yield: Measurements and model predictions. Nicotine \& Tobacco Research, 17, 150-157. doi:10.1093/ntr/ntu174

Talih, S., Salman, R., El-Hage, R., Karam, E., Karaoghlanian, N., El-Hellani, A., Saliba, N., \& Shihadeh, A. (2019). Characteristics and toxicant emissions of JUUL electronic cigarettes. Tobacco Control. Advance online publication. doi: 10.1136/tobaccocontrol2018-054616

Tanner, J. \& Tyndale, R. F. (2017). Variation in CYP2A6 activity and personalized medicine. Journal of Personalized Medicine, 7, 18. doi: 10.3390/jpm7040018

Tobacco Institute. Kentucky’s Tobacco Heritage. Brown \& Williamson Records. https://www.industrydocumentslibrary.ucsf.edu/tobacco/dosc/jrpf0146

Trehy, M. L., Ye, W., Hadwiger, M. E., Moore, T. W., Allgire, J. F., Woodruff, J. T., Ahadi, S. S., Black, J. C., \& Westenberger, B.J. (2011). Analysis of electronic cigarette cartridges, refill solutions, and smoke for nicotine and nicotine related impurities. Journal of Liquid Chromatography \& Related Technologies, 34, 1442-1458. doi:10.1080/10826076.2011.572213

Tsai, J., Walton, K., Coleman, B. N. Saida, R. S., Johnson, S. E., Kennedy, S. M., \& Caraballo, 
R. S. (2018). Reasons for electronic cigarette use among middle and high school students - National Youth Tobacco Survey, United States, 2016. Morbidity and Mortality Weekly Report, 67. doi:10.15585/mmwr.mm6706a5

U.S. Department of Health and Human Services, Public Health Service, Office of the Surgeon General. (2016). E-cigarette use among youth and young adults: A report of the Surgeon General. Rockville, MD.

U.S. Smokeless Tobacco Company, David Weiss Associates. (1984). The 'Graduation Theory'. Bates No. USSTC1945141-USSTC1945142. Retrieved April, 2018 from http://legacy.library.ucsf.edu/tid/lfc46b00

Vansickel, A. R., Cobb, C. O., Weaver, M. F., \& Eissenberg, T. E. (2010). A clinical laboratory model for evaluating the acute effects of electronic "cigarettes": Nicotine delivery profile and cardiovascular and subjective effects. Cancer Epidemiology, Biomarkers \& Prevention, 19, OF1-OF9. doi:10.1158/1055-9965.EPI-10-0288

Vansickel, A. R., \& Eissenberg, T. (2013). Electronic cigarettes: Effective nicotine delivery after acute administration. Nicotine \& Tobacco Research, 15, 267-270. doi:10.1093/ntr/ntr316

Villanti A. C., Richardson A., Vallone D. M., \& Rath J. M. (2013). Flavored tobacco product use among U.S. young adults. American Journal of Preventative Medicine, 44, 388-391. doi: 10.1016/j.amepre.2012.11.031

Villanti, A. C., Johnson, A. L., Ambrose, B. K., Cummings, K. M., Stanton, C. A., Rose, S. W. Feirman, S. P., Tworek, C., Glasser, A. M., Pearson, J. L., Cohn, A. M., Conway, K. P., Niaura, R. S., Bansal-Travers, M., \& Hyland, A. (2017). Flavored tobacco product use in youth and adults: Findings from the first wave of the PATH study. American Journal of Preventive Medicine, 53, 139-151. doi: 10.1016/j.ampre.2017.01.026 
Wagener, T. L., Floyd, E. L., Stepanov, I., Driskill, L. M., Frank, S. G., Meier, E., Leavens, E.

Walele, T., Sharma, G., Savioz, R., Martin, C., \& Williams, J. (2016). A randomized, crossover study on an electronic vapour product, a nicotine inhalator and a conventional cigarette. Part A: Pharmacokinetics. Regulatory Toxicology and Pharmacology, 74, 187-192. doi: 10.1016/j.yrtph.2015.12.003

Wang, L., Zhan, Y., Li, Q., Zeng, D., Lieschow, S. J., \& Okamoto, J. (2015). An examination of electronic cigarette content on social media: Analysis of e-cigarette flavor content on Reddit. International Journal of Environmental Research and Public Health, 12: 1493314952. doi: 10.3390/ijerph121114933

Weaver, S., Heath, J., Huang, J., Ashley, D., Pechacek, T., \& Eriksen, M. (2019). Sociodemographic and tobacco use characteristics of US adult users of the JUUL. Poster presented at the National Institute of Health Tobacco Regulatory Science Meeting, Bethesda, MD.

Wickham, R. J. (2015). How menthol alters tobacco-smoking behavior: A biological perspective. Yale Journal of Biological Medicine, 88(3): 279-87.

Wickham, R. J., Nunes, E.J., Hughley, S., Silva, P., Walton, S. N., Park, J., \& Addy, N. A. (2017). Evaluating oral flavorant effects on nicotine self-administration behavior and phasic dopamine signaling. Neuropharmacology, 128, 33-42.

doi: 10.1016/j.neuropharm.2017.09.029

Willner, P., \& Jones, C. (1996). Effects of mood manipulation on subjective and behavioural measures of cigarette craving. Behavioral Pharmacology, 6,1-9.

Willner, P., Hardman, S., \& Eaton, G. (1995). Subjective and behavioural evaluation of cigarette 
cravings. Psychopharmacology 118, 171-177

Wills, T. A., Knight, R., Sargent, J. D., Gibbons, F. X., Pagano, I., \& Williams, R. J. (2016). Longitudinal study of e-cigarette use and onset of cigarette smoking among high school students in Hawaii. Tobacco Control, 26, 34-39.

doi: 10.1136/tobaccocontrol-2015-052705

Yoshida, R., Nakajima, M., Nishimura, K., Tokudome, S., Kwon, J.T., \& Yokoi, T. (2003). Effects of polymorphism in promoter region of human CYP2A6 gene (CYP2A6*9) on expression level of messenger ribonucleic acid and enzymatic activity in vivo and in vitro. Clinical Pharmacology \& Therapeutics,74, 69-76. doi: 10.1016/S00099236(03)00090-0

Zacny, J. P. \& Stitzer, M.L. (1988). Cigarette brand-switching: effects on smoke exposure and smoking behavior. Journal of Pharmacology and Experimental Therapeutics, 246, 619627.

Zacny, J. P., Stitzer, M. L., Brown, F. J., Yingling, J. E., \& Griffiths, R. R. (1987). Human cigarette smoking: effects of puff and inhalation parameters on smoke exposure. Journal of Pharmacology and Experimental Therapeutics, 240, 554-564.

Zare, S., Nemati, M., \& Zheng, Y. (2018). A systematic review of consumer preference for ecigarette attributes: Flavor, nicotine strength, and type. PLoS One, 13, e0194145. doi: 10.1371/journal.pone.0194145

Zhu, S. H., Sun, J. Y., Bonnevie, E., Cummins, S. E., Gamst., A., Yin, L., \& Lee, M. (2014). Four hundred and sixty brands of e-cigarettes and counting: Implications for product regulation. Tobacco Control, 23, iii3-9. doi: 10.1136/tobaccocontrol-2014-051670 
Table 1 
Participant Baseline Characteristics

\begin{tabular}{|c|c|}
\hline & $M(S D)$ or $\%$ \\
\hline \multicolumn{2}{|l|}{ Gender } \\
\hline Male & $36.67 \%$ \\
\hline Female & $63.33 \%$ \\
\hline \multicolumn{2}{|l|}{ Race } \\
\hline Caucasian & $83.30 \%$ \\
\hline $\begin{array}{r}\text { Black/ African } \\
\text { American }\end{array}$ & $3.30 \%$ \\
\hline Asian & $3.30 \%$ \\
\hline$>1$ race & $10.00 \%$ \\
\hline \multicolumn{2}{|l|}{ Ethnicity } \\
\hline Non-Hispanic/Latino & $100 \%$ \\
\hline Age (Years) & $19.43(1.36)$ \\
\hline \multicolumn{2}{|l|}{ Employment } \\
\hline Student & $83.33 \%$ \\
\hline Full-time & $6.67 \%$ \\
\hline Part-time & $6.67 \%$ \\
\hline Unemployed & $3.33 \%$ \\
\hline Expired Air CO (ppm) & $2.13(1.61)$ \\
\hline Urinary Cotinine (ng/ml) & $1.13(0.35)$ \\
\hline \# Lifetime ECIG Uses & $12.37(10.12)$ \\
\hline \multicolumn{2}{|l|}{ Liquid Fruit Choice } \\
\hline Mango & $56.67 \%$ \\
\hline Fruit Medley & $43.33 \%$ \\
\hline
\end{tabular}

Table 2 


\section{Repeated Measure ANCOVA Results by Flavor}

\begin{tabular}{|c|c|c|c|c|c|c|c|c|c|c|c|c|c|c|c|c|c|c|c|c|c|}
\hline \multirow[b]{2}{*}{ Outcome Measure } & \multicolumn{3}{|c|}{ Flavor } & \multicolumn{3}{|c|}{ Bout } & \multicolumn{3}{|c|}{ Time } & \multicolumn{3}{|c|}{ Flavor x Bout } & \multicolumn{3}{|c|}{ Flavor $\mathrm{x}$ Time } & \multicolumn{3}{|c|}{ Bout $\mathrm{x}$ Time } & \multicolumn{3}{|c|}{$\begin{array}{c}\text { Flavor } \mathrm{x} \text { Bout } \mathrm{x} \\
\text { Time }\end{array}$} \\
\hline & $F$ & $p$ & $\eta_{\mathrm{p}}^{2}$ & $F$ & $p$ & $\eta_{p}^{2}$ & $F$ & $p$ & $\eta_{p}^{2}$ & $F$ & $p$ & $\eta_{p}^{2}$ & $F$ & $p$ & $\eta_{p}^{2}$ & $F$ & $p$ & $\eta_{\mathrm{p}}^{2}$ & $F$ & $p$ & $\eta_{\mathrm{p}}^{2}$ \\
\hline \multicolumn{22}{|l|}{ Physiological Measures $^{\mathrm{a}}$} \\
\hline Heart Rate & 0.47 & 0.500 & 0.02 & 2.69 & 0.113 & 0.09 & 7.44 & 0.011 & 0.22 & 0.64 & 0.430 & 0.02 & 0.67 & 0.419 & 0.03 & 1.03 & 0.320 & 0.04 & 0.43 & 0.516 & 0.02 \\
\hline Systolic & 5.34 & 0.029 & 0.17 & 0.15 & 0.299 & 0.04 & 3.00 & 0.095 & 0.10 & 0.22 & 0.645 & 0.01 & 0.07 & 0.792 & 0.00 & 6.46 & 0.017 & 0.20 & 1.51 & 0.230 & 0.06 \\
\hline Diastolic & 1.24 & 0.276 & 0.05 & 0.50 & 0.485 & 0.02 & 1.47 & 0.236 & 0.05 & 0.25 & 0.624 & 0.01 & 0.22 & 0.641 & 0.01 & 1.12 & 0.300 & 0.04 & 0.01 & 0.915 & 0.00 \\
\hline \multicolumn{22}{|l|}{ Direct Effects of Nicotine ${ }^{b}$} \\
\hline Dizzy & 0.48 & 0.494 & 0.02 & 0.42 & 0.524 & 0.02 & 0.13 & 0.724 & 0.01 & 1.32 & 0.261 & 0.05 & 4.85 & 0.037 & 0.16 & 0.14 & 0.715 & 0.01 & 0.03 & 0.860 & 0.00 \\
\hline Lightheaded & 2.39 & 0.135 & 0.09 & 0.08 & 0.787 & 0.00 & 0.39 & 0.540 & 0.02 & 0.02 & 0.890 & 0.00 & 2.94 & 0.099 & 0.11 & 0.30 & 0.592 & 0.01 & 0.08 & 0.784 & 0.00 \\
\hline Nauseaus & 0.69 & 0.415 & 0.03 & 0.30 & 0.590 & 0.01 & 0.10 & 0.754 & 0.00 & 2.72 & 0.112 & 0.10 & 0.26 & 0.616 & 0.01 & 2.51 & 0.126 & 0.09 & 0.02 & 0.878 & 0.00 \\
\hline Nervous & 0.66 & 0.424 & 0.03 & 0.76 & 0.390 & 0.03 & 0.22 & 0.640 & 0.01 & 0.04 & 0.844 & 0.00 & 0.31 & 0.584 & 0.01 & 1.01 & 0.325 & 0.04 & 0.01 & 0.929 & 0.00 \\
\hline Sweaty & 1.61 & 0.216 & 0.06 & 0.26 & 0.613 & 0.01 & 0.40 & 0.534 & 0.02 & 1.51 & 0.230 & 0.06 & 0.04 & 0.839 & 0.00 & 0.36 & 0.552 & 0.01 & 0.52 & 0.479 & 0.02 \\
\hline Headache & 0.08 & 0.785 & 0.00 & 0.01 & 0.931 & 0.00 & 0.46 & 0.505 & 0.02 & 1.71 & 0.203 & 0.06 & 0.04 & 0.846 & 0.00 & 0.01 & 0.916 & 0.00 & 0.01 & 0.908 & 0.00 \\
\hline Excess Salivation & 2.42 & 0.133 & 0.09 & 1.34 & 0.257 & 0.05 & 0.01 & 0.940 & 0.00 & 2.40 & 0.134 & 0.09 & 0.14 & 0.709 & 0.01 & 0.38 & 0.541 & 0.02 & 0.00 & 0.992 & 0.00 \\
\hline Heart Pounding & 1.27 & 0.270 & 0.05 & 0.64 & 0.430 & 0.03 & 0.00 & 0.975 & 0.00 & 0.15 & 0.706 & 0.01 & 0.14 & 0.710 & 0.01 & 0.00 & 0.988 & 0.00 & 0.77 & 0.388 & 0.03 \\
\hline Confused & 0.38 & 0.543 & 0.02 & 1.28 & 0.268 & 0.05 & 0.21 & 0.650 & 0.01 & 0.01 & 0.909 & 0.00 & 0.74 & 0.397 & 0.03 & 0.04 & 0.842 & 0.00 & 4.75 & 0.039 & 0.16 \\
\hline Weak & 0.04 & 0.839 & 0.00 & 0.00 & 0.986 & 0.00 & 0.04 & 0.839 & 0.00 & 1.62 & 0.215 & 0.06 & 0.00 & 0.958 & 0.00 & 0.02 & 0.882 & 0.00 & 0.05 & 0.832 & 0.00 \\
\hline \multicolumn{22}{|l|}{ Direct Effects of Product Use $\mathrm{b}^{\mathrm{b}}$} \\
\hline Satisfying & 0.01 & 0.938 & 0.00 & 2.45 & 0.130 & 0.09 & 0.00 & 0.974 & 0.00 & 2.15 & 0.155 & 0.08 & 0.05 & 0.819 & 0.00 & 1.18 & 0.287 & 0.05 & 0.35 & 0.561 & 0.01 \\
\hline Pleasant & 0.02 & 0.901 & 0.00 & 4.31 & 0.048 & 0.15 & 0.37 & 0.551 & 0.01 & 0.24 & 0.626 & 0.01 & 0.17 & 0.687 & 0.01 & 0.88 & 0.356 & 0.03 & 0.03 & 0.873 & 0.00 \\
\hline Taste Good & 0.34 & 0.563 & 0.01 & 4.99 & 0.030 & 0.17 & 0.97 & 0.334 & 0.04 & 0.00 & 0.983 & 0.00 & 0.25 & 0.622 & 0.01 & 6.31 & 0.019 & 0.20 & 4.08 & 0.054 & 0.14 \\
\hline Dizzy & 1.73 & 0.201 & 0.07 & 3.87 & 0.060 & 0.13 & 0.01 & 0.920 & 0.00 & 1.57 & 0.222 & 0.06 & 0.01 & 0.912 & 0.00 & 2.27 & 0.144 & 0.08 & 1.05 & 0.315 & 0.04 \\
\hline Calm & 1.31 & 0.263 & 0.05 & 0.59 & 0.452 & 0.02 & 1.16 & 0.291 & 0.04 & 2.54 & 0.124 & 0.09 & 0.04 & 0.840 & 0.00 & 5.45 & 0.028 & 0.18 & 0.06 & 0.815 & 0.00 \\
\hline Concentrate & 0.35 & 0.561 & 0.01 & 0.08 & 0.774 & 0.00 & 1.51 & 0.231 & 0.06 & 0.60 & 0.446 & 0.02 & 0.15 & 0.707 & 0.01 & 2.82 & 0.106 & 0.10 & 0.10 & 0.750 & 0.00 \\
\hline Awake & 0.03 & 0.854 & 0.00 & 1.20 & 0.283 & 0.05 & 0.48 & 0.494 & 0.02 & 1.96 & 0.174 & 0.07 & 0.22 & 0.643 & 0.01 & 0.07 & 0.801 & 0.00 & 0.03 & 0.855 & 0.00 \\
\hline Reduced Hunger & 0.05 & 0.827 & 0.00 & 0.80 & 0.381 & 0.03 & 0.00 & 0.960 & 0.00 & 0.00 & 0.970 & 0.00 & 0.37 & 0.550 & 0.01 & 2.93 & 0.099 & 0.11 & 0.08 & 0.783 & 0.00 \\
\hline Sick & 1.78 & 0.194 & 0.07 & 0.66 & 0.426 & 0.03 & 0.94 & 0.342 & 0.04 & 0.14 & 0.715 & 0.01 & 2.40 & 0.134 & 0.09 & 0.98 & 0.333 & 0.04 & 1.60 & 0.218 & 0.06 \\
\hline \multicolumn{22}{|l|}{ gLMS $^{\mathrm{c}}$} \\
\hline Sensation & 0.20 & 0.660 & 0.02 & 0.17 & 0.685 & 0.01 & & & & 0.08 & 0.789 & 0.01 & & & & & & & & & \\
\hline Harshness & 1.02 & 0.332 & 0.08 & 1.39 & 0.262 & 0.10 & & & & 1.84 & 0.200 & 0.13 & & & & & & & & & \\
\hline Throat Hit & 0.36 & 0.561 & 0.03 & 4.09 & 0.066 & 0.26 & & & & 0.38 & 0.547 & 0.03 & & & & & & & & & \\
\hline Puff Duration $^{\mathrm{b}}$ & 0.85 & 0.364 & 0.03 & 0.57 & 0.459 & 0.02 & & & & 0.73 & 0.400 & 0.03 & & & & & & & & & \\
\hline
\end{tabular}
${ }^{a} d f=1,23 ;{ }^{b} d f=1,25 ;{ }^{c} d f=1,12$

Table 3 


\section{Mixed Repeated-Measure ANOVA Results by Race}

\begin{tabular}{|c|c|c|c|c|c|c|c|c|c|c|c|c|c|c|c|c|c|c|c|c|c|}
\hline \multirow[b]{2}{*}{ Outcome Measure } & \multicolumn{3}{|c|}{ Flavor x Race } & \multicolumn{3}{|c|}{ Bout x Race } & \multicolumn{3}{|c|}{ Time $\mathrm{x}$ Race } & \multicolumn{3}{|c|}{$\begin{array}{c}\text { Flavor x Bout x } \\
\text { Race } \\
\end{array}$} & \multicolumn{3}{|c|}{$\begin{array}{c}\text { Flavor } \mathrm{x} \text { Time } \mathrm{x} \\
\text { Race } \\
\end{array}$} & \multicolumn{3}{|c|}{ Bout x Time x Race } & \multicolumn{3}{|c|}{$\begin{array}{c}\text { Flavor x Bout x Time } \\
\text { x Race } \\
\end{array}$} \\
\hline & $F$ & $p$ & $\eta_{\mathrm{p}}{ }^{2}$ & $F$ & $p$ & $\eta_{p}^{2}$ & $F$ & $p$ & $\eta_{\mathrm{p}}^{2}$ & $F$ & $p$ & $\eta_{\mathrm{p}}^{2}$ & $F$ & $p$ & $\eta_{\mathrm{p}}^{2}$ & $F$ & $p$ & $\eta_{p}^{2}$ & $F$ & $p$ & $\eta_{p}^{2}$ \\
\hline \multicolumn{22}{|l|}{ Physiological Measures $^{\mathrm{a}}$} \\
\hline Heart Rate & 0.53 & 0.474 & 0.02 & 0.04 & 0.850 & 0.00 & 1.58 & 0.220 & 0.06 & 0.76 & 0.374 & 0.55 & 1.80 & 0.190 & 0.07 & 11.05 & 0.003 & 0.30 & 11.20 & 0.002 & 0.30 \\
\hline Systolic & 0.32 & 0.575 & 0.01 & 0.00 & 0.997 & 0.00 & 0.22 & 0.650 & 0.01 & 0.88 & 0.358 & 0.03 & 2.24 & 0.147 & 0.08 & 1.40 & 0.248 & 0.25 & 4.65 & 0.041 & 0.15 \\
\hline Diastolic & 0.10 & 0.759 & 0.00 & 0.32 & 0.575 & 0.01 & 0.00 & 0.956 & 0.00 & 5.68 & 0.025 & 0.18 & 1.13 & 0.298 & 0.04 & 2.80 & 0.106 & 0.10 & 7.30 & 0.012 & 0.22 \\
\hline \multicolumn{22}{|l|}{ Direct Effects of Nicotine ${ }^{b}$} \\
\hline Dizzy & 0.32 & 0.579 & 0.01 & 3.70 & 0.065 & 0.12 & 6.25 & 0.019 & 0.18 & 0.29 & 0.595 & 0.01 & 2.22 & 0.147 & 0.07 & 0.67 & 0.420 & 0.02 & 0.00 & 0.961 & 0.00 \\
\hline Lightheaded & 0.64 & 0.430 & 0.02 & 1.30 & 0.264 & 0.04 & 1.28 & 0.268 & 0.04 & 1.36 & 0.253 & 0.05 & 5.25 & 0.030 & 0.16 & 0.23 & 0.639 & 0.01 & 0.62 & 0.439 & 0.02 \\
\hline Nauseaus & 0.06 & 0.808 & 0.00 & 3.79 & 0.062 & 0.12 & 0.50 & 0.486 & 0.02 & 5.67 & 0.024 & 0.17 & 1.18 & 0.288 & 0.04 & 0.07 & 0.801 & 0.00 & 1.20 & 0.284 & 0.04 \\
\hline Nervous & 1.31 & 0.262 & 0.05 & 0.01 & 0.945 & 0.00 & 0.39 & 0.538 & 0.01 & 2.64 & 0.115 & 0.09 & 0.70 & 0.411 & 0.02 & 0.01 & 0.910 & 0.00 & 0.00 & 0.955 & 0.00 \\
\hline Sweaty & 6.68 & 0.015 & 0.19 & 0.86 & 0.362 & 0.03 & 4.02 & 0.055 & 0.13 & 3.63 & 0.067 & 0.12 & 1.06 & 0.312 & 0.04 & 1.11 & 0.300 & 0.04 & 1.52 & 0.230 & 0.05 \\
\hline Headache & 0.01 & 0.913 & 0.00 & 6.06 & 0.020 & 0.18 & 1.36 & 0.253 & 0.05 & 0.01 & 0.937 & 0.00 & 0.01 & 0.938 & 0.00 & 4.92 & 0.035 & 0.15 & 0.84 & 0.367 & 0.03 \\
\hline Excess Salivation & 0.82 & 0.372 & 0.03 & 0.46 & 0.505 & 0.02 & 0.14 & 0.716 & 0.01 & 0.10 & 0.756 & 0.00 & 0.02 & 0.901 & 0.00 & 0.08 & 0.786 & 0.00 & 2.68 & 0.113 & 0.09 \\
\hline Heart Pounding & 3.03 & 0.093 & 0.10 & 0.03 & 0.863 & 0.00 & 0.82 & 0.373 & 0.03 & 0.32 & 0.576 & 0.01 & 0.06 & 0.808 & 0.00 & 0.05 & 0.832 & 0.00 & 2.08 & 0.160 & 0.07 \\
\hline Confused & 0.01 & 0.929 & 0.00 & 2.65 & 0.115 & 0.09 & 0.05 & 0.831 & 0.00 & 1.79 & 0.190 & 0.06 & 1.30 & 0.263 & 0.04 & 3.39 & 0.076 & 0.12 & 0.44 & 0.514 & 0.02 \\
\hline Weak & 2.68 & 0.113 & 0.09 & 2.58 & 0.119 & 0.08 & 3.79 & 0.062 & 0.12 & 0.26 & 0.617 & 0.01 & 0.26 & 0.617 & 0.01 & 0.20 & 0.656 & 0.01 & 1.85 & 0.184 & 0.06 \\
\hline \multicolumn{22}{|l|}{ Direct Effects of Product Use $\mathrm{e}^{\mathrm{b}}$} \\
\hline Satisfying & 0.67 & 0.419 & 0.02 & 0.09 & 0.765 & 0.00 & 1.14 & 0.294 & 0.04 & 0.74 & 0.397 & 0.03 & 0.00 & 0.962 & 0.00 & 1.86 & 0.184 & 0.06 & 3.88 & 0.059 & 0.12 \\
\hline Pleasant & 1.41 & 0.245 & 0.05 & 0.08 & 0.785 & 0.00 & 1.77 & 0.195 & 0.06 & 0.07 & 0.789 & 0.00 & 0.01 & 0.920 & 0.00 & 3.07 & 0.090 & 0.10 & 6.12 & 0.020 & 0.18 \\
\hline Taste Good & 1.93 & 0.180 & 0.06 & 0.16 & 0.696 & 0.01 & 0.74 & 0.396 & 0.03 & 0.62 & 0.437 & 0.02 & 1.39 & 0.248 & 0.05 & 1.02 & 0.320 & 0.04 & 0.03 & 0.860 & 0.00 \\
\hline Dizzy & 0.14 & 0.708 & 0.01 & 0.17 & 0.686 & 0.01 & 0.92 & 0.345 & 0.03 & 0.09 & 0.771 & 0.00 & 2.07 & 0.161 & 0.07 & 4.84 & 0.036 & 0.15 & 0.36 & 0.555 & 0.01 \\
\hline Calm & 0.73 & 0.400 & 0.03 & 0.66 & 0.420 & 0.02 & 2.23 & 0.147 & 0.07 & 0.20 & 0.658 & 0.01 & 0.17 & 0.682 & 0.01 & 0.01 & 0.911 & 0.00 & 0.37 & 0.546 & 0.01 \\
\hline Concentrate & 0.94 & 0.340 & 0.03 & 0.73 & 0.401 & 0.73 & 0.79 & 0.382 & 0.03 & 0.17 & 0.684 & 0.01 & 0.26 & 0.617 & 0.01 & 0.23 & 0.636 & 0.01 & 1.52 & 0.228 & 0.05 \\
\hline Awake & 0.00 & 0.990 & 0.00 & 1.94 & 0.175 & 0.07 & 0.14 & 0.710 & 0.01 & 0.07 & 0.797 & 0.00 & 0.00 & 0.948 & 0.00 & 0.48 & 0.496 & 0.02 & 0.01 & 0.921 & 0.00 \\
\hline Reduced Hunger & 0.68 & 0.420 & 0.02 & 2.08 & 0.160 & 0.07 & 0.71 & 0.406 & 0.03 & 0.42 & 0.520 & 0.02 & 0.22 & 0.645 & 0.01 & 0.04 & 0.845 & 0.00 & 0.04 & 0.843 & 0.00 \\
\hline Sick & 0.03 & 0.864 & 0.00 & 7.47 & 0.011 & 0.21 & 1.49 & 0.232 & 0.05 & 0.16 & 0.695 & 0.01 & 0.00 & 0.953 & 0.00 & 0.32 & 0.574 & 0.01 & 0.65 & 0.428 & 0.02 \\
\hline \multicolumn{22}{|l|}{ gLMS $^{\mathrm{c}}$} \\
\hline Sensation & 0.53 & 0.480 & 0.03 & 4.73 & 0.046 & 0.24 & & & & 0.83 & 0.377 & 0.05 & & & & & & & & & \\
\hline Harshness & 0.00 & 0.980 & 0.00 & 0.00 & 0.988 & 0.00 & & & & 0.33 & 0.577 & 0.02 & & & & & & & & & \\
\hline Throat Hit & 0.68 & 0.424 & 0.04 & 1.70 & 0.212 & 0.10 & & & & 0.51 & 0.484 & 0.03 & & & & & & & & & \\
\hline Puff Duration ${ }^{\mathrm{b}}$ & 0.84 & 0.366 & 0.03 & 0.46 & 0.505 & 0.02 & & & & 0.17 & 0.685 & 0.01 & & & & & & & & & \\
\hline
\end{tabular}

Table 4 
Repeated Measure ANOVA Results by Order

\begin{tabular}{|c|c|c|c|c|c|c|c|c|c|c|c|c|c|c|c|c|c|c|c|c|c|}
\hline \multirow[b]{2}{*}{ Outcome Measure } & \multicolumn{3}{|c|}{ Order } & \multicolumn{3}{|c|}{ Bout } & \multicolumn{3}{|c|}{ Time } & \multicolumn{3}{|c|}{ Order $x$ Bout } & \multicolumn{3}{|c|}{ Order $\mathrm{x}$ Time } & \multicolumn{3}{|c|}{ Bout $\mathrm{x}$ Time } & \multicolumn{3}{|c|}{ Order $\mathrm{x}$ Bout $\mathrm{x}$ Time } \\
\hline & $F$ & $p$ & $\eta_{p}^{2}$ & $F$ & $p$ & $\eta_{p}^{2}$ & $F$ & $p$ & $\eta_{p}^{2}$ & $F$ & $p$ & $\eta_{\mathrm{p}}{ }^{2}$ & $F$ & $p$ & $\eta_{p}^{2}$ & $F$ & $p$ & $\eta_{p}^{2}$ & $F$ & $p$ & $\eta_{p}^{2}$ \\
\hline \multicolumn{22}{|l|}{ Physiological Measures $^{\mathrm{a}}$} \\
\hline Heart Rate & 0.00 & 0.960 & 0.00 & 21.98 & 0.000 & 0.45 & 15.77 & 0.000 & 0.37 & 0.26 & 0.620 & 0.01 & 0.07 & 0.800 & 0.00 & 2.92 & 0.100 & 0.10 & 12.61 & 0.001 & 0.32 \\
\hline Systolic & 1.84 & 0.190 & 0.06 & 0.34 & 0.570 & 0.01 & 10.47 & 0.003 & 0.28 & 0.01 & 0.920 & 0.00 & 0.69 & 0.420 & 0.03 & 0.05 & 0.830 & 0.00 & 1.22 & 0.280 & 0.04 \\
\hline Diastolic & 1.23 & 0.280 & 0.04 & 0.55 & 0.470 & 0.02 & 6.41 & 0.020 & 0.19 & 0.60 & 0.450 & 0.02 & 0.04 & 0.840 & 0.00 & 0.07 & 0.800 & 0.00 & 2.96 & 0.100 & 0.10 \\
\hline \multicolumn{22}{|l|}{ Direct Effects of Nicotine ${ }^{\mathrm{b}}$} \\
\hline Dizzy & 7.47 & 0.010 & 0.21 & 2.03 & 0.170 & 0.07 & 19.99 & 0.000 & 0.41 & 0.34 & 0.570 & 0.01 & 0.27 & 0.610 & 0.01 & 3.49 & 0.070 & 0.12 & 3.17 & 0.090 & 0.10 \\
\hline Lightheaded & 12.44 & 0.001 & 0.30 & 5.61 & 0.030 & 0.16 & 33.09 & 0.000 & 0.53 & 0.01 & 0.910 & 0.00 & 0.48 & 0.490 & 0.02 & 2.01 & 0.170 & 0.07 & 3.73 & 0.060 & 0.11 \\
\hline Nauseaus & 7.18 & 0.010 & 0.20 & 0.03 & 0.860 & 0.00 & 14.18 & 0.001 & 0.33 & 0.00 & 0.950 & 0.00 & 0.51 & 0.480 & 0.02 & 0.05 & 0.820 & 0.00 & 1.65 & 0.210 & 0.05 \\
\hline Nervous & 0.83 & 0.370 & 0.03 & 6.96 & 0.010 & 0.19 & 0.59 & 0.450 & 0.02 & 0.04 & 0.840 & 0.00 & 0.30 & 0.590 & 0.01 & 2.69 & 0.110 & 0.09 & 0.74 & 0.400 & 0.03 \\
\hline Sweaty & 0.07 & 0.800 & 0.00 & 2.04 & 0.160 & 0.07 & 0.43 & 0.520 & 0.02 & 0.33 & 0.570 & 0.01 & 0.22 & 0.650 & 0.01 & 5.92 & 0.020 & 0.17 & 0.35 & 0.560 & 0.01 \\
\hline Headache & 0.87 & 0.360 & 0.03 & 3.20 & 0.080 & 0.10 & 3.06 & 0.090 & 0.10 & 0.08 & 0.790 & 0.00 & 0.28 & 0.600 & 0.01 & 0.06 & 0.810 & 0.00 & 0.19 & 0.670 & 0.01 \\
\hline Excess Salivation & 2.88 & 0.100 & 0.09 & 1.09 & 0.300 & 0.04 & 2.19 & 0.150 & 0.07 & 0.60 & 0.450 & 0.02 & 3.86 & 0.060 & 0.12 & 0.32 & 0.580 & 0.01 & 0.29 & 0.590 & 0.01 \\
\hline Heart Pounding & 0.11 & 0.750 & 0.00 & 4.35 & 0.046 & 0.13 & 2.67 & 0.110 & 0.08 & 0.15 & 0.710 & 0.01 & 0.02 & 0.900 & 0.00 & 1.98 & 0.170 & 0.06 & 0.04 & 0.840 & 0.00 \\
\hline Confused & 3.29 & 0.080 & 0.10 & 0.05 & 0.810 & 0.00 & 2.19 & 0.150 & 0.07 & 0.63 & 0.430 & 0.02 & 0.68 & 0.420 & 0.02 & 0.25 & 0.620 & 0.01 & 0.16 & 0.690 & 0.01 \\
\hline Weak & 4.70 & 0.040 & 0.14 & 0.10 & 0.760 & 0.00 & 4.51 & 0.040 & 0.14 & 1.77 & 0.190 & 0.06 & 1.00 & 0.320 & 0.03 & 2.23 & 0.150 & 0.07 & 3.13 & 0.090 & 0.10 \\
\hline \multicolumn{22}{|l|}{ Direct Effects of Product Use ${ }^{b}$} \\
\hline Satisfying & 0.10 & 0.760 & 0.00 & 18.08 & 0.000 & 0.38 & 44.97 & 0.000 & 0.61 & 0.00 & 0.950 & 0.00 & 0.44 & 0.520 & 0.02 & 15.62 & 0.000 & 0.35 & 0.00 & 0.950 & 0.00 \\
\hline Pleasant & 0.77 & 0.390 & 0.03 & 23.08 & 0.000 & 0.44 & 36.36 & 0.000 & 0.56 & 0.51 & 0.480 & 0.02 & 0.05 & 0.830 & 0.00 & 12.73 & 0.001 & 0.31 & 0.35 & 0.560 & 0.01 \\
\hline Taste Good & 3.81 & 0.060 & 0.12 & 18.89 & 0.000 & 0.39 & 41.40 & 0.000 & 0.59 & 0.11 & 0.740 & 0.00 & 3.72 & 0.060 & 0.11 & 23.96 & 0.000 & 0.45 & 0.72 & 0.400 & 0.02 \\
\hline Dizzy & 3.72 & 0.060 & 0.11 & 20.81 & 0.000 & 0.42 & 37.50 & 0.000 & 0.56 & 2.03 & 0.170 & 0.07 & 0.07 & 0.800 & 0.00 & 13.67 & 0.001 & 0.32 & 2.59 & 0.120 & 0.08 \\
\hline Calm & 9.60 & 0.004 & 0.25 & 11.38 & 0.002 & 0.28 & 23.50 & 0.000 & 0.45 & 1.17 & 0.290 & 0.04 & 7.41 & 0.010 & 0.20 & 17.17 & 0.000 & 0.37 & 1.04 & 0.320 & 0.03 \\
\hline Concentrate & 0.12 & 0.730 & 0.00 & 9.38 & 0.010 & 0.24 & 15.32 & 0.001 & 0.35 & 0.01 & 0.920 & 0.00 & 0.01 & 0.940 & 0.00 & 3.25 & 0.080 & 0.10 & 0.04 & 0.840 & 0.00 \\
\hline Awake & 1.17 & 0.290 & 0.04 & 8.60 & 0.010 & 0.23 & 26.15 & 0.000 & 0.47 & 0.83 & 0.370 & 0.03 & 3.20 & 0.080 & 0.10 & 8.56 & 0.010 & 0.23 & 0.10 & 0.750 & 0.00 \\
\hline Reduced Hunger & 5.40 & 0.030 & 0.16 & 9.20 & 0.010 & 0.24 & 35.74 & 0.000 & 0.55 & 0.06 & 0.810 & 0.00 & 2.83 & 0.100 & 0.09 & 16.03 & 0.000 & 0.36 & 2.12 & 0.160 & 0.07 \\
\hline Sick & 5.49 & 0.030 & 0.16 & 5.74 & 0.020 & 0.17 & 13.00 & 0.001 & 0.31 & 0.77 & 0.390 & 0.03 & 11.37 & 0.002 & 0.28 & 13.99 & 0.001 & 0.33 & 3.34 & 0.080 & 0.10 \\
\hline \multicolumn{22}{|l|}{ gLMS $^{\mathrm{c}}$} \\
\hline Sensation & 0.00 & 0.970 & 0.00 & 1.86 & 0.190 & 0.10 & & & & 0.77 & 0.390 & 0.05 & & & & & & & & & \\
\hline Harshness & 0.07 & 0.790 & 0.01 & 2.75 & 0.120 & 0.15 & & & & 0.50 & 0.490 & 0.03 & & & & & & & & & \\
\hline Throat Hit & 0.00 & 1.000 & 0.00 & 0.91 & 0.350 & 0.05 & & & & 0.01 & 0.920 & 0.00 & & & & & & & & & \\
\hline Puff Duration ${ }^{\mathrm{b}}$ & 0.04 & 0.850 & 0.00 & 11.27 & 0.002 & 0.28 & & & & 3.92 & 0.060 & 0.12 & & & & & & & & & \\
\hline
\end{tabular}

Table 5 


\section{Mixed Repeated measure ANOVAs for Behavioral Economics Outcomes}

\begin{tabular}{|c|c|c|c|c|c|c|}
\hline \multirow[b]{2}{*}{ Outcome Measure } & \multicolumn{3}{|c|}{ Flavor } & \multicolumn{3}{|c|}{ Flavor x Race } \\
\hline & $F$ & $p$ & $\eta_{\mathrm{p}}^{2}$ & $F$ & $p$ & $\eta_{p}^{2}$ \\
\hline \multicolumn{7}{|l|}{ PRT $^{\mathrm{a}}$} \\
\hline Breakpoint & 0.14 & 0.712 & 0.01 & 0.38 & 0.542 & 0.01 \\
\hline Rewards earned & 1.49 & 0.233 & 0.05 & 3.84 & 0.060 & 0.12 \\
\hline Total responses & 0.04 & 0.852 & 0.00 & 0.19 & 0.665 & 0.01 \\
\hline Responses per minute & 0.09 & 0.766 & 0.00 & 0.29 & 0.594 & 0.01 \\
\hline Latency to puff ${ }^{b}$ & 0.19 & 0.670 & 0.02 & 0.58 & 0.462 & 0.05 \\
\hline \multicolumn{7}{|l|}{$\mathrm{PBPT}^{\mathrm{a}}$} \\
\hline Breakpoint $^{\mathrm{c}}$ & 0.01 & 0.928 & 0.00 & 0.02 & 0.889 & 0.00 \\
\hline Intensity & 0.27 & 0.609 & 0.01 & 0.00 & 0.990 & 0.00 \\
\hline AUC & 0.01 & 0.918 & 0.00 & 0.04 & 0.850 & 0.00 \\
\hline Elasticity & 0.13 & 0.720 & 0.01 & 0.10 & 0.757 & 0.00 \\
\hline
\end{tabular}

${ }^{a} d f=1,28 ;{ }^{b} d f=1,11 ;{ }^{c} d f=1,26$

Table 6 
Means and Standard Deviations for Behavioral Economics Outcomes

\begin{tabular}{lcc}
\hline & Fruit & Tobacco \\
\hline Rewards Earned & $3.48(5.67)$ & $3.52(6.08)$ \\
Breakpoint & $112.83(248.22)$ & $136.20(298.42)$ \\
Total responses & $395.6(1004.92)$ & $439.57(1063.98)$ \\
Responses per minute & $19.78(50.25)$ & $22.93(53.13)$ \\
Latency to puff & $3.98(1.00)$ & $3.75(1.00)$ \\
\hline Breakpoint & $68.93(77.98)$ & $75.11(83.98)$ \\
Demand Intensity & $62.83(41.48)$ & $66.67(36.14)$ \\
Demand elasticity & $0.0004(.002)$ & $.001(.007)$ \\
AUC & $0.06(.08)$ & $.06(.06)$ \\
\hline
\end{tabular}

Table 7 
Means and Standard Deviations for ECIG Outcome Expectancies

\begin{tabular}{rc}
\hline & $M(S D)$ \\
\hline Positive Score & $11.83(7.04)$ \\
\hline Become more popular & $2.33(2.45)$ \\
Feel relaxed & $3.03(2.53)$ \\
Enjoy "smoking" without bothering others & $3.73(2.75)$ \\
Smell Good & $2.73(2.46)$ \\
\hline Negative Score & $21.7(4.35)$ \\
\hline Hurt Lungs & $6.97(2.08)$ \\
Look Awkward & $3.37(2.59)$ \\
Become Addicted & $6.67(2.37)$ \\
Bad Taste & $4.7(2.34)$ \\
\hline
\end{tabular}


Table 8

Frequencies Flavor Preferences from Participant Exit Interviews

\begin{tabular}{rcc}
\hline Flavors & Prefer & Dislike \\
\hline Mint/menthol & 10 & 9 \\
Tobacco & 0 & 8 \\
Mango & 7 & 0 \\
Berry/berry medley & 5 & 3 \\
Crème brulee & 2 & 1 \\
Cereal/Drink & 4 & 1 \\
Other & 4 & 5 \\
None & 6 & 10 \\
N/A (did not remember) & 2 & 1 \\
\hline
\end{tabular}


Table 9

Themes and Response Summaries from Participant Exit Interviews

\begin{tabular}{|c|c|c|c|}
\hline Reasons for liking flavors & Reasons for disliking flavors & Settings for product use & Reasons for product use \\
\hline \multirow[t]{3}{*}{$\begin{array}{l}\text { Prefers cucumber because it } \\
\text { tasted very clean. Also liked } \\
\text { crème brulee and mango } \\
\text { because those two did not hurt } \\
\text { and were not accompanied by } \\
\text { burn from the ECIG. }\end{array}$} & $\begin{array}{l}\text { Virginia tobacco and tobacco are } \\
\text { sickness-inducing and lead to } \\
\text { feeling lightheaded, clammy, and } \\
\text { like they are about to pass out. } \\
\text { Can make their stomach feel upset. }\end{array}$ & $\begin{array}{l}\text { At a party, at home, in the car driving } \\
\text { around, a concert, usually with } \\
\text { people. Doesn't enjoy doing it by } \\
\text { themselves. Feel more social than it } \\
\text { is relaxing. }\end{array}$ & $\begin{array}{c}\text { Just to taste different flavors. Usually } \\
\text { someone asks if I want to try a different } \\
\text { flavor, I never seek it out. }\end{array}$ \\
\hline & $\begin{array}{l}\text { Disliked mint flavor because it } \\
\text { burned. }\end{array}$ & $\begin{array}{l}\text { Only at parties when alcohol is } \\
\text { involved }\end{array}$ & $\begin{array}{l}\text { First few times they liked getting a buzz and } \\
\text { wanted to try to make smoke rings. With } \\
\text { ECIGS they mostly enjoy the buzz and the } \\
\text { taste. When other people are using an } \\
\text { ECIG, it makes them want to do it. }\end{array}$ \\
\hline & $\begin{array}{l}\text { Didn't like crème brulee because } \\
\text { they don't like caramel. Hot tamale } \\
\text { was too strong. Mint tasted like a } \\
\text { throat hit. Too harsh. }\end{array}$ & Drunk, usually at a bar or at the lake. & $\begin{array}{l}\text { Pressure from roommates who said it } \\
\text { wasn't that bad. They decided to try it } \\
\text { again in different flavors, because they were } \\
\text { told that they did it wrong the first time. }\end{array}$ \\
\hline
\end{tabular}




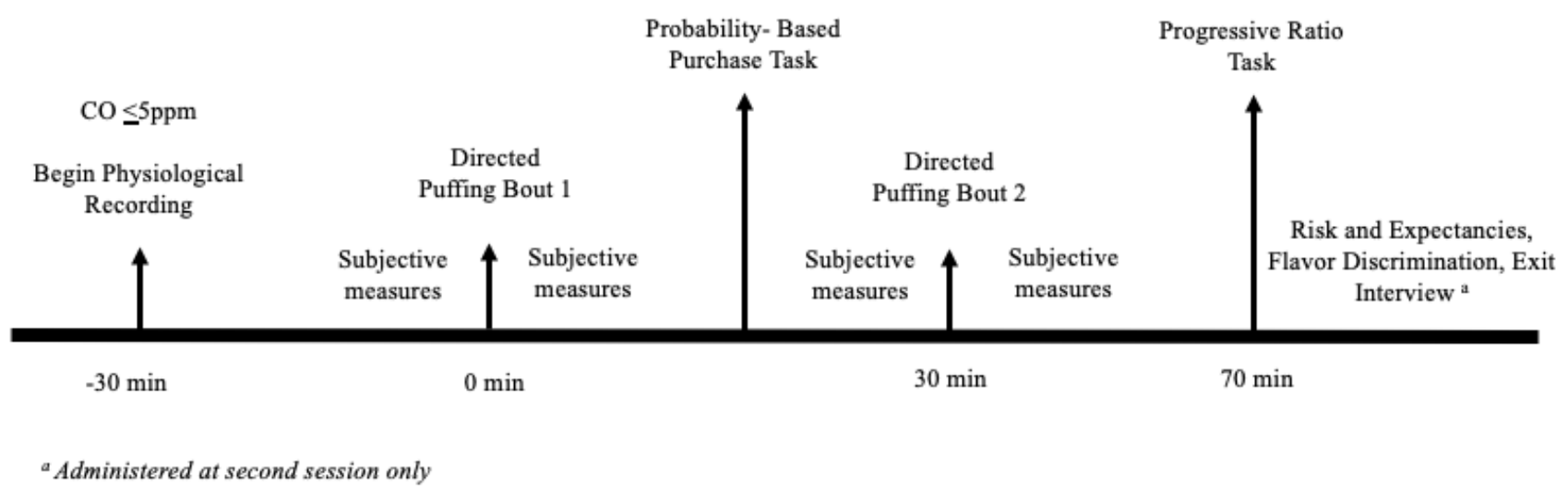

Figure 1. Session procedure. 


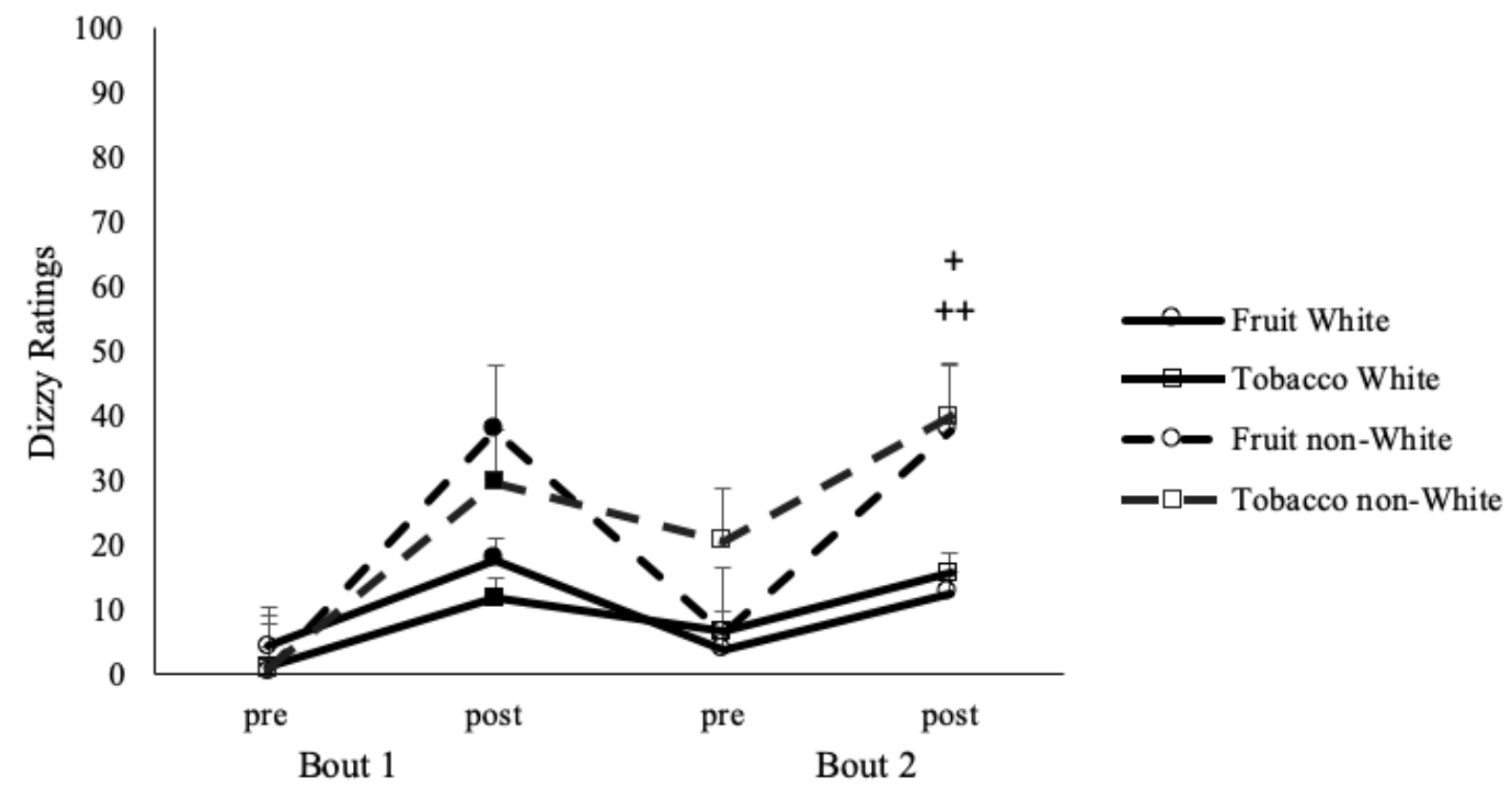

Figure 2. Mean (+/- SEMs) ratings are shown for the DEPS item "dizzy" for bout $\mathrm{x}$ time $\mathrm{x}$ race effects as a function of flavor (fruit vs. tobacco) x bout (1 vs. 2) x time (pre vs. post) for White and non-White participants. Filled symbols = significant differences pre- to post- within bout; $+=$ significant differences between race groups for fruit flavor within timepoint; $++=$ significant differences between race groups for tobacco flavor within timepoint. 


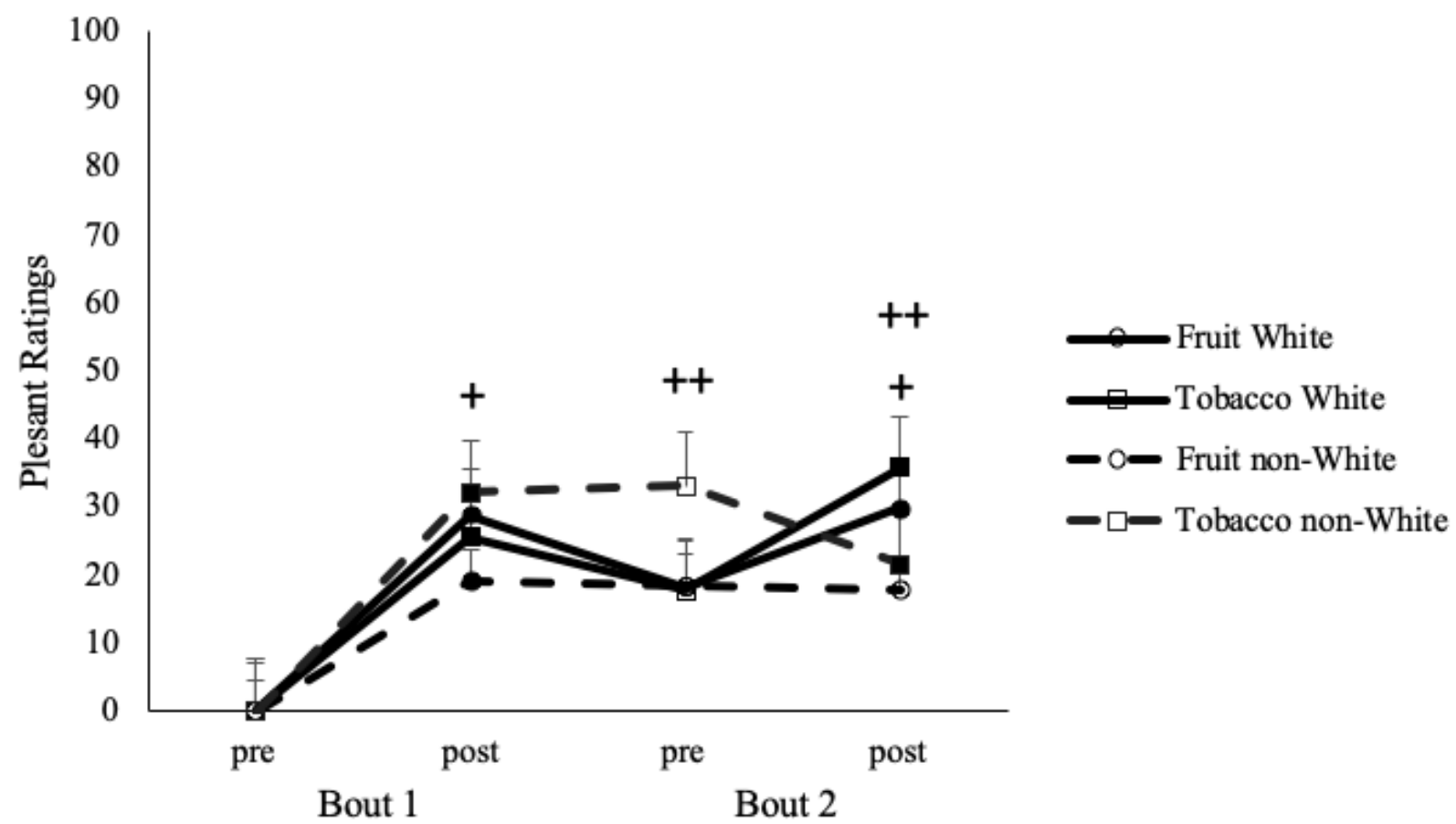

Figure 3. Mean (+/- SEMs) ratings are shown for the DEPS item "pleasant" for flavor $\mathrm{x}$ bout $\mathrm{x}$ time $x$ race effects as a function of flavor (fruit vs. tobacco) x bout (1 vs. 2) x time (pre vs. post) for White and non-White participants. Filled symbols = significant differences pre- to post- within bout; $+=$ significant differences between race groups for fruit flavor within timepoint; $++=$ significant differences between race groups for tobacco flavor within timepoint. 


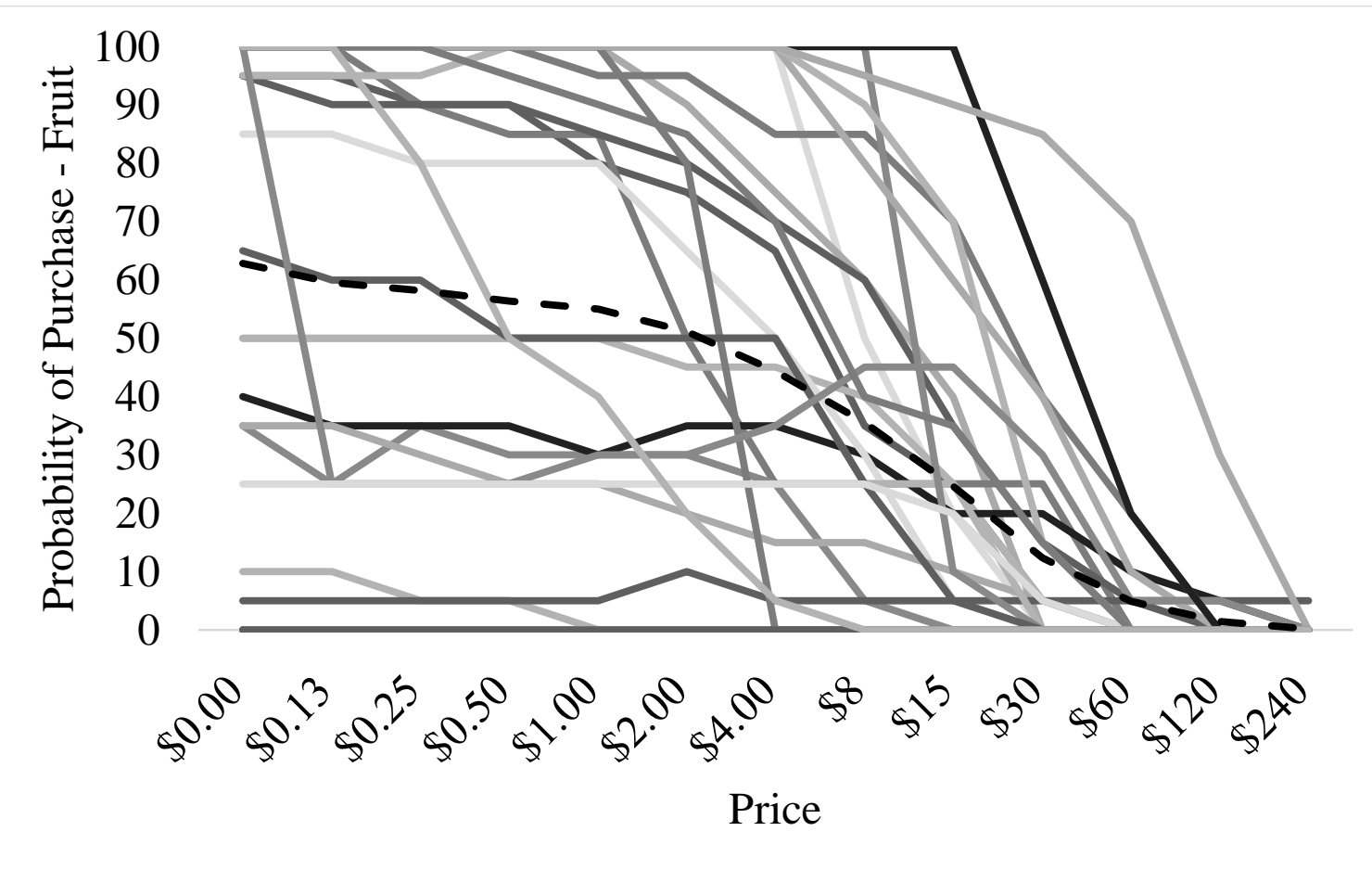

Figure 4. Fruit flavor demand curve for probability-based purchase task. Probability of purchase across a range of prices is depicted for all participants in the fruit flavor condition. Dashed line represents average demand curve. 


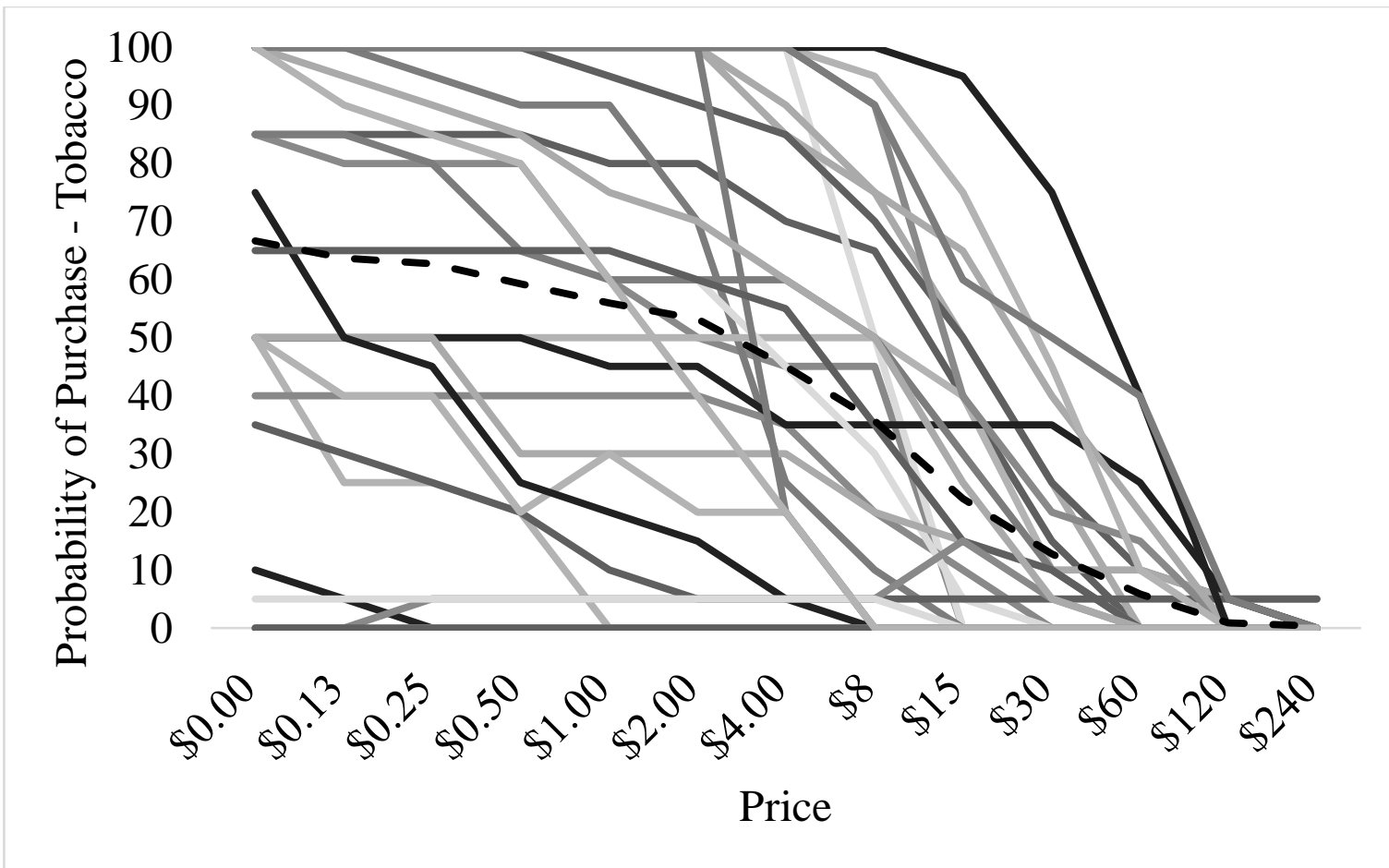

Figure 5. Tobacco flavor demand curve for probability-based purchase task. Probability of purchase across a range of prices is depicted for all participants in the tobacco flavor condition. Dashed line represents average demand curve. 


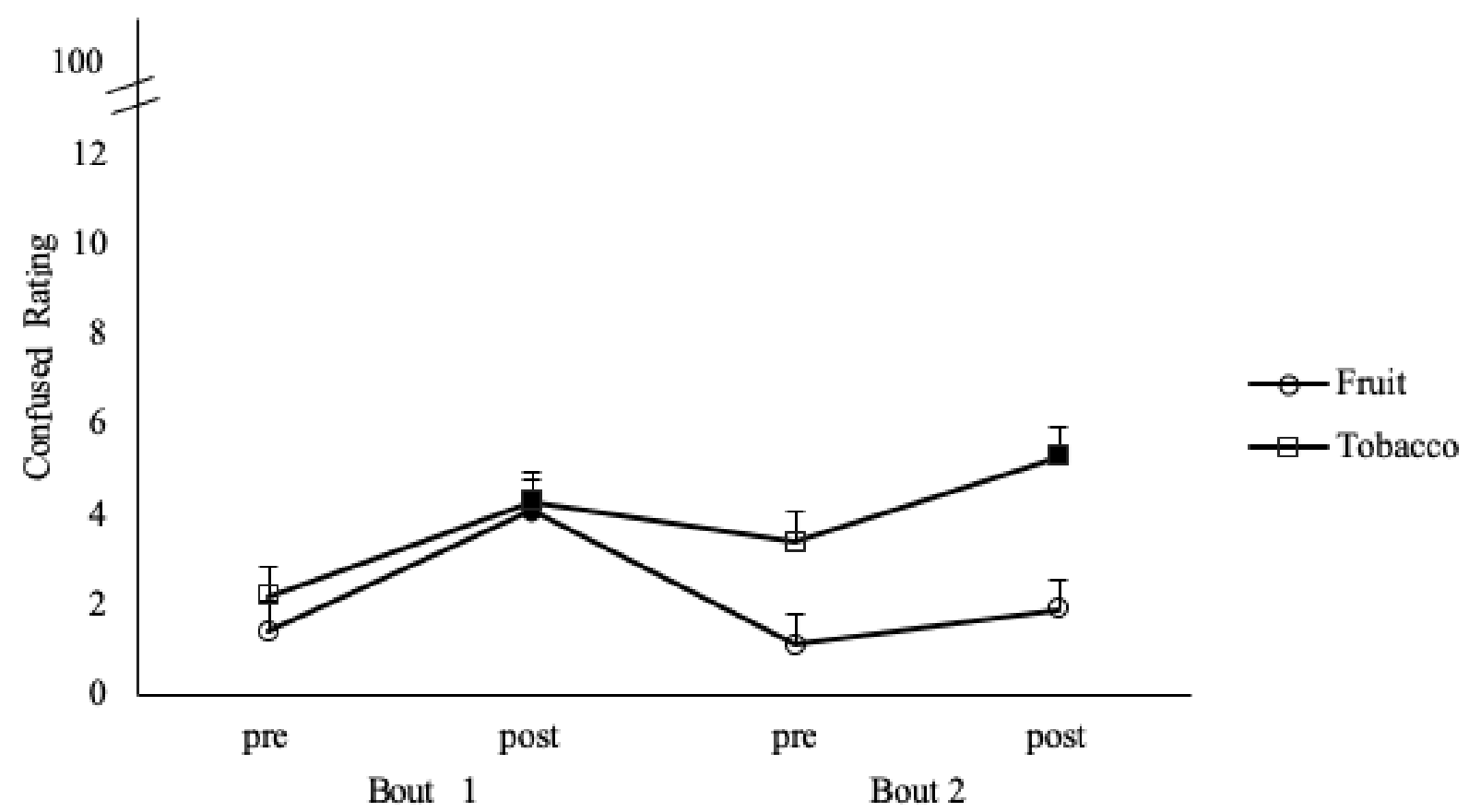

Figure 6. Mean (+/- SEMs) ratings are shown for the DENS item "confused" for flavor $\mathrm{x}$ bout $\mathrm{x}$ time effects as a function of flavor (fruit vs. tobacco) x bout (1 vs. 2$) \times$ time (pre vs. post). Filled symbols $=$ significant differences pre- to post- within bout. 


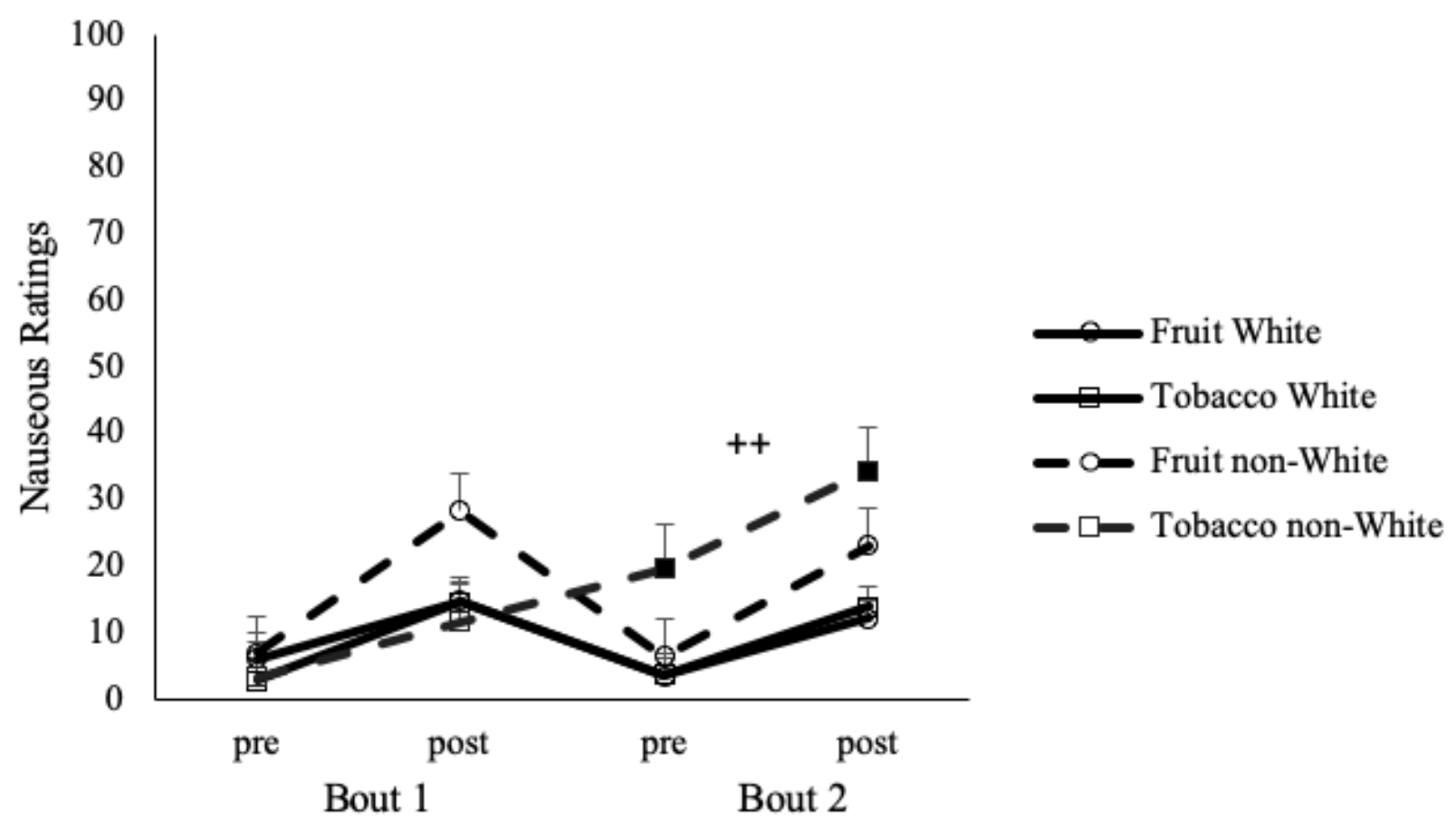

Figure 7. Mean (+/- SEMs) ratings are shown for the DENS item "nauseous" for flavor $\mathrm{x}$ bout $\mathrm{x}$ race effects as a function of flavor (fruit vs. tobacco) x bout (1 vs. 2) x time (pre vs. post) for White and non-White participants. Filled symbols = significant differences between bouts; $+=$ significant differences between race groups for fruit flavor within bout; $++=$ significant differences between race groups for tobacco flavor within bout. 


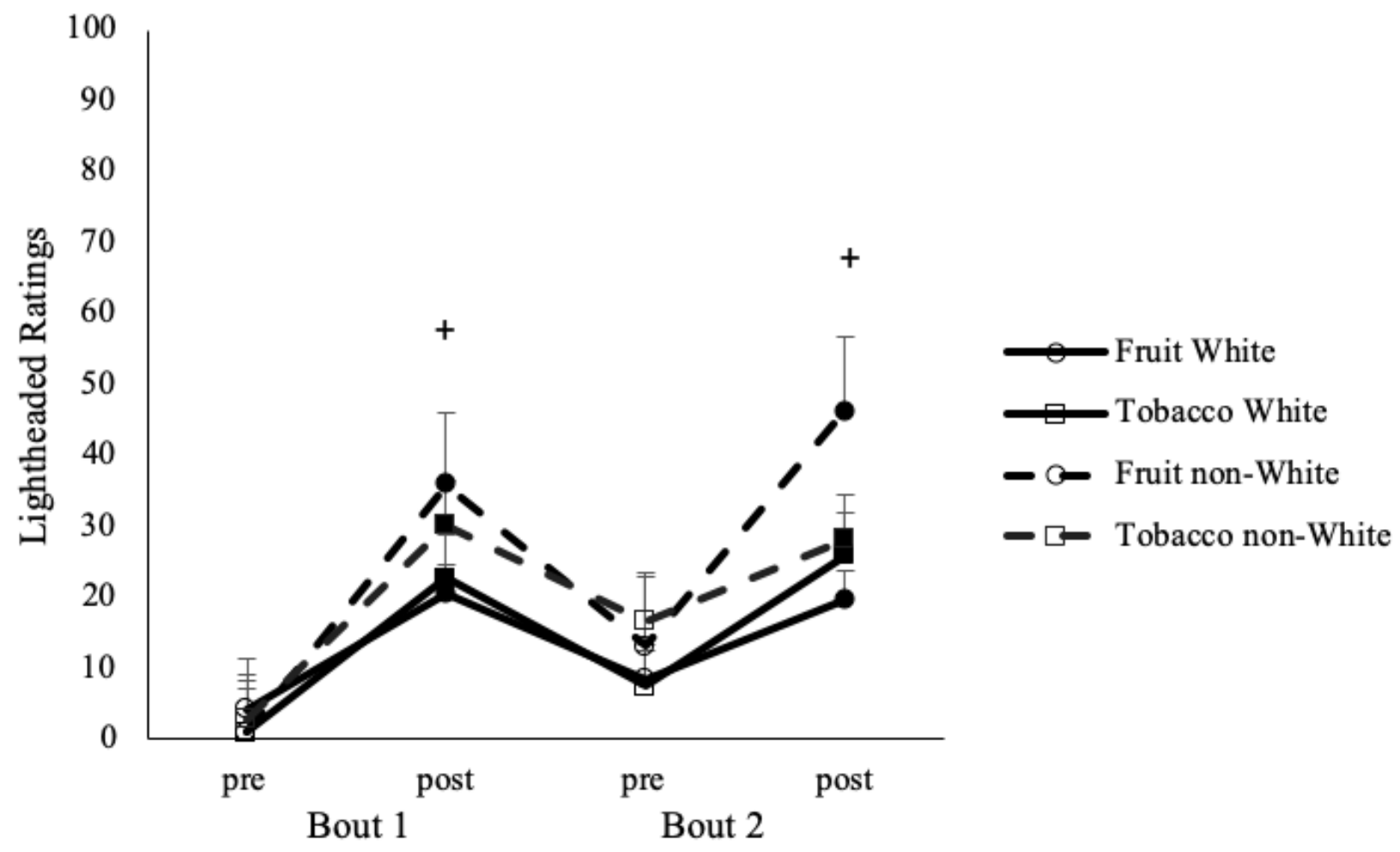

Figure 8. Mean (+/- SEMs) ratings are shown for the DENS item "lightheaded" for flavor $\mathrm{x}$ time $\mathrm{x}$ race effects as a function of flavor (fruit vs. tobacco) $\mathrm{x}$ bout (1 vs. 2 ) $\mathrm{x}$ time (pre vs. post) for White and non-White participants. Filled symbols = significant differences pre- to post- within bout; $+=$ significant differences between race groups for fruit flavor within timepoint; $++=$ significant differences between race groups for tobacco flavor within timepoint. 


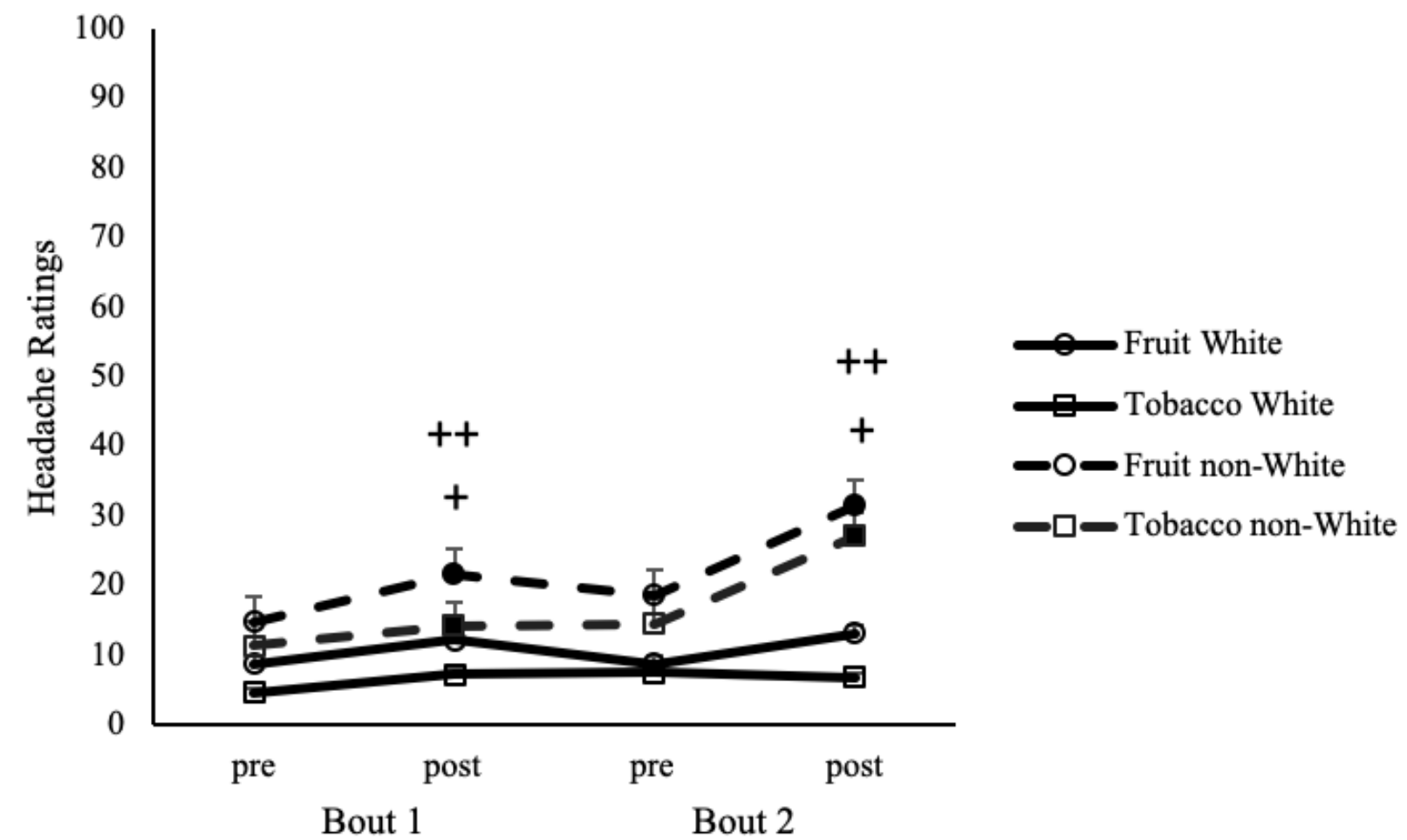

Figure 9. Mean (+/- SEMs) ratings are shown for the DENS item "headache" for bout $\mathrm{x}$ time $\mathrm{x}$ race effects as a function of flavor (fruit vs. tobacco) $\mathrm{x}$ bout (1 vs. 2) x time (pre vs. post) for White and non-White participants. Filled symbols = significant differences pre- to post- within bout; $+=$ significant differences between race groups for fruit flavor within timepoint; $++=$ significant differences between race groups for tobacco flavor within timepoint. 


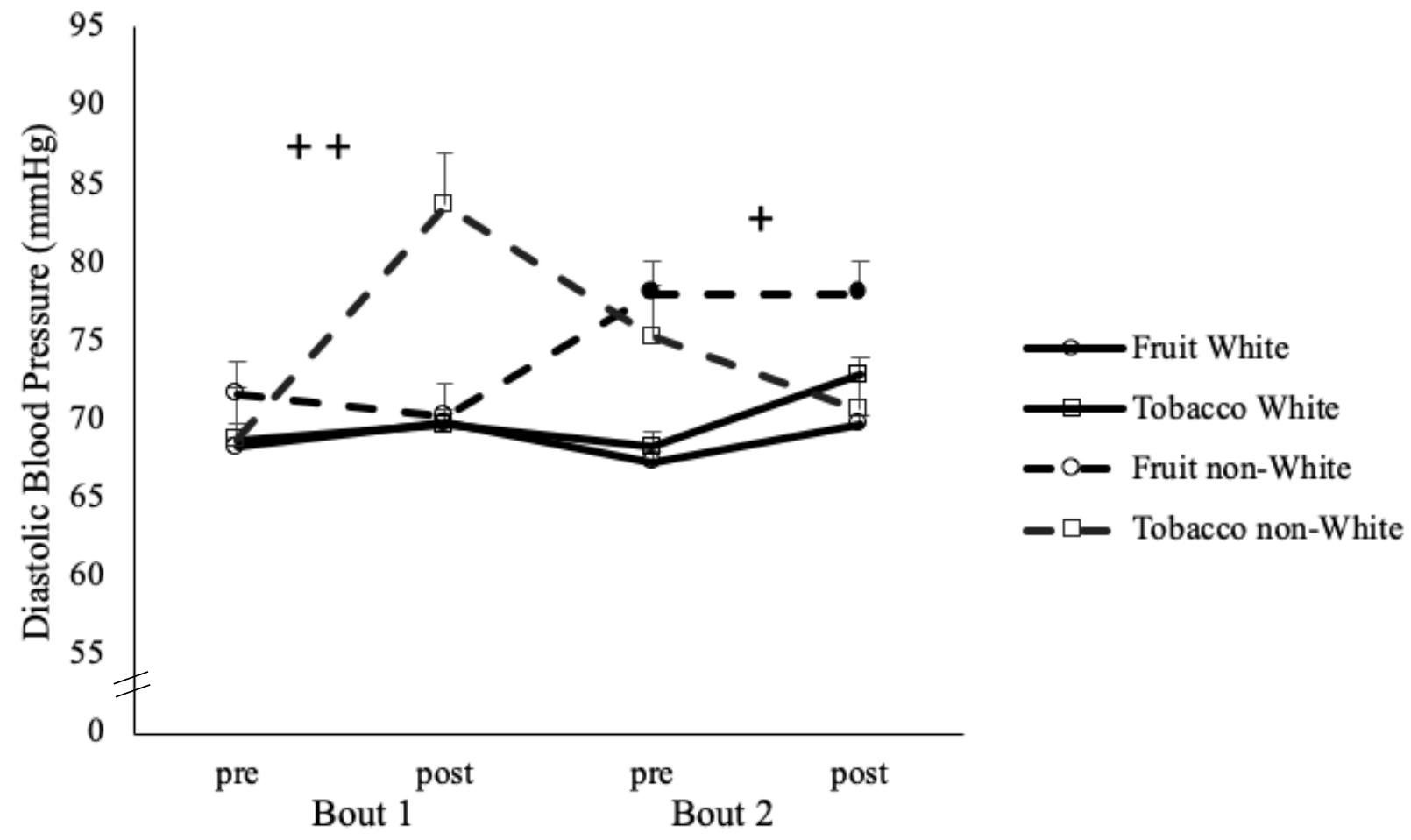

Figure 10. Mean (+/- SEMs) ratings are shown for diastolic blood pressure for flavor $\mathrm{x}$ bout $\mathrm{x}$ race effects as a function of flavor (fruit vs. tobacco) x bout (1 vs. 2) x time (pre vs. post) for White and non-White participants. Filled symbols = significant differences between bouts; + $=$ significant differences between race groups for fruit flavor within bout; $++=$ significant differences between race groups for tobacco flavor within bout. 


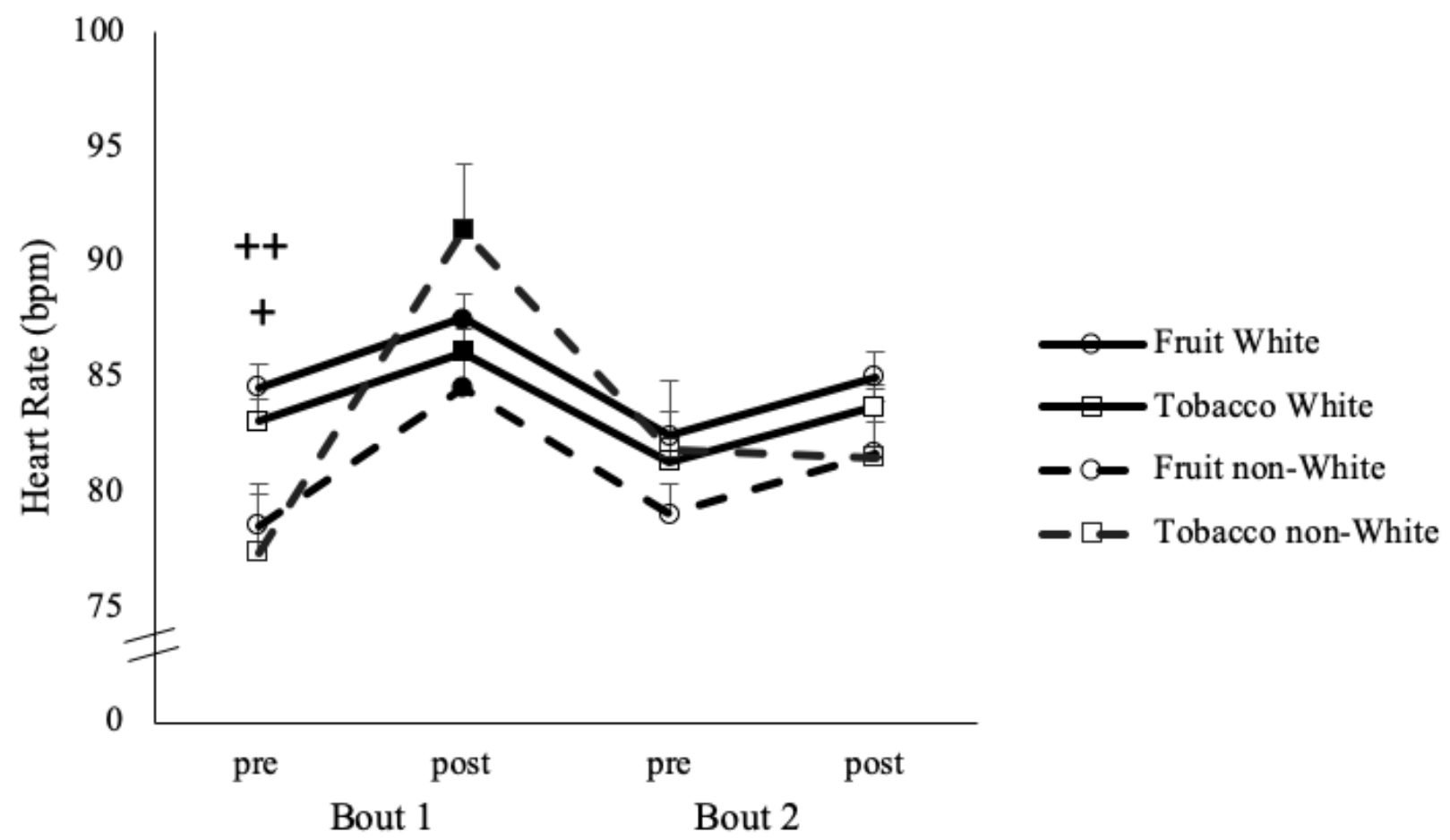

Figure 11. Mean (+/- SEMs) ratings are shown for heart rate for bout $\mathrm{x}$ time $\mathrm{x}$ race effects as a function of flavor (fruit vs. tobacco) x bout (1 vs. 2) x time (pre vs. post) for White and nonWhite participants. Filled symbols $=$ significant differences pre- to post- within bout; $+=$ significant differences between race groups for fruit flavor within timepoint; $++=$ significant differences between race groups for tobacco flavor within timepoint. 


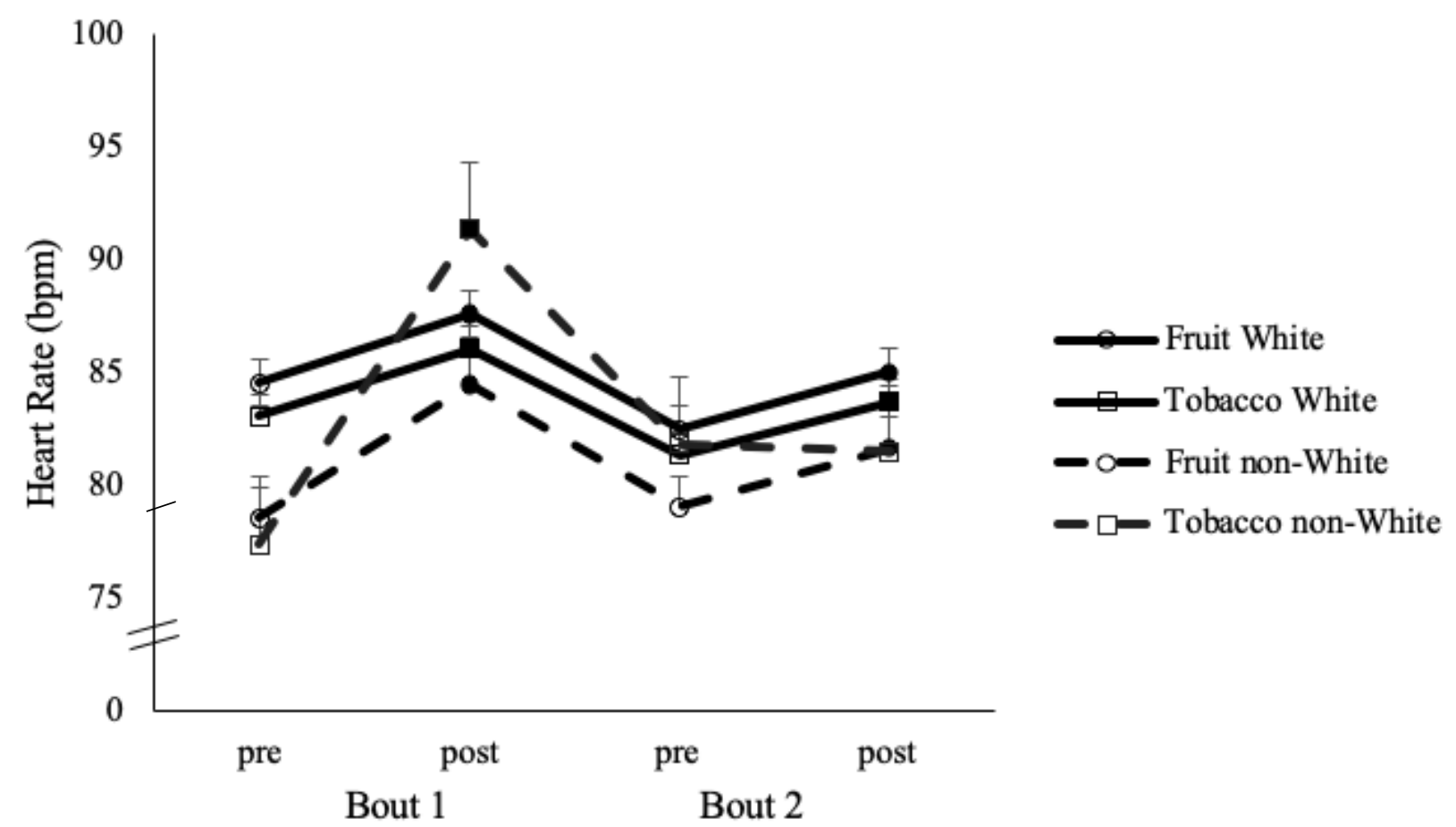

Figure 12. Mean (+/- SEMs) ratings are shown for heart rate for flavor $\mathrm{x}$ bout $\mathrm{x}$ time $\mathrm{x}$ race effects as a function of flavor (fruit vs. tobacco) x bout (1 vs. 2) x time (pre vs. post) for White and non-White participants. Filled symbols = significant differences pre- to postwithin bout; $+=$ significant differences between race groups for fruit flavor within timepoint; $++=$ significant differences between race groups for tobacco flavor within timepoint. 


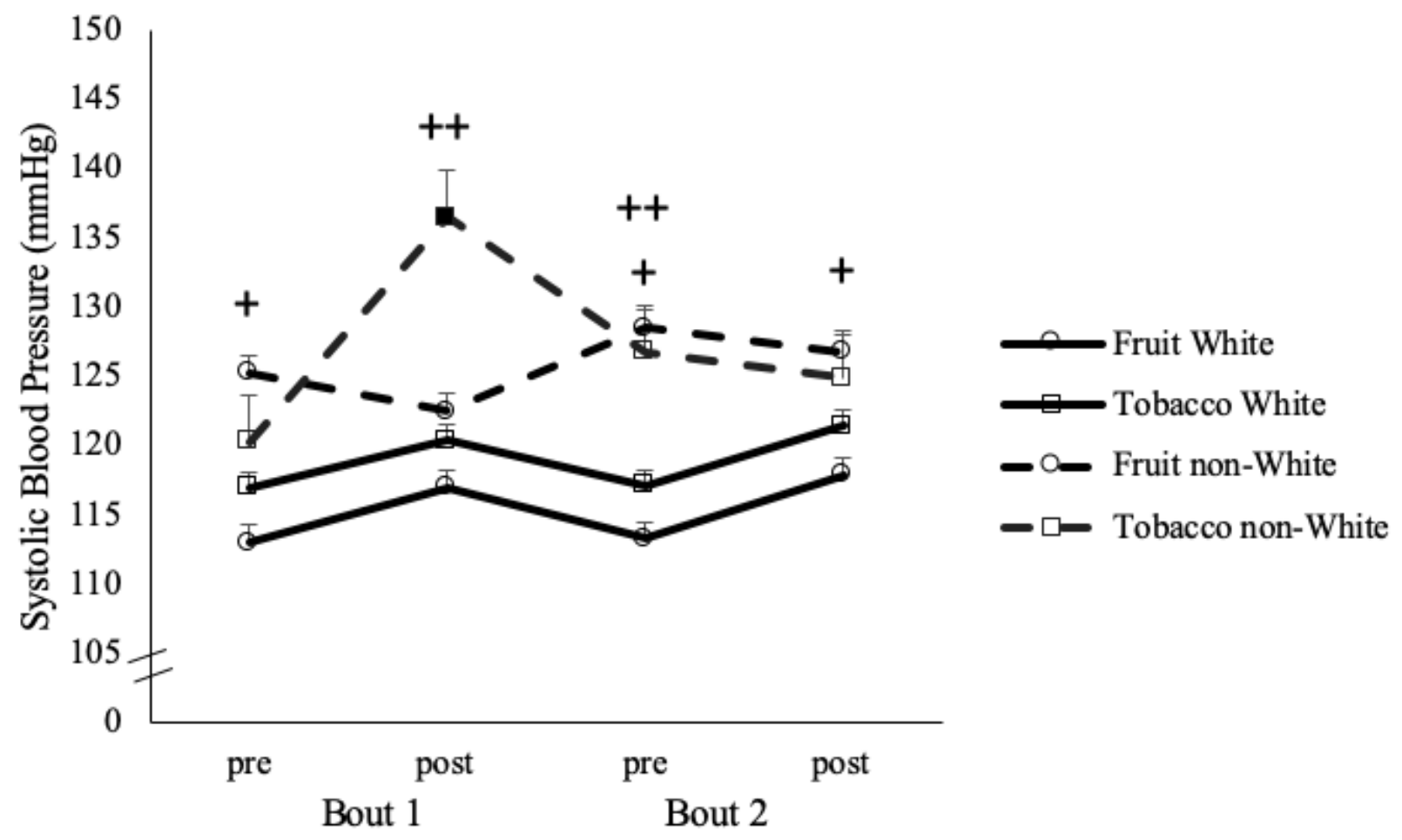

Figure 13. Mean (+/- SEMs) ratings are shown for systolic blood pressure for flavor $\mathrm{x}$ bout $\mathrm{x}$ time $\mathrm{x}$ race effects as a function of flavor (fruit vs. tobacco) x bout (1 vs. 2) x time (pre vs. post) for White and non-White participants. Filled symbols = significant differences pre- to post- within bout; $+=$ significant differences between race groups for fruit flavor within timepoint; $++=$ significant differences between race groups for tobacco flavor within timepoint. 


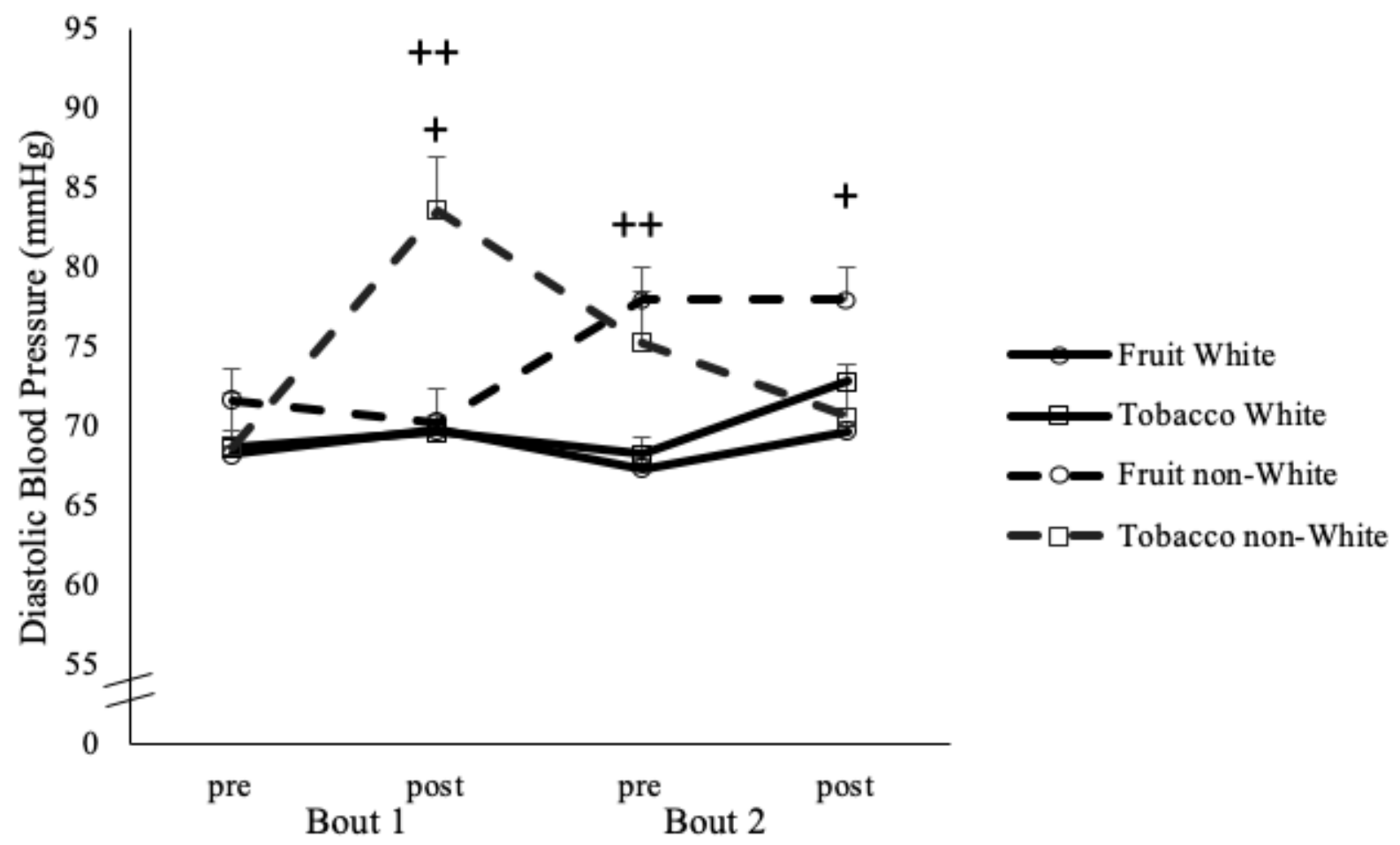

Figure 14. Mean (+/- SEMs) ratings are shown for diastolic blood pressure for flavor $\mathrm{x}$ bout $\mathrm{x}$ time $\mathrm{x}$ race as a function of flavor (fruit vs. tobacco) $\mathrm{x}$ bout (1 vs. 2) $\mathrm{x}$ time (pre vs. post) for White and non-White participants. Filled symbols = significant differences pre- to postwithin bout; $+=$ significant differences between race groups for fruit flavor within timepoint; $++=$ significant differences between race groups for tobacco flavor within timepoint or bout. 


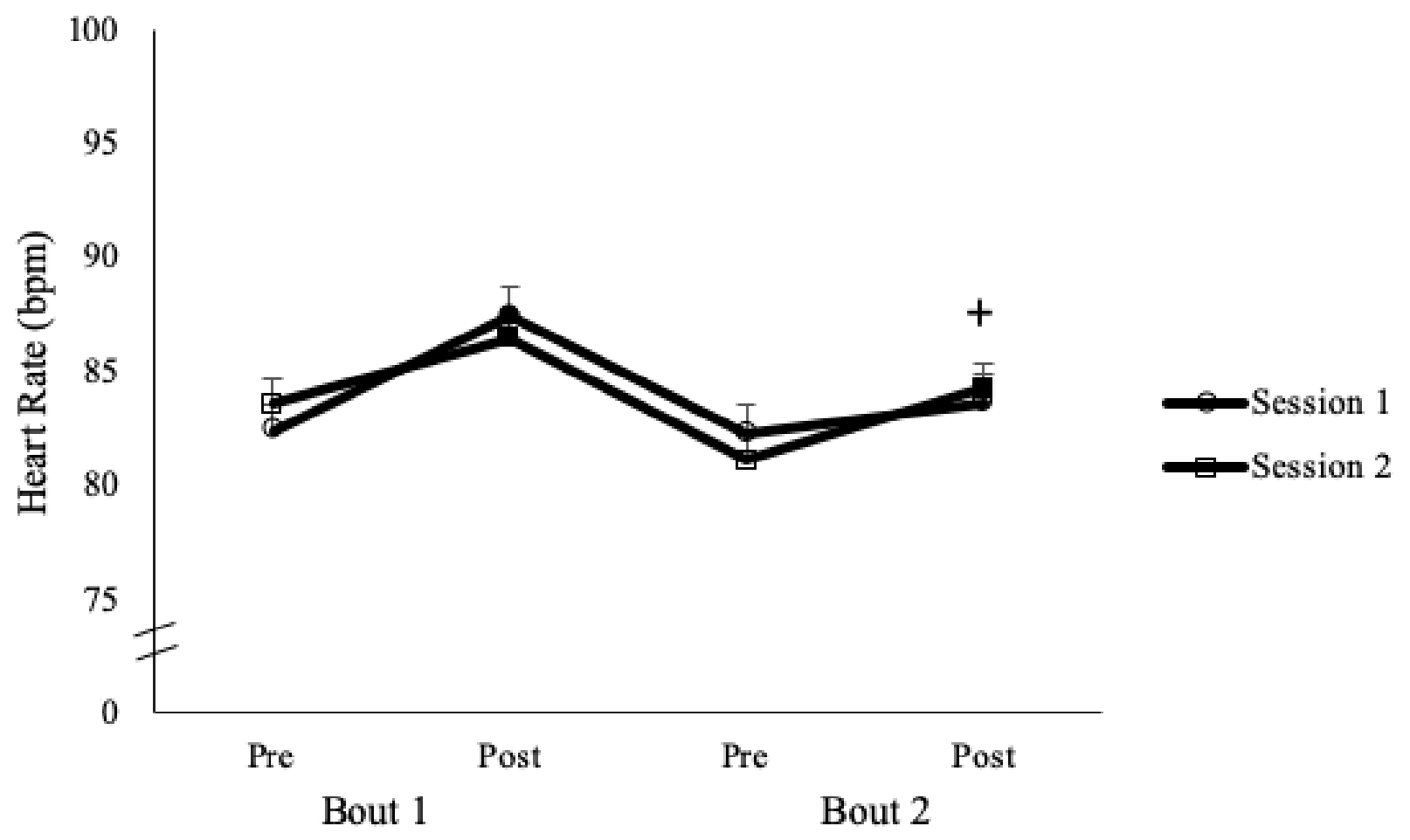

Figure 15. Mean (+/- SEMs) ratings are shown for heart rate for order $\mathrm{x}$ bout $\mathrm{x}$ time effects as a function of flavor (fruit vs. tobacco) x bout (1 vs. 2) x time (pre vs. post) for session 1 and session 2 . Filled symbols $=$ significant differences pre- to post- within bout $++=$ significant differences between sessions. 
Appendix A

Telephone Screening Questionnaire

Date:

Interviewer:

Interviewer: "I would like to ask you some questions about yourself and your health status as well as your use of tobacco, alcohol, and other drugs. The purpose of these questions is to determine whether or not you are eligible to participate in the study that I just described to you. All of your responses are confidential. You are not required to answer any question and you may stop this interview at any time. May I begin the questions?"

Document caller's response by circling either: Yes or No

If Yes: begin form. If No: thank the caller and stop the interview.

How did you hear about us/our study?

Personal Information:

1. "What is your first name?"

2. "What is a phone number at which you can be contacted?"

3. "If we call and you are not available, may we leave a message?" Yes or No

4. "What is your date of birth?"

General health status:

1. "Are you currently under a doctor's care for a medical condition?" Yes or No

If Yes: "Please describe the concern or problem":

2. "Do you have any chronic health concerns or problems?" Yes or No

If Yes: "Please describe the condition":

3. "Are you taking any prescription or over-the-counter medications?" Yes or No

If Yes: "Please identify the medication":

4. Do you have any psychiatric conditions like depression or anxiety? Yes or No

If Yes: "Please describe the condition":

5. "Have you ever been diagnosed with high or low blood pressure?" Yes or No

For women only:

If Yes: "Please indicate whether it is high or low":

6. "Are you currently pregnant?"

7. "Are you currently breast-feeding a child?"

Yes or No

Yes or No

\section{Tobacco Use:}

1. "Do you currently smoke tobacco cigarettes?"

2. "Have you ever smoked a tobacco cigarette?"

Yes or No

a) If yes, how many lifetime uses?

\section{Electronic Cigarette Use:}


1. "Do you currently use an electronic cigarette?"

2. "Have you ever used an electronic cigarette in your lifetime, even one puff??"

a) If yes, about how many times have you used one in your lifetime?

Yes or No

Yes or No

3. "Do you remember what nicotine concentration of liquid you used?"

4. "Do you remember what flavor of liquid you used?"

5. "Do you remember what brand of liquid you used?"

$(\mathrm{mg} / \mathrm{ml})$

Other Tobacco Use:

1. "Have you used any of the following other nicotine/tobacco products?"

\begin{tabular}{|c|c|c|c|}
\hline & EVER & EVER & $\begin{array}{c}\text { If 'yes', estimate how many days } \\
\text { you have used this product in the } \\
\text { past month, if any? }\end{array}$ \\
\hline Large cigars & No & Yes & \\
\hline $\begin{array}{c}\text { Cigarillos (e.g., Black \& Milds) } \\
\text { or small cigars }\end{array}$ & No & Yes & \\
\hline $\begin{array}{c}\text { Waterpipe (a.k.a. hookah or } \\
\text { shisha) }\end{array}$ & No & Yes & \\
\hline $\begin{array}{c}\text { Smokeless tobacco } \\
\text { (snuff/dip/chew/snus) }\end{array}$ & No & Yes & \\
\hline $\begin{array}{c}\text { Electronic Cigarette (if cigarette } \\
\text { smoker) }\end{array}$ & No & Yes & \\
\hline Other: __nes & No & Yes & \\
\hline
\end{tabular}

\section{Illicit Drug Use:}

1. "Have you used any of the following other drugs for recreational purposes in the past month?"

\begin{tabular}{|c|l|l|l|}
\hline & & & $\begin{array}{c}\text { If 'yes', estimate how } \\
\text { many days you have used } \\
\text { this product in the past }\end{array}$ \\
\hline Alcohol & No & Yes & \\
\hline Marijuana / Spice / K2 & No & Yes & \\
\hline $\begin{array}{c}\text { Stimulants (e.g., cocaine, amphetamine, } \\
\text { etc.) }\end{array}$ & No & Yes & \\
\hline \begin{tabular}{l} 
Opiates (e.g., heroin, oxycodone, etc.) \\
\hline
\end{tabular} & No & Yes & \\
\hline
\end{tabular}




\begin{tabular}{|l|l|l|l|}
\hline Other: & No & Yes & \\
\hline
\end{tabular}

Interviewer: "Thank you for responding to these questions. I need to pass on your responses to the principal investigator who will then determine whether or not you are eligible to participate in a study. If you are eligible, someone will contact you within approximately one week. If you are not eligible for this study, then you will not be contacted."

[If respondent does not have a phone, they can call us back in a few days] 


\section{Appendix B}

Demographic Information

Participant ID:

Today's Date:

Age

Years:

Date of birth:

Ethnicity

o Hispanic or Latino o Not Hispanic or Latino

Race

o American Indian/Alaskan Native o Asian/Native Hawaiian or other Pacific Islander
o Black or African American
o White
o Other/Unknown

\section{Gender}
o Male
o Female

\section{Marital status}
o Single
o Married
o Separated
o Divorced
o Widowed

\section{Education}

Years: $($ For example, High school $=12$, College degree $=16$, etc. $)$

\section{Primary employment}

o Unemployed o Part Time (0-30 hrs/wk) o Full Time (>30 hrs/wk) o Student 


\section{Appendix C \\ Medical History and Drug Use Form}

Participant ID:

Date:

\section{General health status:}

Are you under a doctor's care for a medical condition? below) (If yes, please describe

Are you taking any prescription medications? (If yes, please identify below)

Do you have any chronic health concerns or problems? (If yes, please describe below)

Do you have any psychiatric conditions? (If yes, please describe below)

\section{For women only:}

Are you currently pregnant? (yes or no)

Are you currently breast-feeding a child? (yes or no)

\section{Cigarette Use:}

Have you ever smoked tobacco cigarettes? ( if yes, number of lifetime uses)

Do you currently smoke tobacco cigarettes? (yes or no) if no, skip to the next section

\section{ECIG Use:}

Do you currently use an ECIG? (yes or no)

Have you ever used an ECIG, even one puff? (yes or no) if no skip to next section

a) About how many times have you used an ECIG in your lifetime?

b) Do you remember what nicotine level you used? $(\mathrm{mg} / \mathrm{ml}$ or $\%)$

c) Do you remember what flavor of ECIG you used?

\section{Other Tobacco Use:}

Have you ever used any other nicotine/tobacco products? (yes or no)

Circle all products below that you have ever used, for each item used please indicate \# of lifetime uses: 
Cigars / cigarillos / small cigars (\#uses)

Smokeless tobacco (snuff, dip, chew) / snus (\#uses)

Hookah / waterpipe (\#uses)

Electronic cigarette (\#uses)

Nicotine gum / patch / lozenge / inhaler (\#uses)

Other:

Do you currently use any other nicotine/tobacco products? (yes or no)

Circle all products below that you have used in the past 30 days:

Cigars / cigarillos / small cigars Smokeless tobacco (snuff, dip, chew) / snus

Hookah / waterpipe Electronic cigarette

Nicotine gum / patch / lozenge / inhaler Other:

\section{Alcohol Use:}

Have you used alcohol in the past month? (yes or no) if no, skip to the next section

How many days out of the last 30 have you used alcohol? (number of days)

Have you ever been treated for alcohol abuse/dependence? (yes or no)

\section{Other Drug Use:}

Have you used any illegal drugs within the past month? (yes or no)

If yes, please identify which drugs: 


\section{Appendix D \\ Electronic Cigarette Outcome Expectancy Short Scale}

The following scale includes statements about outcomes that might happen to you if you used ecigarettes. Please rate how LIKELY or UNLIKELY you believe each outcome would be for you if you used e-cigarettes. For example, if you believe that e-cigarette use would definitely make you "feel good," circle 9. If you believe that e-cigarette use would never make you "feel good," circle 0 . And if you believe e-cigarette use would only slightly decrease or increase your chance offeeling good, circle 4 or 5.

What is the likelihood that the following outcomes would happen to you if you used e-cigarettes? (Response Options: 0:Unlikely,..., 9:Likely)

Become more popular

$\begin{array}{llllllllll}0 & 1 & 2 & 3 & 4 & 5 & 6 & 7 & 8 & 9\end{array}$

Feel relaxed

$\begin{array}{llllllllll}0 & 1 & 2 & 3 & 4 & 5 & 6 & 7 & 8 & 9\end{array}$

Hurt lungs

$\begin{array}{lllllllllll}0 & 1 & 2 & 3 & 4 & 5 & 6 & 7 & 8 & 9\end{array}$

Enjoy "smoking" without bothering others

$\begin{array}{lllllllllll}0 & 1 & 2 & 3 & 4 & 5 & 6 & 7 & 8 & 9\end{array}$

Look awkward

$\begin{array}{llllllllll}0 & 1 & 2 & 3 & 4 & 5 & 6 & 7 & 8 & 9\end{array}$

Become addicted to e-cigarettes

$\begin{array}{lllllllllll}0 & 1 & 2 & 3 & 4 & 5 & 6 & 7 & 8 & 9\end{array}$

Feel bad taste

$\begin{array}{llllllllll}0 & 1 & 2 & 3 & 4 & 5 & 6 & 7 & 8 & 9\end{array}$

Smell good

$\begin{array}{llllllllll}0 & 1 & 2 & 3 & 4 & 5 & 6 & 7 & 8 & 9\end{array}$




\section{Appendix E}

ECIG Risk Perception

Please indicate the degree to which you agree or disagree with the following statements regarding electronic cigarettes.

Electronic cigarettes are less harmful than regular cigarettes.
o Strongly disagree
o Disagree
o Agree
o Strongly Agree

Electronic cigarettes can help people quit smoking.
o Strongly disagree
o Disagree
o Agree
o Strongly Agree

Electronic cigarettes are less addictive than cigarettes.
o Strongly disagree
o Disagree
o Agree
o Strongly Agree 


\section{Appendix F \\ Exit Interview \\ ECIG Use History: For the next 9 questions, I'd like for you to think about the times in your life when you used an ECIG.}

1. Did the ECIGs that you used contain nicotine never, sometimes or always?

2. Do you remember the amount(s) of nicotine that those ECIGs contained? [Participants can provide more than one nicotine concentration level if they believe that those previous devices had different concentrations]

3. Do you remember what flavor(s) of liquid you used in those ECIGs? List all flavors used/tried.

4. Of those flavors that you used, did you have any that you liked the most?

5. Of those flavors that you used, did you have any that you disliked?

6. Do you remember what type and/or brand of ECIG device you used? Please list all that apply and provide as many details as possible.

7. Where did you obtain the ECIG(s) you used? For example, did you get it from a friend, a family member, or a stranger? Did you get any free samples or purchase an ECIG? List all that apply.

8. Do you remember where you were / what you were doing when you used the ECIG(s)? For example, were you at home, hanging out with friends, at a party/bar? List all that apply. 
9. What were some reasons why you decided to use an ECIG? [Here you can prompt them to give reasons for the first time specifically, and then any of the other times]

\section{Study Experience: For the next 4 questions, we'd like to ask you to share your feelings about the experience of participating in this study.}

1. I'd like for you to think about the ECIG device and liquid that you used in this study. We are interested in your thoughts about the experience with that particular device and liquid. [Here we want to make sure to touch upon the following factors: a) nicotine concentration, b) flavor, c) device type, d) amount of ECIG liquid consumed within a session]

2. For what reason(s) did you decide to take part in this study?

3. What aspects of the study did you like or dislike in particular?

4. Looking back, was the study what you expected or not? In what way was the study different from what you expected? How do you feel about that? 


\section{Appendix G}

Direct Effects of Nicotine Scale

These phrases may or may not describe how you feel right now. Please respond to each word or phrase with how you feel RIGHT NOW by drawing a vertical mark anywhere along the horizontal line.

\section{Not at all}

1. Nauseous

2. Dizzy

3. Lightheaded

4. Nervous

5. Sweaty

6. Headache

7. Excessive salivation

8. Heart pounding

9. Confused

10. Weak
Extremely
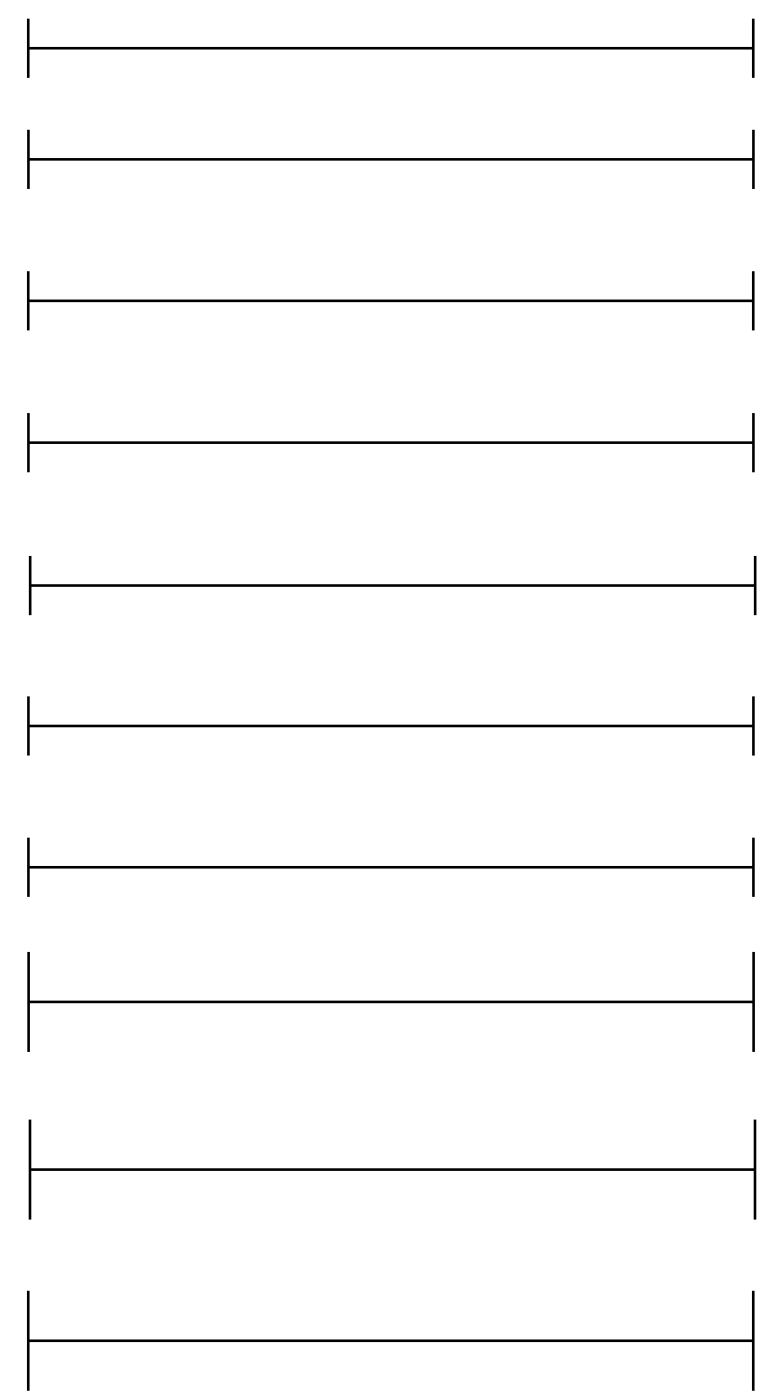


\section{Appendix $\mathrm{H}$}

Direct Effects of ECIG USE

These phrases may or may not describe how you feel right now. Please respond to each word or phrase with how you feel RIGHT NOW by drawing a vertical mark anywhere along the horizontal line.

1. Was the product satisfying?

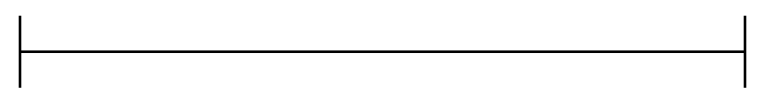

2. Was the product pleasant?

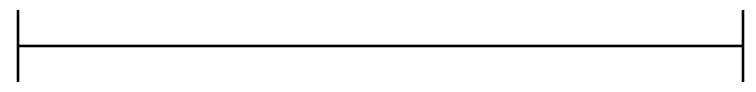

3. Did the product taste good?

4. Did the product make you dizzy?

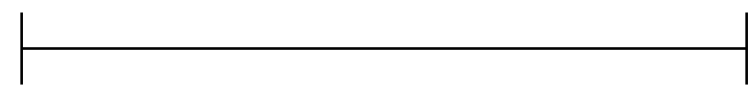

5. Did the product calm you down?
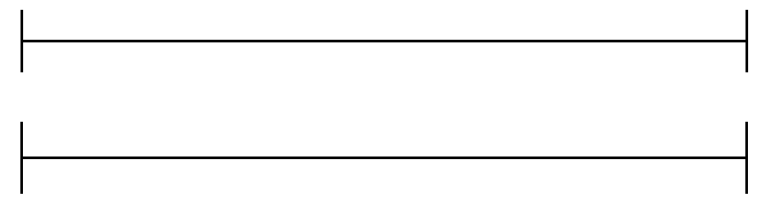

6. Did the product help you concentrate?

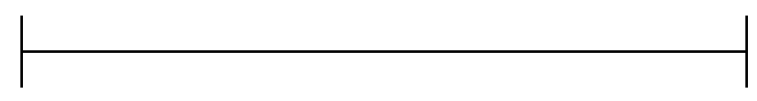

7. Did the product make you feel more awake?

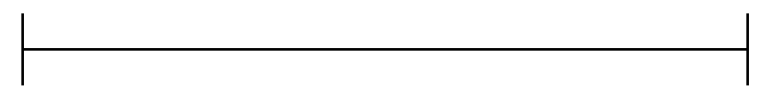

8. Did the product reduce your hunger for food?

9. Did the product make you sick?

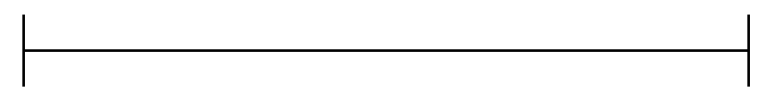


Appendix I

For each item, please indicate how you would describe the ECIG you just used by placing a mark on the vertical numbered line.

\section{How would you describe the overall flavor sensation of the ECIG you just used?}

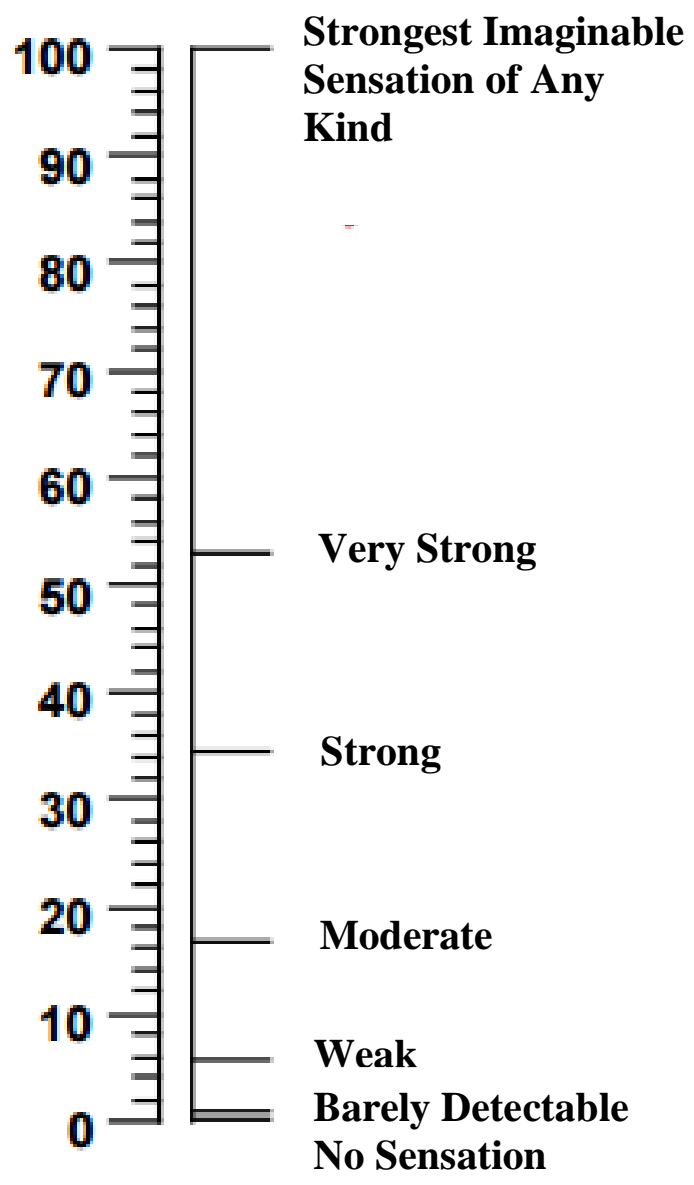


How would you describe the overall harshness/irritancy of the ECIG you just used?

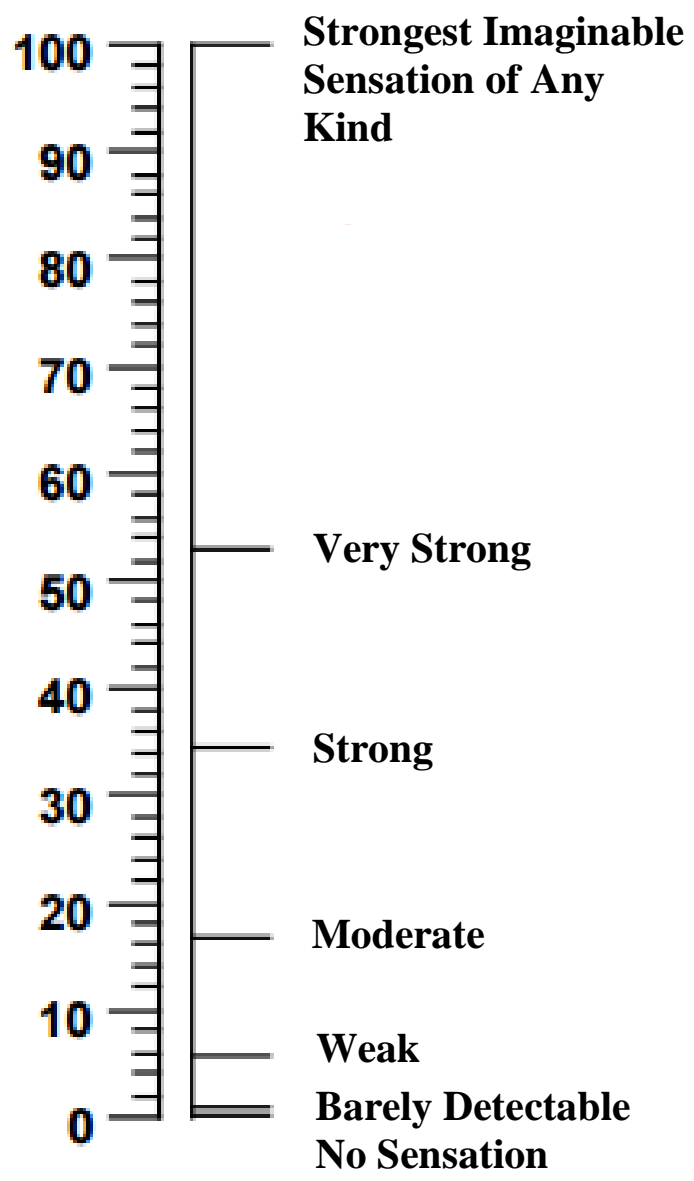


How would you describe the throat hit of the ECIG

$$
\text { you just used? }
$$

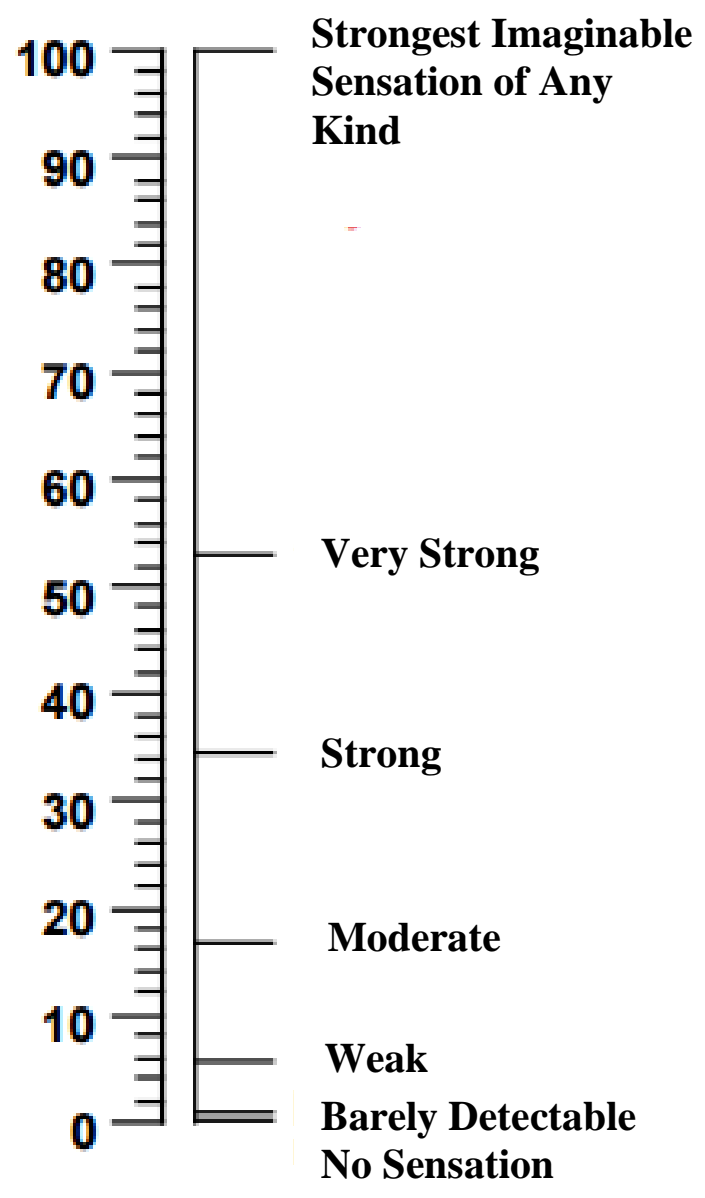




\section{Appendix $\mathbf{J}$}

End-of-Study Flavor Choice (Mango)

Date:

Participant ID:

In this study, you used two different flavors of JUUL. During one visit, you used a JUUL that was flavored with tobacco. During another visit, you used a JUUL that was flavored with mango. For each flavor option below, circle which visit you think you used that flavor that is listed

\begin{tabular}{|l|c|c|c|c|}
\hline \multicolumn{2}{|c|}{ I used TOBACCO in } & & \multicolumn{2}{c|}{ I used MANGO in } \\
\hline Visit 1 & Visit 2 & & Visit 1 & Visit 2 \\
\hline
\end{tabular}


End-of-Study Flavor Choice (Fruit Medley)

Date:

Participant ID:

In this study, you used two different flavors of JUUL. During one visit, you used a JUUL that was flavored with tobacco. During another visit, you used a JUUL that was flavored with fruit medley. For each flavor option below, circle which visit you think you used that flavor that is listed

\begin{tabular}{|l|c|c|c|c|}
\hline \multicolumn{2}{|c|}{ I used TOBACCO in } & & \multicolumn{2}{c|}{ I used FRUIT MEDLEY in } \\
\hline Visit 1 & Visit 2 & & Visit 1 & Visit 2 \\
\hline
\end{tabular}

